Portland State University

PDXScholar

Dissertations and Theses

Dissertations and Theses

7-5-2018

Electro-Drop Bouncing in Low-Gravity

Erin Stivers Schmidt

Portland State University

Follow this and additional works at: https://pdxscholar.library.pdx.edu/open_access_etds

Part of the Aerodynamics and Fluid Mechanics Commons, and the Fluid Dynamics Commons Let us know how access to this document benefits you.

Recommended Citation

Schmidt, Erin Stivers, "Electro-Drop Bouncing in Low-Gravity" (2018). Dissertations and Theses. Paper 4441.

https://doi.org/10.15760/etd.6325

This Thesis is brought to you for free and open access. It has been accepted for inclusion in Dissertations and Theses by an authorized administrator of PDXScholar. Please contact us if we can make this document more accessible: pdxscholar@pdx.edu. 


\title{
Electro-Drop Bouncing in Low-Gravity
}

\author{
by
}

Erin Stivers Schmidt

A thesis submitted in partial fulfillment of the requirements for the degree of

Master of Science

in

Mechanical Engineering

Thesis Committee:

Mark Weislogel, Chair

Raúl Bayoán Cal

Gerald Recktenwald

Portland State University

2018 


\section{Abstract}

We investigate the dynamics of spontaneous jumps of water drops from electrically charged superhydrophobic dielectric substrates during a sudden step reduction in gravity level. In the brief free-fall environment of a drop tower, with a non-homogeneous external electric field arising due to dielectric surface charges (with surface potentials $0.4-1.8 \mathrm{kV}$ ), body forces acting on the jumped drops are primarily supplied by polarization stress and Coulombic attraction instead of gravity. This electric body force leads to a drop bouncing behavior similar to well-known phenomena in 1- $g_{0}$, though occurring for much larger drops $(\sim 0.5 \mathrm{~mL})$. We show a simple model for the phenomenon, its scaling, and asymptotic estimates for drop time of flight in two regimes: at short-times close to the substrate when drop inertia balances Coulombic force due to net free charge and image charges in the dielectric substrate and at long-times far from the substrate when drop inertia balances free charge Coulombic force and drag. The drop trajectories are controlled primarily by the dimensionless electrostatic Euler number $\mathbb{E} u$, which is a ratio of inertial to electrostatic forces. To experimentally determine values of $\mathbb{E} u$ we conduct a series of drop tower experiments where we observe the effects of drop volume, net free charge, and static surface potential of the superhydrophobic substrate on drop trajectories. We use a direct search optimization to obtain a Maximum Likelihood Estimate for drop net charge, as we do not measure it directly in experiment. For $\phi \mathbb{E} u / 8 \pi>1$ drops escape the electric field, where $\phi$ is a drop to substrate aspect ratio. However, we do not observe any escapes in our dataset. With an eye towards engineering applications 
we consider the results in light of the so-called low-gravity phase separation problem with a worked example. 
To Mom, Laura, and the DDT team 


\section{Acknowledgements}

This work was supported by NASA Cooperative Agreement NNX12A047A. I am also greatly indebted to Andrew Greenberg for his frequent and sage technical advice, and to Joe Shields for his assistance with drop tower experiments. 


\section{Contents}

Abstract

Acknowledgements iv

$\begin{array}{lll}1 & \text { Introduction } & 1\end{array}$

1.1 Spontaneous Drop Jump . . . . . . . . . . . . . . . . . . . 5

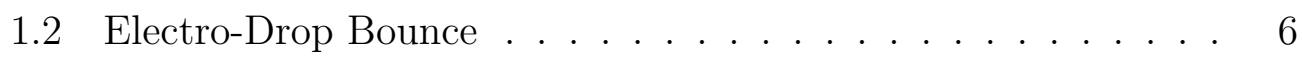

1.3 Applications . . . . . . . . . . . . . . . . . . . . 11

1.4 Overview of this Thesis . . . . . . . . . . . . . . . . . 13

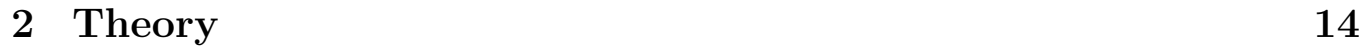

2.1 Equation of Motion . . . . . . . . . . . . . . . . . . . . 14

2.2 The Electric Field . . . . . . . . . . . . . . . . . . . . . . . 20

2.3 Scaling . . . . . . . . . . . . . . . . . . . . . . . . . . . 23

$2.3 .1 \quad$ Inertial Electro-Image Limit . . . . . . . . . . . . . 24

2.3 .2 Inertial Electro-Viscous Limit . . . . . . . . . . . . . . 25

2.4 Asymptotic Estimates . . . . . . . . . . . . . . . . . 26

$2.4 .1 \quad$ Inertial Electro-Image Limit . . . . . . . . . . . . . 26

2.4 .2 Inertial Electro-Viscous Limit . . . . . . . . . . . . . . 28

2.5 Summary $\ldots \ldots \ldots \ldots \ldots$ 
3 Methodology 33

3.1 Overview . . . . . . . . . . . . . . . . . . . . 33

3.2 Experimental Methods . . . . . . . . . . . . . . . . . . . . 34

3.3 Data Reduction . . . . . . . . . . . . . . . . . . . . . . . 43

3.4 Parameter Estimation . . . . . . . . . . . . . . . . 43

$3.4 .1 \quad$ Inverse Problems . . . . . . . . . . . . . . . . . 45

3.4 .2 Smoothing . . . . . . . . . . . . . . . . . . . . 48

$3.4 .3 \quad$ Optimization . . . . . . . . . . . . . . . . 53

3.4 .4 Identifiability . . . . . . . . . . . . . 56

3.5 Summary . . . . . . . . . . . . . . . . . . . . 62

\begin{tabular}{|ll|l}
\hline 4 & Results & 63
\end{tabular}

4.1 Parameter Estimates . . . . . . . . . . . . . . . . . 63

4.2 Model Validation . . . . . . . . . . . . . . . . . . . 66

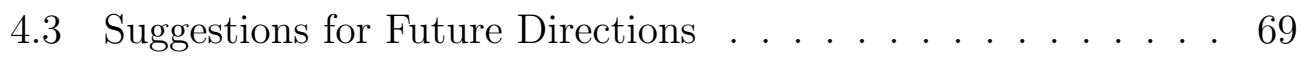

4.4 Conclusion . . . . . . . . . . . . . . . . . . . . . . . 73

\section{Appendices}

\begin{tabular}{ll}
\hline A Parallel Plate Method & 78
\end{tabular}

\begin{tabular}{|r|r|}
\hline B Charge Mitigation & 81
\end{tabular} 


\section{List of Figures}

1.1 The trajectory of a $0.2 \mathrm{~mL}$ spontaneous drop jump is captured in a composite image over a period $\sim 0.67$ s presented at $\approx 10$

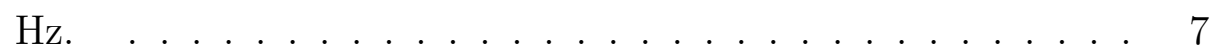

1.2 The trajectory of a $0.5 \mathrm{~mL}$ drop is captured in a composite image over a single bounce period $(\sim 1.25 \mathrm{~s})$. The surface potential of the superhydrophobic dielectric in this example is $\varphi_{s}=1.25 \pm 0.41 \mathrm{kV} . \ldots \ldots \ldots \ldots$

2.1 Schematic representation of drop jump with return and rebound from an electrically charged superhydrophobic substrate.

The characteristic time and length scales $t_{c}$ and $y_{c}$ describe the time of flight and apoapse associated with the drop trajectory. 15

$2.2 \quad$ A log-log plot of the magnitude of the dimensionless electric field $E$. . . . . . . . . . . . . . . . . . . . . . . . . . . . . . . . . . 22

2.3 Short-time scaled drop trajectories for various values of $\mathbb{E} u$, Ig. The trajectory reduces to the classical $\mathcal{O}(1)$ solution for small values of Ig. It should be noted that despite what is implied by these plots $\mathbb{I g}$ is not necessarily independent of $\mathbb{E} u$, as they share $q$, and $E_{0}$ as common factors, $U_{0} \propto R_{d}^{2}$, and $m \propto R_{d}^{3}$. . . 27 
$2.4 \quad$ Long-time scaled drop trajectories for various values of $\phi \mathbb{E} u . \quad 29$

$2.5 \mathcal{O}\left(\phi^{3} \mathbb{E u}^{3}\right)$ accurate asymptotic estimate of drop time-of-flight

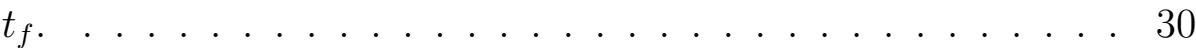

3.1 Portion of drop at the initial condition, resting on a superhydrophobic surface in the Cassie-Baxter state just prior to release of the experiment into free-fall. The variables $q, \sigma$, and $V_{d}$ are the drop net free charge, dielectric surface charge density, and drop volume, respectively. . . . . . . . . . . . . 34

3.2 (a) The electro-drop bounce experiment hardware mounted on a drop tower rig. (b) Close up of the experimental test cell. . . 38

3.3 The Furmidge model of the hysteresis of the contact angle plotted as a function of static contact angle $\theta$ and roll-off angle $\alpha$ for $1 \mathrm{~mL}$ drops. The measured static contact angles and roll-off angles of several superhydrophobic surfaces including the laser-etched PMMA used in this work are also shown annotated with the characteristic roughness lengthscale $R_{q}$ of the surface. . . . . . . . . . . . . . . . 39

3.4 SEM image of the superhydrophobic surface. . . . . . . . . . . 40

3.5 Charge decay in the dielectric laminates for several numbers of layers. . . . . . . . . . . . . . . . . . . . . . 4 42 
$3.6 \quad$ (a) Schematic noisy drop trajectory data. We see that 1D Gaussian convolution and Wiener filters suffer from significant end effects. At this scale Butterworth and Savistsky-Golay filters are nearly indistinguishable. (b) Comparing Butter-

worth and Savitsky-Golay filtered first derivatives of the above schematic trajectory we see that the Butterworth filter also suffers from a slight end effect. This implies that the optimization will find a different optima of the likelihood depending on which type of filter is used. . . . . . . . . . . . . . . . 50

3.7 Signal power spectra with peak at $1 \mathrm{~Hz}$, which is the drop trajectory parabola itself, and smaller peaks in the $\mathrm{kHz}$ range corresponding to various noise frequencies. The Savitsky-Golay and Butterworth filters appear to have the least distortion and attenuate the noise at the $2 \mathrm{kHz}$ peak. . . . . . . . . . 51

3.8 Convergence history of MLE optimization by the Nelder-Mead algorithm for a typical drop trajectory. . . . . . . . . . . 57

3.9 A series of filtered drop trajectories arranged by increasing apoapse. The blue dots represent either the beginning and end of the experiment, or points at which the drop is either coming into or leaving contact with the surface. . . . . . . . . 58 
3.10 A set of drop trajectories showing the results of the parameter estimation. The trajectories are shown only up to the apoapse of the first bounce. The $(-)$ lines show the ODE solution with the given MLE parameter vector. $\chi^{2}$ goodnessof-fit varies between $1 \times 10^{-5}$ and $1 \times 10^{-8}$ with the better fit occurring typically for the drops with the lowest apoapses. . . 59

4.1 Scatter plot matrix of main parameters $E_{0}, U_{0}, V_{d}$, and q. . . . 64

4.2 Charge $q$, as a function of the measured variables $V_{d}, \varphi_{s}$. . . 65

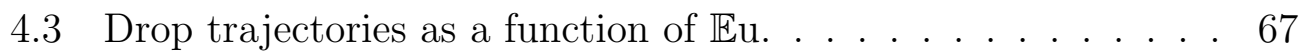

4.4 Non-dimensional trajectories with the short-time scaling. . . . 68

4.5 Dimensional time-of-flight asymptotic estimates $t_{c} t_{f}$ compared with experimental time-of-flight $t_{b}$. . . . . . . . . . . . 69

4.6 Simulated forces acting on the drop up to the first apoapse. . . 70

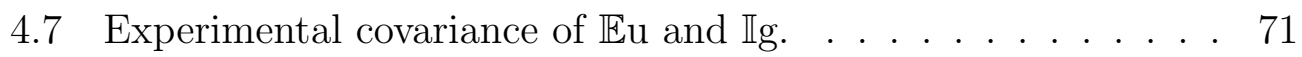

4.8 Dimensionless contact time $t_{j} / \tau$ compared with impact $\mathbb{W e}$ and $\mathbb{B o}_{e} . \ldots \ldots \ldots \ldots \ldots \ldots$

4.9 Impact coefficient of restitution $C_{r}$ compared with impact $\mathbb{W e}$ and $\mathbb{B B o}_{e} \ldots \ldots \ldots \ldots \ldots \ldots \ldots \ldots$ 


\section{Chapter 1}

\section{Introduction}

"When the influence of gravity on fluid behavior is diminished or removed, other forces, otherwise of small significance, can assume paramount roles." - NRC Report to NASA, 2003 [1]

Our terrestrially born intuition about how liquids flow is easily confounded in a low-gravity environment. This should come as little surprise, as we are creatures evolved at the bottom of a gravity well; gravity is natural to us. However when the magnitude of gravitational body forces become small, other forces come into play in fluid dynamical problems, which are otherwise negligible under normal circumstances in $1-g_{0}$, where $g_{0}=9.81$ $\mathrm{m} / \mathrm{s}^{2}$ is the standard gravitational acceleration at sea-level. Our $1-g_{0}$ cognitive bias then leads to a plethora of problems which are both simultaneously trivially easy to solve in $1-g_{0}$, and which despite decades of study continue to elude solutions in a low-gravity context. One example of these problems is the so-called "phase separation" problem of separating a gas phase from a multi-phase flow (or the reverse case), without the aid of buoyancy due to gravity. Sans buoyancy, otherwise mundane activities such as venting a gas or settling a liquid in a tank become problematic [2]. Trapped bubbles 
can vapor lock life-support, power, or propulsion systems [3]. Issues of phase separation have so bedeviled human endeavors in space that an entire Apollo Saturn 1B mission (AS-203) was earmarked to study them [4]. This problem has for some time motivated a quest for substitute body forces, and the present work follows in that well-worn tradition.

A solid beginning to rectifying our problem of poor low-gravity intuition, would be to quantify how wrong our intuition is. First, let us clear up a common misnomer about the state of "zero-gravity". The theory of general relativity is based on a fundamental equivalence of gravity and acceleration. By measuring acceleration alone, one cannot determine whether one is in gravity-free space or free-falling in a gravitational field (or conversely whether or not one is uniformly accelerating, or standing at rest in a gravitational field). This is sometimes called the equivalence principle of general relativity (e.g. the equivalence of gravitational and inertial mass). In theoretical terms, a 'zero-g' experiment performed in a drop tower on the surface of the Earth is equivalent to the same experiment performed in the nearly gravity-free deep space between galaxies, despite the fact that the drop tower itself is enveloped in a $1-g_{0}$ gravity field. At the orbital altitude of the International Space Station (ISS) the gravity level is still nearly $90 \%$ of its value at sea-level on Earth, but the astronauts aboard ISS feel weightlessness because they are in a state of free-fall. In a practical sense true zero-g does not exist anywhere in the universe as there are always small, but nonetheless, measurable forces which can accelerate any massive body. For instance, in low-Earth orbit accelerations due to aerodynamic resistance in the tenuous outer atmosphere are on the order of $1 \times 10^{-6} g_{0}$ (from hence comes the term "microgravity"). 
This small drag acceleration provides some ersatz gravity, but there are also other forces present. Foremost of these are capillary forces.

Capillary forces are due to the cumulative effects of a large number of very short range molecular interactions and in a $1-g_{0}$ context are usually quite weak, relatively speaking. However, in a low-gravity environment other forces are so weak that capillary forces become extraordinarily strong in a relative sense. In comparing the relative magnitudes of gravity and the capillary force we arrive at our figure of merit for assessing how wrong our fluid mechanical intuition is likely to be in a low-gravity context. This figure of merit is the Bond number dimensionless group

$$
\mathbb{B o} \equiv \frac{g R^{2} \Delta \rho}{\gamma}
$$

where $g$ is the acceleration, $R$ is the characteristic interfacial length scale, $\Delta \rho$ is the difference in densities across the interface (which simplifies to $\rho$, for large $\Delta \rho$, as in the case of an air-water interface), and where $\gamma$ is the surface tension. In cases where $\mathbb{B}$ o is small a liquid is effectively in lowgravity regardless of the nominal local gravity level. Very small water drops $(R<1 \mathrm{~mm})$, for instance, are in low-gravity for all intents and purposes and have nearly spherical shapes. Contrarily, for cases of large $R$ in a space environment, such as with large diameter spacecraft propellant tanks then $\mathbb{B}$ o 1 even though the acceleration remains small. This is actually a quite useful result, for on the basis of dimensional similarity it is possible to accurately simulate low-gravity fluid mechanics (say of large propellant tanks) even in small drop towers simply by scaling dimensionless groups like 
the Bond number.

As with capillary forces, electrostatic forces have a multitude of applications as substitutes for gravity. Electrohydrodynamic (EHD) body forces have been suggested to combat thermal stratification of propellants under low-g conditions where buoyancy induced natural convection is very small [5]. In this case electro-convection is an analog of natural convection due to a fluid temperature dependent dielectric permittivity gradient (as opposed to a fluid density gradient) in the presence of an electric body force field. The study also suggested applications in cryogenic tank vent screening and in reducing heat transfer to cryogenic propellants (and thus boiloff losses) by dielectrophoretically positioning ullage vapors around the tank walls as a thermal barrier. EHD forces have been studied for enhancing boiling heat transfer by promotion of bubble detachment and prevention of dryout, again as an ersatz buoyancy [6 8]. EHD heat pipes, which substitute an electrode structure for the capillary wicking structure of a conventional thermocapillary heat pipe can postpone the wicking limit [9]. These are restricted to the use of insulating dielectric liquids, which usually have relatively low thermal conductivity, but are highly robust against dryout, support active flow control, and have low viscous losses. Dielectrophoretic settling of cryogenic propellants, in both total and partial communication Propellant Management Device (PMD) configurations, has been studied analytically in both static [10] and dynamic cases [11], and in drop tower experiments [12]. EHD forces have also been applied to the low-g slosh baffling problem 13 15, and studied extensively for the mitigation of vapor pullthrough during tank draining and concomittent minimization of propellant residuals at burnout 
in a series of experiments at the NASA Lewis (now Glenn) Research Center $2.2 \mathrm{~s}$ drop tower facility [16].

\subsection{Spontaneous Drop Jump}

Low-gravity phase separation, drop dynamics, and the dynamics and applications of the so-called spontaneous drop or puddle jump, is an active area of study at the Portland State University Dryden Drop Tower laboratory (DDT). When a nonwetting, gravity-dominated sessile drop (e.g. a puddle), which is initially at rest on a surface in the Cassie-Baxter state suddenly undergoes a large step reduction in $\mathbb{B}$ o it will spontaneously jump away from the surface. The Cassie-Baxter state, sometimes also known as the Fakir state, is a metastable heterogenously non-wetting state on a textured surface characterized by the existence of a vapor-layer that separates roughness features of the surface from the bottom of the drop. The spontaneous drop jump was first observed experimentally in the Soviet Union by Kirko et al. [17] in 1970 for drops of mercury in hydrochloric acid, and later by Wollman et al. in 2016 for water drops in air in a set of experiments conducted using drop towers [18]. The kinetic energy of the jump is supplied by the defect in free surface energy as the new minimum energy surface equilibrium has approximately constant curvature. If the energy losses by viscous and contact line dynamics are neglected, the available kinetic energy is approximated by

$$
K E_{2}=S E_{2}-S E_{1}=\left[(\gamma A)_{g l}+(\gamma A)_{s g}\right]_{2}-\left[(\gamma A)_{l s}+(\gamma A)_{g l}+(\gamma A)_{s g}\right]_{1},
$$


where $K E$ and $S E$ are the respective kinetic and surface energies, $A$ is the surface area, the subscripts 1 and 2 denote the intial $1-g_{0}$, and low-g states, and the subscripts $g l, s g$, and $l s$ denote the gas-liquid, solid-gas and liquidsolid interfaces respectively. The motive force is due to the inertia of internal flows which occur as the interface deflects under the suddenly lessened $\mathbb{B}$. The characteristic time scale of the rolling up of the contact line scales as $t_{j} \sim\left(\rho V_{d} / \sigma\right)^{1 / 2}\left[19\right.$, which resembles the contact time, $\tau \approx 2.6\left(\rho R_{d}^{3} / \sigma\right)^{1 / 2}$ presented by Richard et al. in 2002 for the related problem of drops impacting hydrophobic surfaces in 1- $g_{0}[20]$. For drops with radial symmetry and sufficiently high initial $\mathbb{B}$ o, inward radial capillary waves coalesce at the axis, leading to geysering and creation of satellite droplets by the RayleighPlateau breakup of the geyser. In the case of smaller jumping drops the capillary waves are gradually damped by viscous forces. A time-lapsed composite photograph of spontaneous drop jump can be seen in Figure 1.1 .

The physics of these relatively massive drops (far beyond the $1-g_{0}$ millimetric capillary length scale) at once utterly defy terrestrial expectations about the ways in which liquid 'should' behave, and also are of critical practical importance to space systems design where examples of such large capillary length scale multiphase flows are commonplace.

\section{$1.2 \quad$ Electro-Drop Bounce}

During the 'rolling-up' of drops under ideal conditions, the spontaneous jump phenomenon is governed by a balance of inertia and surface tension forces, and once aloft the drop motion is nominally in a regime of pure drag. How- 


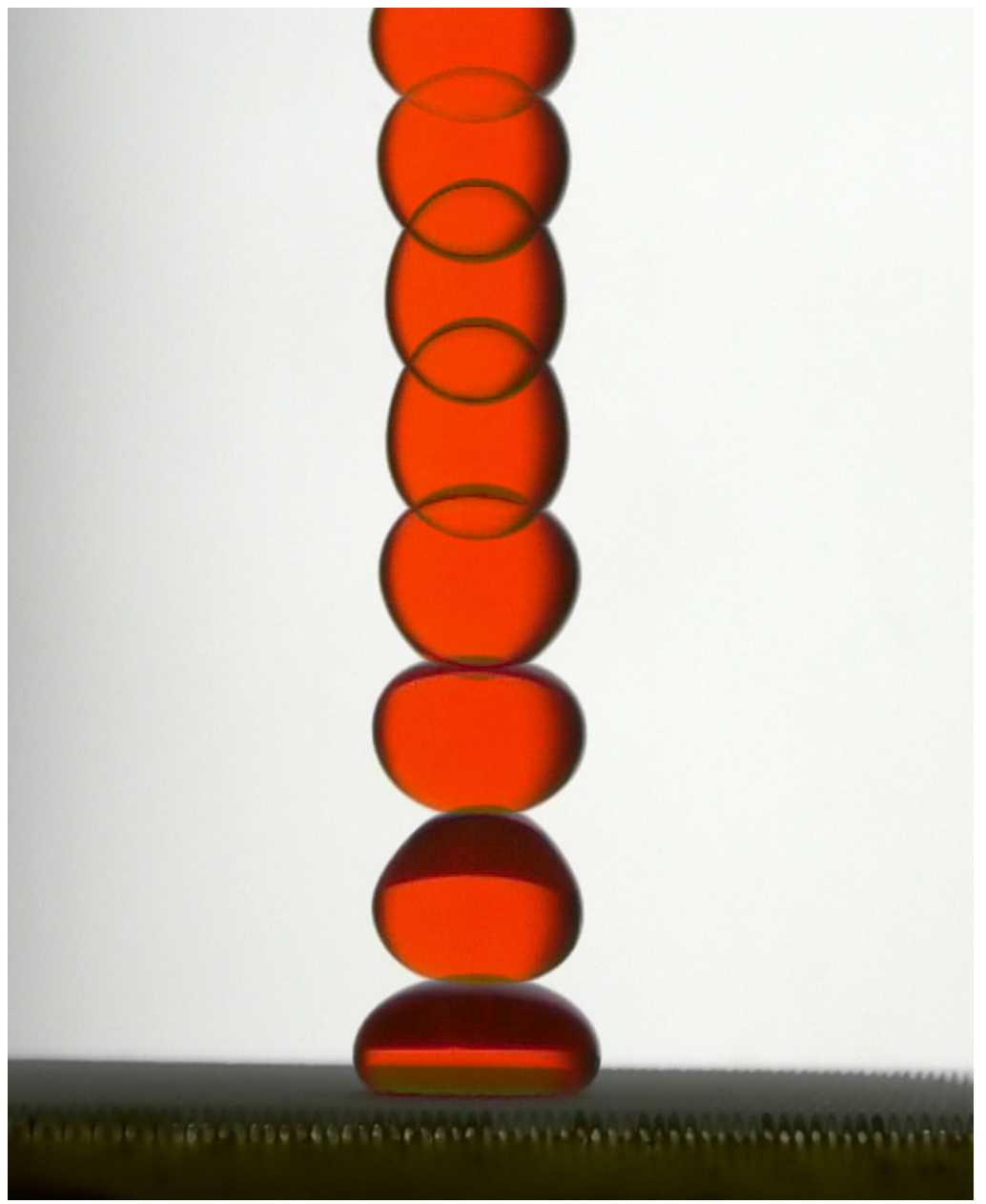

Figure 1.1: The trajectory of a $0.2 \mathrm{~mL}$ spontaneous drop jump is captured in a composite image over a period $\sim 0.67$ s presented at $\approx 10 \mathrm{~Hz}$.

ever, other forces can again come into play.

We have observed jumped drops to occasionally decelerate and return to the superhydrophobic surface, rebounding multiple times in the fashion of rigid bodies bouncing under $1-g_{0}$. The forces at work in such situations are presumably electrostatic in origin. Not without irony given our earlier discussion of underdeveloped low-gravity intuition, we note that though the electro-drop bounce was an accidental effect during our work on spontaneous 
drop jump, the phenomenon is interesting itself. This has motivated further study, and the 'electro-drop bounce' phenomenon is the subject of this thesis. A time-lapsed composite image showing an example of the phenomenon is shown in Figure 1.2

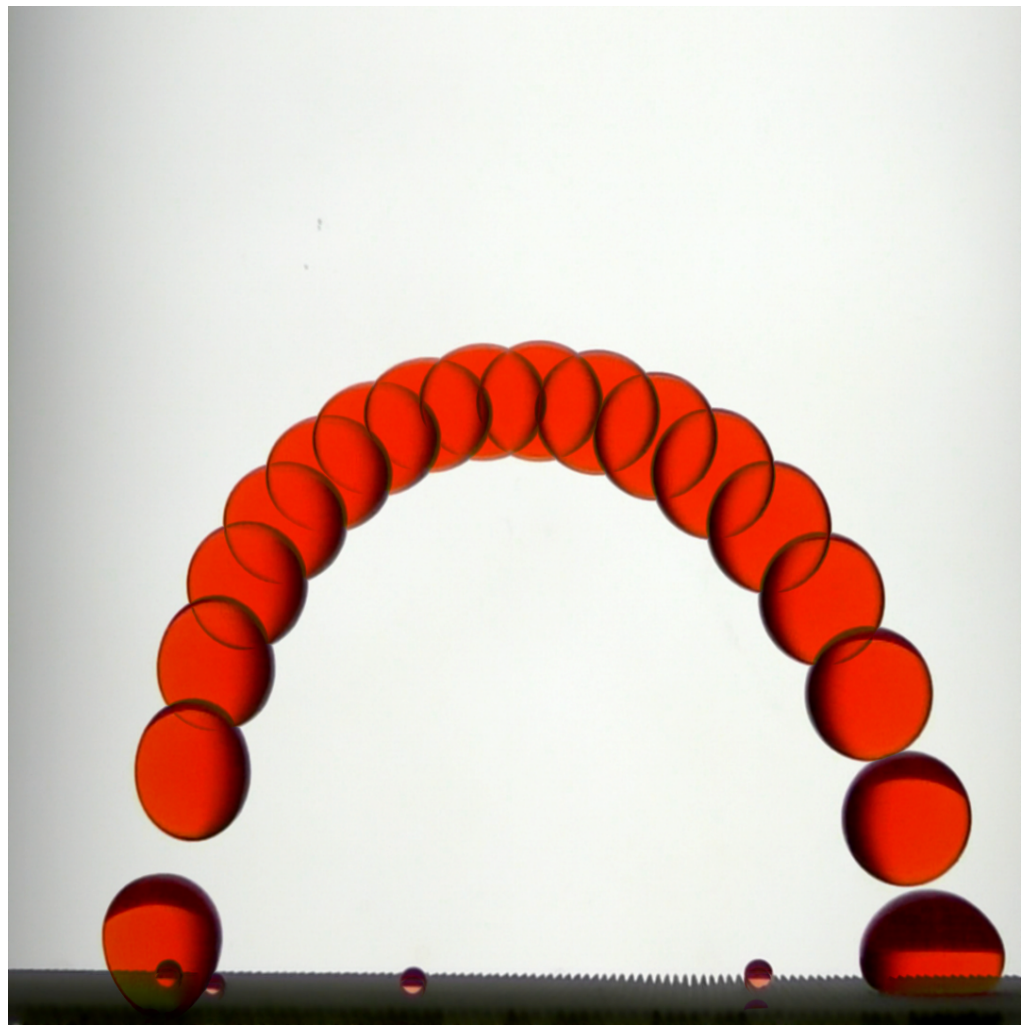

Figure 1.2: The trajectory of a $0.5 \mathrm{~mL}$ drop is captured in a composite image over a single bounce period $(\sim 1.25 \mathrm{~s})$. The surface potential of the superhydrophobic dielectric in this example is $\varphi_{s}=1.25 \pm 0.41 \mathrm{kV}$.

We begin with some preliminary observations of the phenomenon:

- Observed maximum drop (de-)accelerations are on the order of $\sim 30$ $\mathrm{cm} / \mathrm{s}^{2}$ for a range of drop volumes $0.03 \lesssim V_{d} \lesssim 0.5 \mathrm{~mL}$.

- The water drops are attracted to regions of high electric field. The horizontal (surface plane parallel) components of the drop trajectory 
usually oscillate about some central position during the experiment (except in cases of nearly pure 1-D vertical translation). For especially small drops close to the spontaneous drop jump limit $\left(V_{d} \sim 0.01 \mathrm{~mL}\right)$ the drops do not jump, but translate across the surface in a rolling regime until either they reach a local maximum of the electric field, or until their motion is sufficiently damped by contact line hysteresis where pinning ultimately arrests their motion.

- The drops appear to have net free charge. In cases of multiple simultaneous drop jumps the drops repel each other as they bounce or roll in orbital motion around regions of high field.

- The magnitude of the drop trajectory maxima (apoapses) appear to be related to the drop volume (mass), and initial jump velocity (inertia), and to the electric field strength.

There are several possible origins of the electric charge that are ultimately responsible for this phenomenon. It is well known that water acquires positive free charge when in contact with certain polymers, especially polytetrafluoroethylene (PTFE), through a process called contact charging 21]. PTFE, on the other hand, tends to readily acquire negative charge by contact with water. The superhydrophobic surfaces used in the spontaneous drop jump experiments have thin (nanometric) PTFE coatings, and we observe that it is extremely easy to produce significant surface potentials $\varphi_{s} \sim 100-500 \mathrm{~V}$ by simply flowing streams of distilled water over them. A study of this water on PTFE contact charging phenomenon was conducted by Yatsuzuka et al. 22, who suggest that this process results from formation of an electrical double 
layer driven by selective adsorption of $\left(\mathrm{OH}^{-}\right)$ions at the polymer surface; other recent work strongly supports this hypothesis [23, 24]. Yatsuzuka et al. also found that the specific charge on the drops in contact with the surface depends on their both surface velocity and conductivity. The contact charging mechanism is the most likely accidental source of charge on the suphydrophobic surfaces used in our work. Given the large roughness, or the ratio of projected to actual surface area, of the superhydrophobic surfaces used in the experiment, and given that the drops are initially in a CassieBaxter state, a somewhat electrically resistive air layer is maintained that reduces grounding of the drops despite the large potential difference between them and the surface charges. If this electrostatic potential is greater than the release of surface energy under the sudden change in $\mathbb{B}$, in the form of the drop kinetic energy, we expect that the drop will return to an equilibrium state on the charged surface which minimizes the potential.

The source of the net free charge on the drops is another issue. The drop charge could also be due to the contact charging mechanism mentioned previously. For instance, in a 1996 paper, NASA flight engineer Don Pettit discusses the problem of low-gravity flow induced charging of liquids, resulting ultimately from contact charging phenomenon [25]. Another mechanism for the drop charge is field-induced charging. Field-induced charging occurs due to physical breakup of a conductor having a field-induced dipole (e.g., a physical separation of charge). In our work this might occur when a drop is deposited on the charged surface by a grounded syringe. The metal syringe needle tip, and the liquid in the syringe itself are essentially a ground connection which is broken when the syringe is suddenly removed in the presence 
of an external electric field. Field-induced charging is at work in the famous Kelvin thunderstorm, and is applied in inkjet, and electrospay technologies, where in each case the breakup is by the Rayleigh-Plateau instability. Notably, in Pettit's aforementioned discussion of contact charging of liquids in low-g, he remarks on accidental electrostatic 'hula-ing' of silicone oil drops when ejected from a syringe in the vicinity of a highly-charged polymer surface during an experiment conducted aboard STS-5 by mission specialist Joseph Allen [25]. Depending on the (highly-variable) electrical conductivity of the silicone oil, and the material of the syringe used in the experiment, the charge could arise just as easily by field-induction as by contact charging. Relatedly, in a series of informal and somewhat whimsical experiments Pettit himself electrostatically orbited small water drops around a triboelectrically charged PTFE knitting needle while aboard ISS during expedition 30/31 26]. Again, in this case, the drop charge is likely field-induced.

\subsection{Applications}

This study of the electro-drop bounce phenomenon may have results which can be extended to useful applications in a space environment. External surfaces of spacecraft tend to become charged with time due to the space environment itself. Surface charging largely occurs due to low energy electrons (3-50 keV) which do not penetrate the external surface of the spacecraft 27. The ultimate sources of these charges are trapped charged particles of the van Allen radiation belts, galactic cosmic rays, and the solar wind. This deposited charge accumulates and can lead to significant potential differences 
between parts of a spacecraft, sometimes leading to breakdown and discharge called Paschen discharge. Deep dielectric charging occurs when higher energy charged particles penetrate the surface of a dielectric material, which can also lead to large potential differences if the dielectric leakage is lower than the external charging rate. In a fluid mechanical context, this accumulated charge could be problematic during any multiphase venting process, as might be expected to occur during autonomous satellite servicing refueling operations, or during boil-off venting of cryogens stored over long periods in a space environment (as in many crewed Mars mission scenarios, or in cryogenic propellant depot operations). Such venting, if occurring in a region of strong field, will also tend to produce a stream of charged drops, which will be decelerated by the field, causing their eventual return, impacting and potentially contaminating the spacecraft surfaces.

As previously noted, robust phase separation is critical to high reliability multiphase systems used in thermal control and life support. Active solidstate phase separation for life support multiphase flows, especially high voidfraction droplet disperse flows is a possible application of this work. Such flows are encountered in dish washing, laundry, waste solids drying, food processing, the Sabatier $\mathrm{CO}_{2}$ reaction, and possibly in vapor-compression cycle condensers. Phase separation for other droplet disperse flows include electrostatic droplet separators for high-efficiency Rankine-cycle turbines, which require a droplet-free vapor phase entering the turbine, but where conventional superheat approaches come with severe mass penalties [28]. More speculatively, results of this work could be applied in high temperature liquid droplet radiators with electrostatic collection [29]. Removal of satellite 
droplets produced during pipetting in wet-lab research outside of a glovebox environment aboard ISS has also been recently suggested as a application of the work [private communication, NASA JSC, E. Unger, 2017]. Drops can become spontaneously charged by contact with standard micropipette tips [30], and this free charge can possibly be leveraged for the purposes of phase separation in low-gravity.

\subsection{Overview of this Thesis}

In this thesis we develop and partly validate a simple model to aid in design of future electrostatic disperse drop phase separators. To this end the work encompasses (1) a mathematical model of the electro-drop bounce, its scale analysis, asymptotic estimates for drop apoapses and times-of-flight, and (2) the results of controlled experiments, where the effect of the key independent variables of drop volume $V_{d}$ and dielectric surface charge density $\sigma$ on drop trajectories is tested. 


\section{Chapter 2}

\section{Theory}

\subsection{Equation of Motion}

We develop here a simple 1-dimensional model of the dynamics of drops dominated by electrostatic forces. We treat a drop as a particle with radius $R_{d}$, which translates vertically along the central axis of a charged dielectric square sheet substrate with initial velocity $U_{0}$. The equation of motion for this system is given by,

$$
m y^{\prime \prime}=-\mathbf{F}_{D}-\mathbf{F}_{E}
$$

subject to

$$
y(0)=R_{d}, \quad \text { and } \quad y^{\prime}(0)=U_{0},
$$

where $m$ is the drop mass, $y^{\prime \prime}=\frac{d^{2} y}{d t^{2}}$ is the drop acceleration, $\mathbf{F}_{D}$ is the drag force which always opposes motion, and $\mathbf{F}_{E}$ is the electrostatic force. The assumed initial conditions are such that, when $\mathbb{B}$ o is suddenly reduced at the start of the drop tower free-fall period, the drop jumps with instantaneous initial velocity $U_{0}$ from its $1-g_{0}$ resting position with fixed radius $R_{d}$ at $t=0$. The dynamical system is sketched schematically Figure 2.1. We now define models for each of the forces in this equation. 


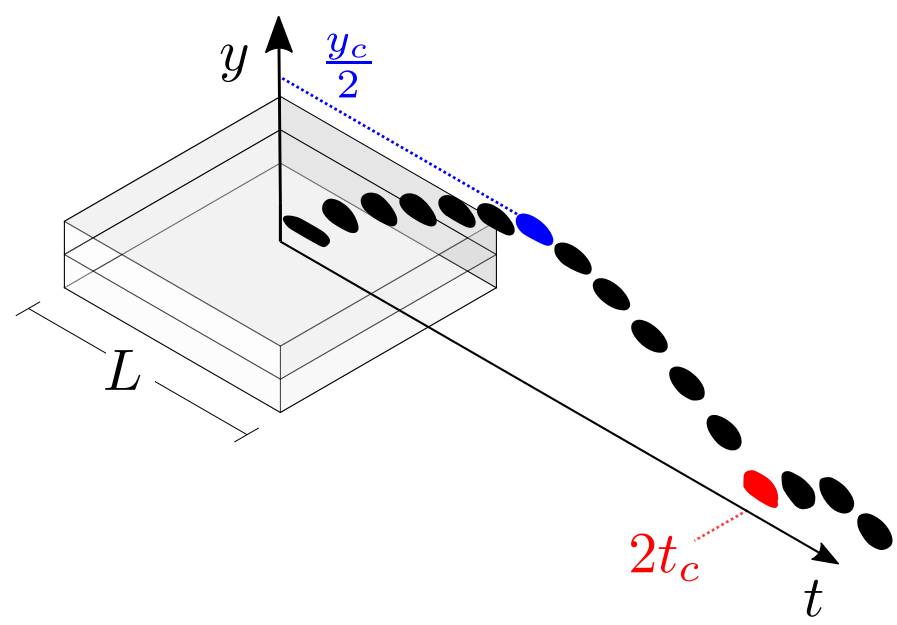

Figure 2.1: Schematic representation of drop jump with return and rebound from an electrically charged superhydrophobic substrate. The characteristic time and length scales $t_{c}$ and $y_{c}$ describe the time of flight and apoapse associated with the drop trajectory.

For the intermediate range of Reynolds numbers $1 \leq \mathbb{R e} \equiv \frac{2 U R_{d}}{\nu} \leq 2000$ observed in our experiments, the force of drag acting on the drop may be quadratic such that,

$$
\mathbf{F}_{D}=\frac{1}{2} C_{D} \rho A y^{\prime 2}
$$

where $C_{D}$ is the drag coefficient, $\rho$ is the density of the air host fluid, and $A$ is the frontal projected area of the drop. For this range of Reynolds numbers the drag coefficient may be approximated by the well known Abraham correlation 31],

$$
C_{D}=\frac{24}{9.06^{2}}\left(1+\frac{9.06}{\sqrt{\mathbb{R e}}}\right)^{2}
$$

Modeling the electrostatic force is more involved, but we begin with the standard electrohydrodynamic (EHD) approximation [32]. Under a DC electric field, we assume that the real part of the dielectric permittivity $\epsilon$, $\operatorname{Re}(\epsilon) \approx$ constant. We also assume that electric currents are small enough 
that the effects of magnetic fields can be neglected. The validity of this assumption rests on the characteristic time scale $\tau_{e}=\epsilon / \sigma_{e} \ll 1$, where $\tau_{e}$ is the ratio of absolute dielectric permittivity $\epsilon=\kappa \epsilon_{0}$, to conductivity $\sigma_{e}$, of the medium, $\kappa$ is the relative dielectric permittivity, and $\epsilon_{0}$ is the vacuum permittivity. This characteristic time $\tau_{e}$ is also known as the relaxation time, and is a measure of how quickly the polarization of a dielectric responds to a change in electric field. Given the conductivity and permittivity in the limiting case of extremely-pure water $\left(\epsilon \sim 80, \sigma_{e}=18.2 \times 10^{6} \Omega \mathrm{cm}\right)[22]$, we estimate $\tau_{e} \approx 4 \times 10^{-6} \mathrm{~s}$. The relaxation time for the common distilled water that is actually used in the drop experiments is undoubtedly shorter due to the presence of solvated ions. Neglecting the effects of an electric double layer on hydration of ions in the water or the ambient atmosphere due to the relatively massive size of the drops studied, the assumption of small relaxation time further implies that the free charge present in the drops will remain approximately constant during the typical time interval of a low-gravity experiment.

Supposing that electrical forces acting on free charges and dipoles in a fluid are transferred directly to the fluid itself, the overall electrical body force will be the divergence of the Maxwell stress tensor $\tau_{m}$,

$$
\mathbf{F}_{E}=\nabla \cdot \tau_{m}=\nabla \cdot\left(\epsilon \mathbf{E E}-\frac{1}{2} \epsilon \mathbf{E} \cdot \mathbf{E} \delta\right)
$$

where $\mathbf{F}_{E}$ is the electric body force per unit volume, and $\delta$ is the Dirac delta function. The product of the electric field vectors is the dyadic product.

The Korteweg-Helmholtz volumetric force density formulation of the Maxwell 
stress tensor is usually expressed as 33

$$
\mathbf{F}_{E}=\rho_{f} \mathbf{E}+\frac{1}{2}|E|^{2} \nabla \epsilon-\nabla\left(\frac{1}{2} \rho\left(\frac{\partial \epsilon}{\partial \rho}\right)_{T}|E|^{2}\right)
$$

The first term on the right hand side of this expression is the well known Coulombic force or electrophoretic force, which arises from the presence of free charge in an external electric field. We expect this term to dominate the electric force in a DC field. The second term is the force arising from polarization stresses due to a nonuniform field acting across a gradient in permittivity. This force is widely termed the dielectrophoretic (DEP) force. The third term describes forces due to electrostriction. It has been noted by Melcher and Hurwitz [10] that the electrostriction term is the gradient of a scalar and can thus be canonically lumped together with the hydrostatic pressure for incompressible fluids. We neglect it in our analysis.

It is common to approximate the polarization stress by idealizing the drop as a simple dipole using the effective dipole moment method first described by Pohl in 1958 [34]. This approach has since been related back to the force density by the volumetric integration of the force density with the substitution of a Taylor series expansion of $\mathbf{E}$ in the limit of a small radial gradient of the field within the dielectric sphere [35]. The DEP force arises due to the induced or permanent dipole moment of polarizable media which has a tendency to align the dipole with the electric field. If there is a gradient in the field then for a finite separation of charge, one end of the dipole will feel a stronger electric field than the other resulting in a net force. Whether the force is positive or negative in the direction of the electric field gradient de- 
pends on the difference of dielectric permittivites between the fluids, rather than on the polarity of $\mathbf{E}$. In principle an external electric field will tend to induce a dipole in a dielectric material, but if the field is spatially uniform there is no gradient in the field and the forces felt by the dipoles are symmetric with no net force. The dipole moment $\mu$ of a spherical linear-dielectric particle immersed in a dielectric medium is given by

$$
\mu=V_{d} \mathbf{P}=\frac{4}{3} \pi R_{d}^{3} \mathbf{P}
$$

where $\mathbf{P}=\left(\kappa_{a}-1\right) \epsilon_{0} \mathbf{E}=\chi_{e} \epsilon_{0} \mathbf{E}$ is the polarization moment, $R_{d}$ is the particle radius, $\kappa_{a}=\frac{\epsilon_{a}}{\epsilon_{0}}$ is the relative dielectric constant of the air host fluid, and $\chi_{e}=\kappa_{a}-1$ is the electric susceptibility of the host fluid. The force felt by the dipole is

$$
\begin{aligned}
\mathbf{F}_{D E P} & =\left(\mathbf{P}_{e} \cdot \nabla\right) \mathbf{E} \\
& =2 \pi R_{d}^{3} \kappa_{w} \epsilon_{0} K \nabla E^{2},
\end{aligned}
$$

where $\mathbf{P}_{e}=\left(\kappa_{w}-\kappa_{a}\right) \epsilon_{0} \mathbf{E}$ is the excess polarization and $\kappa_{w}$ is the relative dielectric constant of the water particle. Here it is convenient to use the simplifying shorthand $K=\frac{\kappa_{w}-\kappa_{a}}{\kappa_{w}+2 \kappa_{a}}$, known as the Clausius-Mossotti factor. In cases where $K<0$, or $K>0$ the particle will be either repelled or attracted to regions of strong field. In our experiment, choosing the relative dielectric constants $\kappa_{a} \approx 1$ and $\kappa_{w} \approx 80$, we estimate $K \approx 0.96$. It is important to note that the equivalent dipole approximation critically requires an assumption of small physical scale of the particle relative to the length 
scale of nonuniformity of the field, which in this case we take to be the length of the charged superhydrophobic surface, $L=25 \mathrm{~mm} \gg R_{d} \approx 2 \mathrm{~mm}$.

When the drop is close to the dielectric surface, the free charge on the drop will tend to induce polarization of the dielectric which perturbs the electric field. The polarization bound charge in the dielectric will be of the opposite sign of the free drop charge and thus there will be a force of attraction. This so-called image force is a correction to the Coulomb force due to the external electric field only, and can be found by a Green's Function solution of Laplace's equation for the electric field called the 'method of images' 36. This resulting image force $\mathbf{F}_{I}$ is given by

$$
\mathbf{F}_{I}=\frac{k q^{2}}{16 \pi \epsilon_{0}} y^{-2} \hat{\mathbf{j}}
$$

where the factor $k$ is a function of the dielectric surface susceptibility $k=$ $\frac{\chi_{e}}{\chi_{e}+2}$, and $\hat{\mathbf{j}}$ is a unit vector normal to the dielectric surface.

By substituting Equations 2.5, and 2.6 into 2.3 we have

$$
\begin{aligned}
\mathbf{F}_{E} & =q \mathbf{E}+\mathbf{F}_{D E P}+\mathbf{F}_{I} \\
& =q \mathbf{E}+\frac{k q^{2}}{16 \pi \epsilon_{0}} y^{-2} \hat{\mathbf{j}}+2 \pi R_{d}^{3} \kappa_{w} \epsilon_{0} K \nabla E^{2},
\end{aligned}
$$

and the 1-D governing equation becomes

$$
m y^{\prime \prime}=-\frac{1}{2} C_{D} \rho A y^{\prime 2}-q E-\frac{k q^{2}}{16 \pi \epsilon_{0}} y^{-2}-2 \pi R_{d}^{3} \kappa_{w} \epsilon_{0} K \nabla E^{2}
$$

subject to

$$
y(0)=R, \quad \text { and } \quad y^{\prime}(0)=U_{0}
$$


By comparing DEP and Coulombic terms in Equation 2.7, we note that a condition to neglect the DEP force is

$$
\frac{\kappa_{w} \epsilon_{0} K R_{d}^{2} E_{0}}{q} \ll 1
$$

As this condition likely prevails in our experiments we henceforth neglect the DEP force. There is some physical intuition to support this conclusion as well. The dielectric displacement $\mathbf{D}=\kappa \epsilon_{0} \mathbf{E}$ of a water drop in air is high due to its large relative dielectric constant. This implies that the field strength within the drop is about 80 times smaller than in the surrounding medium, which is essentially the same as a vacuum from a dielectric standpoint. Thus it is not particularly inaccurate to model the dielectric volume of a drop as an equipotential conductive shell with zero field in its interior. As an aside, in treating the drop as an ideal conductor we note that in our specific case the electrostatic force is not a body force per se as the electric field is acting on charges on the surface of the conductor.

\section{$2.2 \quad$ The Electric Field}

If we consider the charged dielectric surface of our experiments to be a square sheet of charge lying in the $x z$-plane with width $L$, the symmetry of the problem happily lets us obtain the $y$-component of the electric field $\mathbf{E}$ by direct integration. In particular, it is easy to construct the electric field due to a finite plane of charge by superposition of the electric fields of a series of line charges. By symmetry, the electric field points along the $y$-axis; for a point along the $y$-axis the position vector is $\mathbf{r}=\left(x^{2}+y^{2}\right)^{1 / 2} \hat{\mathbf{r}}$ to the 
center of a line charge in the $x z$-plane. The $y$-component of $d \mathbf{E}$ is found by $d E_{r}=d E_{y} \cos \theta=d E_{y} y / r$, where $\theta$ is the angle made between the $y$-axis and the position vector $\mathbf{r}$. If the charge in a line element $d x$ is $\sigma L d x$, where $\sigma$ is the average surface charge density, the electric field of a line charge is given by 36

$$
d E_{r}=\frac{\sigma L d x}{4 \pi \epsilon_{0} r \sqrt{r^{2}+L^{2} / 4}} .
$$

The $y$-component of the electric field $E_{y}$, hence $E$, is then

$$
E=\frac{\sigma L y}{4 \pi \epsilon_{0}} \int_{-L / 2}^{L / 2} \frac{1}{\left(y^{2}+x^{2}\right) \sqrt{y^{2}+x^{2}+L^{2} / 4}} d x
$$

With some substitutions the above can be integrated to obtain an expression for the electric field in terms of $y$,

$$
E=\frac{\sigma}{\pi \epsilon_{0}} \tan ^{-1}\left(\frac{L^{2}}{y \sqrt{2 L^{2}+4 y^{2}}}\right)
$$

We note that this model of the electric field is valid when $R_{d} \ll L$, which will be true for cases of small drops 'far' from the dielectric surface. For accurate models of the field in the case $R_{d} / L \sim \mathcal{O}(1)$ we likely need to numerically solve Laplace's equation for the electric potential $\varphi$,

$$
-\nabla^{2} \varphi=0
$$

By taking Taylor series expansions in large and small limits we can intuit a bit about the behavior of this field. In the limit $y / L \ll 1$ Equation 2.9 
reduces to

$$
E \approx \frac{\sigma}{4 \pi \epsilon_{0}}=E_{0}
$$

where $E_{0}$ is the characteristic electric field given by $E_{0}=\frac{\sigma}{4 \pi \epsilon_{0}}$. This field is constant and equivalent to the electric field due to an infinite plane of charge. In the limit of $y / L \gg 1$, Equation 2.9 reduces to the familiar electric field due to a point charge

$$
E \approx L^{2} E_{0} y^{-2}
$$

Both regimes given by Equations 2.10 and 2.11 can be clearly seen in Figure 2.2 .

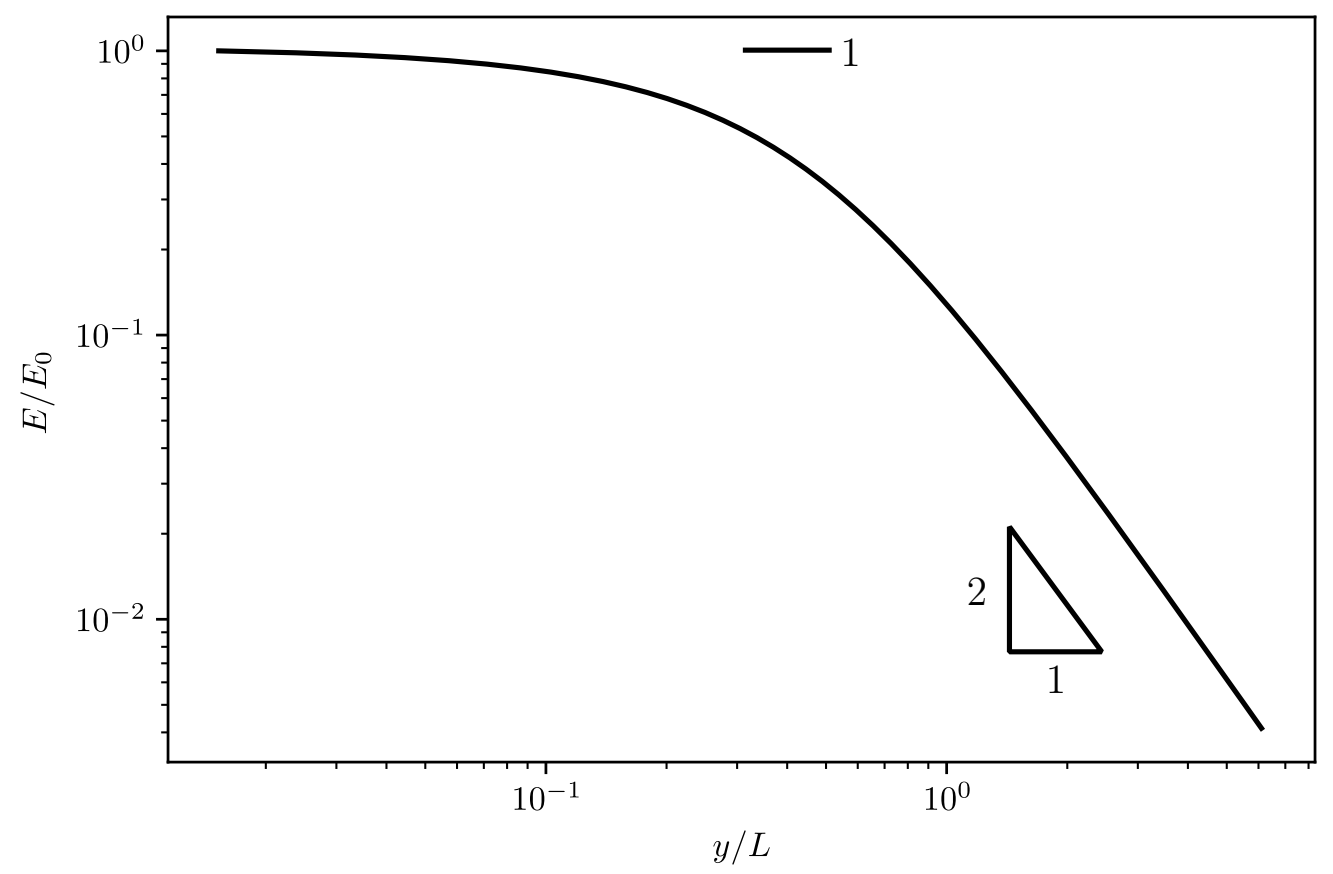

Figure 2.2: A log-log plot of the magnitude of the dimensionless electric field E. 


\section{$2.3 \quad$ Scaling}

For small DEP forces the equation of motion Equation 2.7 is non-linear, nonhomogeneous and must be solved numerically. Nevertheless we are curious if we can obtain approximate solutions to the equation analytically using perturbation methods. This is aided by non-dimensionalizing the governing equations, forming dimensionless groups, and determining whether any are particularly small or large, and whether any fortuitous opportunities for simplification appear. Introducing the scaled variables

$$
t^{*}=\frac{t}{t_{c}}, \quad y^{*}=\frac{y}{y_{c}}
$$

where $y_{c}$ and $t_{c}$ are characteristic length and time scales respectively, and using the coordinate transformation $y(0)-R=0$, the governing equation becomes

$$
y^{* \prime \prime}=-\frac{1}{2} \frac{C_{D} \rho A y_{c}}{m} y^{* \prime 2}-\frac{q E_{0} t_{c}^{2}}{m y_{c}} E^{*}\left(y^{*}\right)-\frac{k q^{2} t_{c}^{2}}{16 \pi \epsilon_{0} R^{2} m y_{c}}\left(\frac{y_{c}}{R} y^{*}+1\right)^{-2},
$$

subject to

$$
y^{*}(0)=0, \quad \text { and } \quad y^{* \prime}(0)=\frac{U_{0} t_{c}}{y_{c}} .
$$

From Equation 2.13 we note the existence of several dimensionless groups

$$
\boldsymbol{\Pi}_{1}=\frac{C_{D} \rho A y_{c}}{2 m}, \quad \Pi_{2}=\frac{q E_{0} t_{c}^{2}}{m y_{c}}, \quad \Pi_{3}=\frac{k q^{2} t_{c}^{2}}{16 \pi \epsilon_{0} R^{2} m y_{c}}, \quad \Pi_{4}=\frac{y_{c}}{R}, \quad \Pi_{5}=\frac{U_{0} t_{c}}{y_{c}},
$$


such that Equation 2.13 becomes

$$
y^{* \prime \prime}=-\Pi_{1} y^{* 2}-\Pi_{2} E^{*}\left(y^{*}\right)-\Pi_{3}\left(\Pi_{4} y^{*}+1\right)^{-2},
$$

subject to

$$
y^{*}(0)=0, \quad \text { and } \quad y^{* \prime}(0)=\Pi_{5}
$$

\subsubsection{Inertial Electro-Image Limit}

In the limit of small $y$ and $t$ we expect inertia to scale with Coulombic and image forces. In this limit we can approximate the electric field as the constant $E_{0}$. One possible characteristic length scale is $y_{c} \sim R_{d}$, however this scale is overly restrictive with respect to time. With $y_{c} \sim U_{0} t_{c}$ and picking $t_{c}$ such that the Coulombic force $\Pi_{3} \sim \mathcal{O}(1)$, the intrinsic scales are found such that

$$
t_{c} \sim \frac{m U_{0}}{q E_{0}}, \quad \text { and } \quad y_{c} \sim \frac{m U_{0}^{2}}{q E_{0}}
$$

With these scales the governing equation 2.14 becomes

$$
y^{* \prime \prime}=-1-\mathbb{I g}\left(\mathbb{E} u y^{*}+1\right)^{-2},
$$

subject to

$$
y^{*}(0)=0, \quad \text { and } \quad y^{* \prime}(0)=1 \text {. }
$$

with

$$
\mathbb{I g} \equiv \frac{k q}{16 \pi \epsilon_{0} R_{d}^{2} E_{0}}=\Pi_{3}, \quad \mathbb{E} \mathrm{u} \equiv \frac{m U_{0}^{2}}{q E_{0} R_{d}}=\boldsymbol{\Pi}_{4}
$$


where the Image number $\mathbb{I g}$ is the ratio of image forces to the Coulombic force of the unperturbed field, and the electrostatic Euler number $\mathbb{E} u$ is the ratio of inertia to electrostatic force. With these dimensionless groups the intrinsic scales become

$$
t_{c}=\mathbb{E u} \frac{R_{d}}{U_{0}}, \quad \text { and } \quad y_{c}=\mathbb{E} \mathrm{u} R_{d}
$$

\subsubsection{Inertial Electro-Viscous Limit}

In the limit of large $y$ and $t$ we expect drop inertia to balance Coulombic force and drag. In this limit we approximate the electric field as $E \approx y_{c}^{2} E_{0} y^{-2}$. In this case there are several obvious possible choices of scales:

1. $y_{c} \sim U_{0} t_{c}$ and $\Pi_{3} \sim \mathcal{O}(1)$

2. $y_{c} \sim L$ and $t_{c} \sim \frac{L}{U_{0}}$ (making Equation 2.13 singular)

3. $y_{c} \sim L$ and $t_{c} \sim\left(\frac{L m}{q E_{0}}\right)^{1 / 2}$

4. $y_{c} \sim L$ and $\Pi_{3} \sim \mathcal{O}(1)$

We choose the Case 1 scaling for its combination of physical simplicity, few $\Pi$ terms, and homogeneous initial conditions. The intrinsic scales for this case are given by

$$
t_{c} \sim \frac{R_{d}^{2}}{L^{2}} \frac{4 \pi m U_{0}}{q E_{0}} \quad \text { and } \quad y_{c} \sim \frac{R_{d}^{2}}{L^{2}} \frac{4 \pi m U_{0}^{2}}{q E_{0}}
$$

With this scaling the non-dimensional governing equation is

$$
y^{* \prime \prime}=-\mathbb{D g} \phi \mathbb{E} u y^{* \prime 2}-\left(\phi \mathbb{E} u y^{*}+1\right)^{-2}
$$


subject to

$$
y^{*}(0)=0, \quad \text { and } \quad y^{* \prime}(0)=1,
$$

where we call $\mathbb{D} g$ the drag number $\mathbb{D g} \equiv \frac{C_{D} \rho_{a}}{\rho_{l}}=\Pi_{1} \phi^{-1} \mathbb{E} \mathrm{u}^{-1}$, and $\phi=4 \pi \frac{R_{d}^{2}}{L^{2}}$ is a dimensionless ratio of length scales.

\subsection{Asymptotic Estimates}

\subsubsection{Inertial Electro-Image Limit}

The alternate scalings of the equation of motion given by Equations 2.15 and 2.16 are weakly non-linear differential equations in the sense that they reduce to linear equations as the parameter $\mathbb{E} u \rightarrow 0$. If we take $\mathbb{E} u$ to be a small parameter we can find an asymptotic approximation to the solution of the non-linear equation by means of a regular perturbation. In this case we use the naive expansion

$$
y^{*}\left(t^{*}\right) \sim y_{0}^{*}\left(t^{*}\right)+\mathbb{E u} y_{1}^{*}\left(t^{*}\right)+\mathbb{E u}^{2} y_{2}^{*}\left(t^{*}\right) \ldots \mathbb{E} u^{n} y_{n}^{*}\left(t^{*}\right)
$$

By substitution of Equation 2.17 and its derivatives into 2.14, and equating terms by order we first find the $\mathcal{O}(1)$ unperturbed solution

$$
y_{0}^{*}\left(t^{*}\right)=t^{*}+\frac{t^{* 2}}{2}(-1-\mathbb{I g})
$$

Looking at this solution it is evident that if $\mathbb{I g}=0$, the solution is the classical kinematic equation for projectile motion without drag under constant gravity $g_{0}$. Continuing on with this procedure we find, after some tedious 
computations documented in the project repository for this thesis [37], the $\mathcal{O}\left(\mathbb{E u}^{5}\right)$ order accurate solution truncated to $\mathcal{O}\left(\mathbb{E u}^{2}\right)$ is

$$
\begin{gathered}
y^{*}\left(t^{*}\right)=t^{*}+\frac{t^{* 2}}{2}(-1-\mathbb{I g})+\mathbb{E} u\left(\frac{\mathbb{I g} t^{* 3}}{3}+\frac{\mathbb{I g} t^{* 4}}{12}(-1-\mathbb{I g})\right) \\
+\mathbb{E} \mathrm{u}^{2}\left(-\frac{\mathbb{I g} t^{* 4}}{4}+\frac{\mathbb{I g} t^{*} 5}{60}(9+11 \mathbb{I g})+\frac{\mathbb{I g} t^{* 6}}{360}\left(-9-20 \mathbb{I g}-11 \mathbb{I g} g^{2}\right)\right)+\mathcal{O}\left(\mathbb{E} u^{3}\right) .
\end{gathered}
$$

We plot the approximate short-time scaled solution Equation 2.18 with varying values of $\mathbb{I g}$ in Figure 2.3. These plots show a trend of decreasing time-

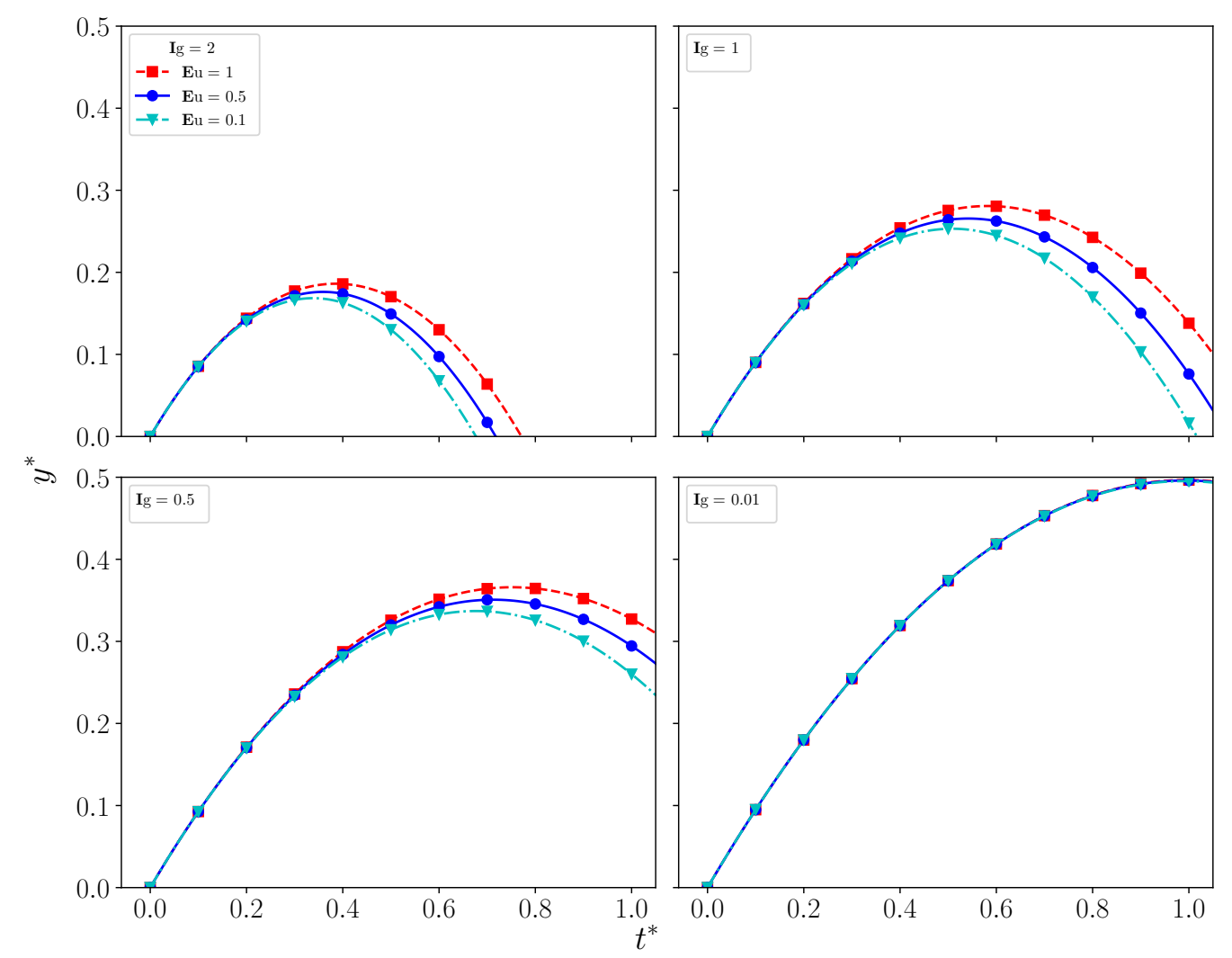

Figure 2.3: Short-time scaled drop trajectories for various values of $\mathbb{E} u, \mathbb{I g}$. The trajectory reduces to the classical $\mathcal{O}(1)$ solution for small values of $\mathbb{I g}$. It should be noted that despite what is implied by these plots $\mathbb{I g}$ is not necessarily independent of $\mathbb{E u}$, as they share $q$, and $E_{0}$ as common factors, $U_{0} \propto R_{d}^{2}$, and $m \propto R_{d}^{3}$. 
of-flight $t_{f}$, which is the time for the drop to return to the origin (a single 'bounce'), and height at apoapse with increasing values of $\mathbb{I g}$. When $\mathbb{I g}=1$, $t_{f}$ is exactly half of the characteristic time scale in this regime. In the limit of small $\mathbb{I m}$ the trajectories collapse to the $\mathcal{O}(1)$ solution regardless of the electrostatic Euler number. Trajectories with $\mathbb{E u} \leq 0.1$ are essentially coincident given the scale of the axes used here. In principle there is some coupling between $\mathbb{E u}$ and $\mathbb{I g}$; notably this relationship does not depend on the electric field $E_{0}$ but on a charge to mass ratio. The effect of contact line hysteresis on the initial jump velocity $U_{0}$ will also tend to decohere the natural covariance between these parameters.

\subsubsection{Inertial Electro-Viscous Limit}

By similar arguments we find an asymptotic estimate of the trajectory in the long-time regime. The approximate solution is

$$
\begin{gathered}
y^{*}\left(t^{*}\right)=t^{*}-\frac{t^{* 2}}{2}+\phi \mathbb{E u}\left(\frac{t^{* 3}}{3}(1+\mathbb{D g})+\frac{t^{* 4}}{12}(-1-\mathbb{D g})-\frac{\mathbb{D} g t^{* 2}}{2}\right) \\
+\phi^{2} \mathbb{E u}^{2}\left(\frac{t^{* 4}}{12}\left(-3-3 \mathbb{D} \mathrm{g}-4 \mathbb{D} \mathrm{g}^{2}\right)+\frac{t^{* 5}}{60}\left(11+10 \mathbb{D g}+8 \mathbb{D} \mathrm{g}^{2}\right)\right. \\
\left.+\frac{t^{* 6}}{360}\left(-11-10 \mathbb{D} \mathrm{g}-8 \mathbb{D} \mathrm{g}^{2}\right)+\frac{\mathbb{D} \mathrm{g}^{2} t^{* 3}}{3}\right)+\mathcal{O}\left(\phi^{3} \mathbb{E} \mathrm{u}^{3}\right) .
\end{gathered}
$$

Trajectories for this solution are shown in Figure 2.4. If we assume a constant scale for the drag coefficient $C_{d} \approx 0.5$ then $\mathbb{D g}$ is approximately a constant $\mathbb{D g} \approx 6 \times 10^{-4}$ in all of our experiments. We note that the trajectory reduces to the classical $\mathcal{O}(1)$ solution for small values of of $\phi \mathbb{E} u$. We also note that with $\mathbb{D g}=6 \times 10^{-4}$ the effect of drag is slight, appearing only as a slight correction to the higher order terms. 


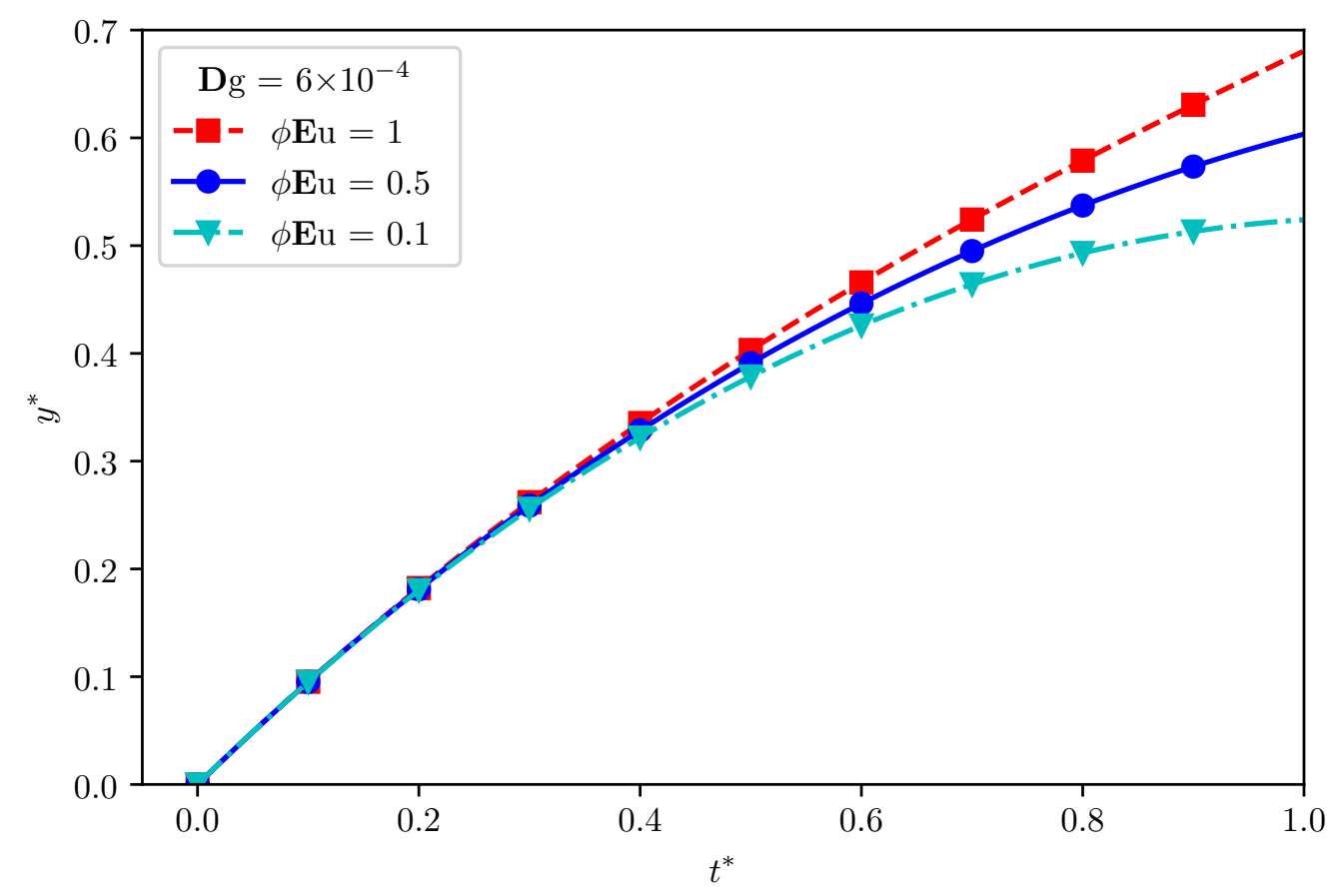

Figure 2.4: Long-time scaled drop trajectories for various values of $\phi \mathbb{E} u$.

By again applying a regular perturbation to the asymptotic solution with the expansion

$$
t^{*} \sim t_{0}^{*}+\phi \mathbb{E} u t_{1}^{*}+\phi^{2} \mathbb{E} u^{2} t_{2}^{*} \ldots \phi^{n} \mathbb{E} u^{n} t_{n}^{*}
$$

and solving for the roots at times when $y^{*}=0$, we find an asymptotic estimate for the time-of-flight. The $\mathcal{O}\left(\phi^{2} \mathbb{E} u^{2}\right)$ accurate time-of-flight estimate is given by

$$
t_{f}=2+\phi \mathbb{E} u\left(\frac{4}{3}-\frac{2 \mathbb{D g}}{3}\right)+\phi^{2} \mathbb{E} u^{2}\left(\frac{4}{5}-\frac{4 \mathbb{D g}}{3}+\frac{2 \mathbb{D g} g^{2}}{5}\right)+\mathcal{O}\left(\phi^{3} \mathbb{E} u^{3}\right)
$$

Substituting the experimental value of $\mathbb{D} g$ we find the time-of-flight estimate 
for water drops is

$$
t_{f}=2+1.333 \phi \mathbb{E} u+0.799 \phi^{2} \mathbb{E} u^{2}+\mathcal{O}\left(\phi^{3} \mathbb{E} u^{3}\right)
$$

In Figure 2.5 we see the effect of increasing values of $\phi \mathbb{E} u$ on $t_{f}$. As $\mathbb{E u}$

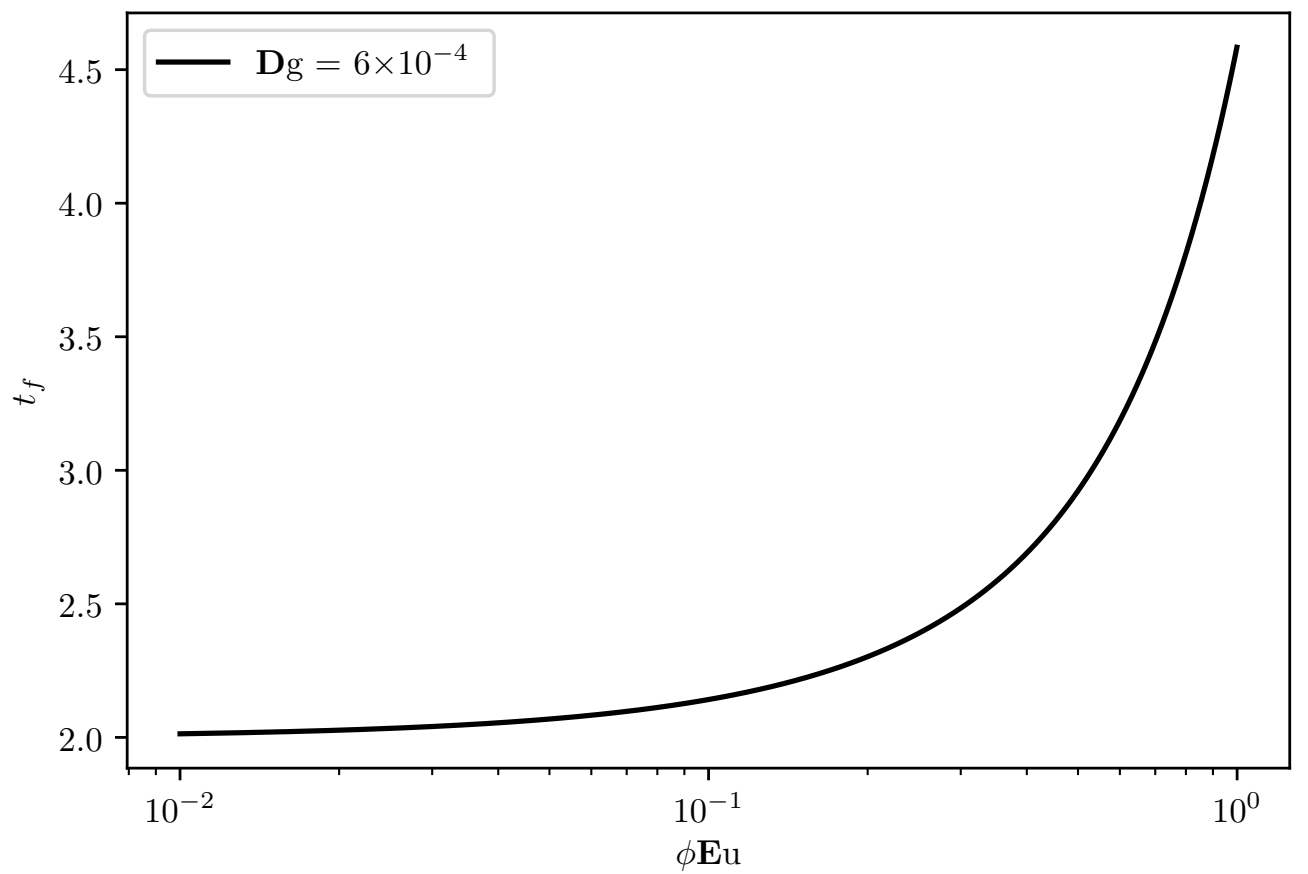

Figure 2.5: $\mathcal{O}\left(\phi^{3} \mathbb{E} u^{3}\right)$ accurate asymptotic estimate of drop time-of-flight $t_{f}$.

grows to be no longer small, the time-of-flight grows rapidly, but this behavior appears to have an asymptote at a certain critical velocity; this is an electrostatic escape velocity $U_{e}$. We can find the escape velocity by solving a modified version of the equation of motion

$$
m u^{\prime}=-\frac{q E_{0} y_{c}^{2}}{y^{2}}
$$


where $u=\frac{d y}{d t}$ is the drop velocity. This has the solution

$$
u(y)= \pm U_{0}\left(1+\frac{2 q E_{0} y_{c}^{2}}{m U_{0}^{2}}\left(\frac{1}{y}-\frac{1}{R_{d}}\right)\right)^{1 / 2}
$$

This equation has an asymptotic velocity $U_{\infty}$ at $y=\infty$, which is real provided

$$
U_{0} \geq U_{e}=y_{c} \sqrt{\frac{2 q E_{0}}{m R_{d}}}
$$

where $U_{e}$ is the escape velocity and $U_{\infty}=\sqrt{U_{0}^{2}-U_{e}^{2}}$. If $y_{c}=L$ the condition for the drops to escape the electric field is then given by

$$
\frac{1}{8 \pi} \phi \mathbb{E} \mathrm{u}>1
$$

If the drop escapes the electric field it will have a residual velocity $U_{\infty}$ and will be in a regime where inertia $\sim$ drag. The velocity will then decay by drag as

$$
u^{*}\left(t^{*}\right)=\frac{1}{t^{*}+1}
$$

with a characteristic time $t_{c} \sim \frac{2 m}{C_{D} \rho A U_{\infty}}$, which is the halving time of the velocity.

\subsection{Summary}

The objective of this work is to understand the basic physics of the lowgravity electro-drop bounce phenomenon to the point where we can make useful predictions of drop behavior for engineering purposes. To that end, in this chapter we have shown the following: 
- We develop from first principles a simple 1-dimensional model of drop trajectories subject to Coulombic forces and drag under low-gravity. Drops are idealized as point charges. We find that polarization stresses are negligible, and that water drops can be modeled as ideal conductors under the conditions of our experiments.

- By scaling arguments we find characteristic length and time scales for both a short-times regime where inertia balances Coulombic force and forces due to image charges, and a long-times regime where inertia balances Coulombic force and drag. In both regimes the key dimensionless electrostatic Euler number $\mathbb{E} u$ which is a ratio of inertia to electrostatic force, appears.

- The governing ODE is non-linear, but we find approximate analytic solutions for the drop trajectory and time of flight in both regimes by means of asymptotic expansions.

- We find a limiting initial velocity $U_{0}$ for which drop time of flight grows without bound. This electrostatic escape velocity is analogous to the gravitational escape velocity of a planet or other massive body. 


\section{Chapter 3}

\section{Methodology}

\subsection{Overview}

We are interested mainly about which parameters are important in electrostatic transport of relatively large millimetric drops in low-gravity, and what the values of the respective dimensionless numbers might be; namely, Ig and $\mathbb{E}$ u. However, it is impractical to directly measure all of the key physical quantities that appear in the dimensionless groups at once in a drop tower experiment. In particular, determination of net drop free electric charge $q$ is difficult as high-input resistance electrometers are not well-suited to the sudden $15-g_{0}$ decelerations characteristic of drop tower experiments. To find typical values of these parameters we use the following approach:

1. We vary the independent variables $V_{d}$ and $\sigma$ in a set of single-drop spontaneous drop jumps on charged dielectric superhydrophobic substrates under low-gravity conditions in a $2.1 \mathrm{~s}$ drop tower.

2. We capture high-speed video and digitize the trajectories of the drops.

3. We solve the inverse problem to find the key parameters by maximizing a statistical likelihood function between an observed trajectory and 
the trajectory predicted by a dynamical model given that certain set parameters.

A schematic representation of drop jump system statics is provided in Figure 3.1 .

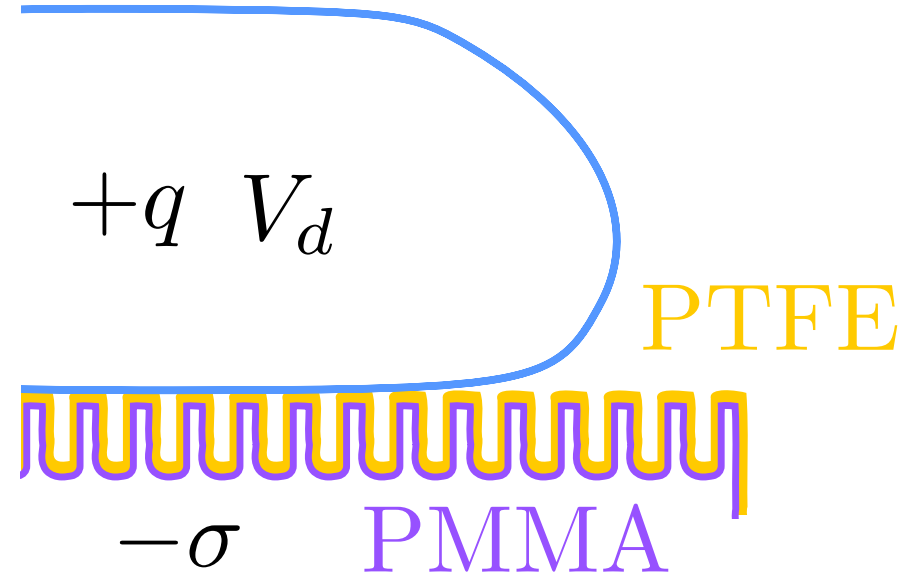

Figure 3.1: Portion of drop at the initial condition, resting on a superhydrophobic surface in the Cassie-Baxter state just prior to release of the experiment into free-fall. The variables $q, \sigma$, and $V_{d}$ are the drop net free charge, dielectric surface charge density, and drop volume, respectively.

\subsection{Experimental Methods}

The DDT uses a dual capsule design, inspired by the $2.2 \mathrm{~s}$ facility at NASA Glenn Research Center, which decouples drag acceleration felt by the external drag shield from the experiment. The experiment experiences approximately $\lesssim 1 \times 10^{-4} g_{0}$ during free-fall for 2.1 s as the rig and drag shield plummet to the bottom of drop tower 6 stories below. Single drops of distilled water in a range of volumes $\left(0.01 \leq R_{d} \leq 0.5 \mathrm{~mL}\right)$ are carefully deposited on charged superhydrophobic substrates using an grounded glass syringe with 
$\pm 1 \mu \mathrm{L}$ accuracy and then dropped in the drop tower. Red dye is added to improve thresholding accuracy in trajectory digitization. Drop trajectories are recorded using a Panasonic HC-WX970 Camera at 120 fps with 1/3000s shutter speed. In a few cases where higher frame rates are required a Nikon J1 400 fps camera with a $30 \mathrm{~mm}$ telephoto lens is used. The test cell is back illuminated with a diffused 6000K LED strip light source.

Superhydrophobic electret substrates are prepared with surface potentials $\varphi_{s}=0.7-4.0 \mathrm{kV}$. According to a review by Sessler [38], an electret is a dielectric material with quasi-permanent electric charge in the sense that the characteristic decay period of the electret is much greater than a practical experiment time scale. Electret charge may be 'true' charge in the form of surface or space charges, or polarization charges such as bound charges. If the electret is not screened by a conductor then it produces an external electric field if the polarization and real charges do not uniformly compensate each other throughout the volume of the electret. For this reason electrets are thought of as electrostatic analogs to permanent magnets. The name electret itself is a portmanteau to that effect conjured by Heaviside in 1892 [39]. Typical commercial electrets are PTFE films on the order of 10-50 $\mu$ m thick with the charge being primarily real surface charge. Electrets have a plethora of applications, but most germanely they have been used in Electro-Wetting On Dielectric (EWOD) devices for low-voltage manipulation of small drops 40]. Real charge electrets can be produced by contact electrification, injection or deposition of charge carriers by corona discharge or electron beam, ionizing radiation, or by frictional triboelectrification. Dipolar electrets by contrast are produced by a combination of polarization at elevated temperatures in 
a strong external electric field followed by an annealing process. Effective surface charge densities are limited to the material dielectric strengths due to internal dielectric breakdown phenomenon which typically occurs before external breakdown or Paschen breakdown.

We use an isothermal electret formation process which is a variation of the widely applied corona-charging technique. Typical corona-charging techniques uses strong inhomogenous DC electric fields to produce discharge in air at ambient conditions; the dielectric substrate is atop a grounded electrode and there is a screening potential electrode intermediately positioned to control the surface potential. The surface potential of the dielectric will tend to saturate at this grid potential if the material is not space-charge current limited. The corona field is usually produced by a pin-shaped electrode. In air the most common charge carriers thus produced are $\mathrm{CO}_{3}^{-}$ions. This approach is known to generally produce samples with fairly uniform surface charge densities. Some work by van Turnhout [41] using Thermally Stimulated Current (TCS) measurements showed that in $4.8 \mathrm{~mm}$ thick polymethyl methacrylate (PMMA), polarization of the dielectric is non-uniform due to real space-charge mostly $(\sim 90 \%)$ residing in a thin $(0.1-0.2 \mathrm{~mm})$ layer near the free-surface of the sample. In this work we use a Ptec IN5120 balanced $\mathrm{AC}$ corona ion source to direct a net neutral stream of ions towards the dielectric target, which we polarize by an electrode with an EMCO P20P $2 \mathrm{kV}+$ absolute reference DC-DC converter. The ion stream compensates the surface and space bound charges arising due to the polarization of the dielectric. When the DC-DC converter is switched to ground the deposited negative ions remain on the surface. 
The electret is lamina of 3 to $40.4 \mathrm{~mm}$ thick corona charged PMMA sheets. The electric field strength scales with the number of dielectric lamina as has also been shown in work on electret based vibrational energy harvesters [42] and in water desalinization [43].

The electret is established on a superhydrophobic substrate produced by laser etching PMMA and depositing a thin layer of PTFE on the roughened surface. The surface charge density can be modulated during the experiment using the high-voltage DC-DC converter, which can re-polarize the dielectric substrate by means of an embedded electrode. The resulting bound charge partially or fully neutralizes the electric field due to the surface ions deposited by corona charging of the electret. The high-voltage system is armed manually before the drop tower experiment and is automatically safed by a high-voltage power switching relay, which switches the load across a 100 $\mathrm{k} \Omega$ resistor when triggered by the resumption of $1-g_{0}$ conditions. The safing is set by an accelerometer pin-interrupt triggered microcontroller command. The rig with a mounted experiment is shown in Figure 3.2 .

Contact angles of distilled water on the electret are $\sim 150^{\circ}$. The hysteresis of the contact angle (the difference between the advancing $\theta_{a}$ and receding $\theta_{r}$ contact angles) is estimated from the roll-off angle using the method of Furmidge [44], and is found to be approximately $25^{\circ} \pm 10^{\circ}$ when the surface is uncharged. A contour plot of the Furmidge model is provided in Figure 3.3. The contact angle for water on smooth PTFE is $\approx 115^{\circ}$ and is further enhanced by an air layer maintained in the underlying roughness length scales of the surface. We use a laser-etched pillared geometry with pillar heights $\sim 775 \mu \mathrm{m}$, widths $\sim 70 \mu \mathrm{m}$, and pitch $\sim 100 \mu \mathrm{m}$. An SEM image of the pillar 


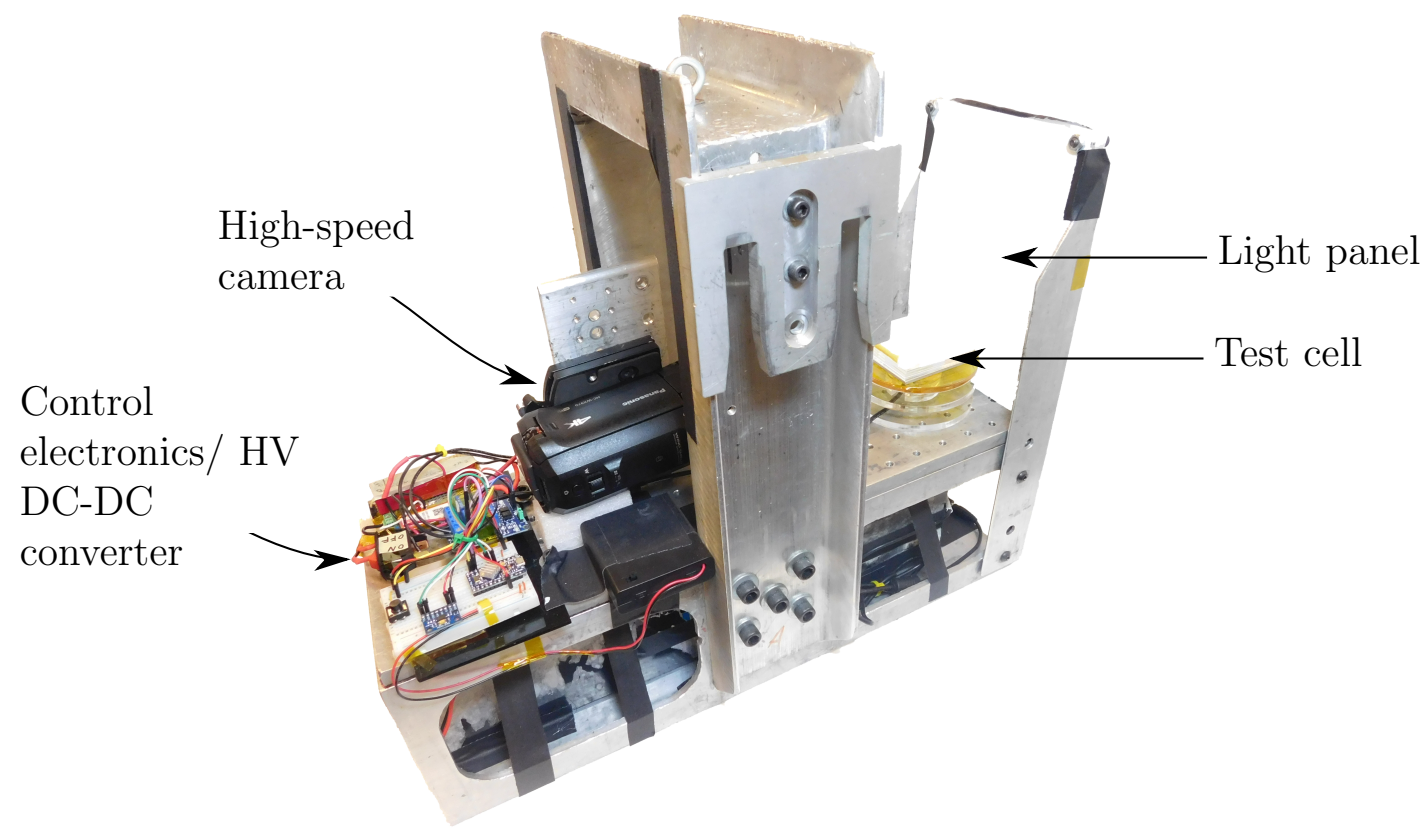

(a)

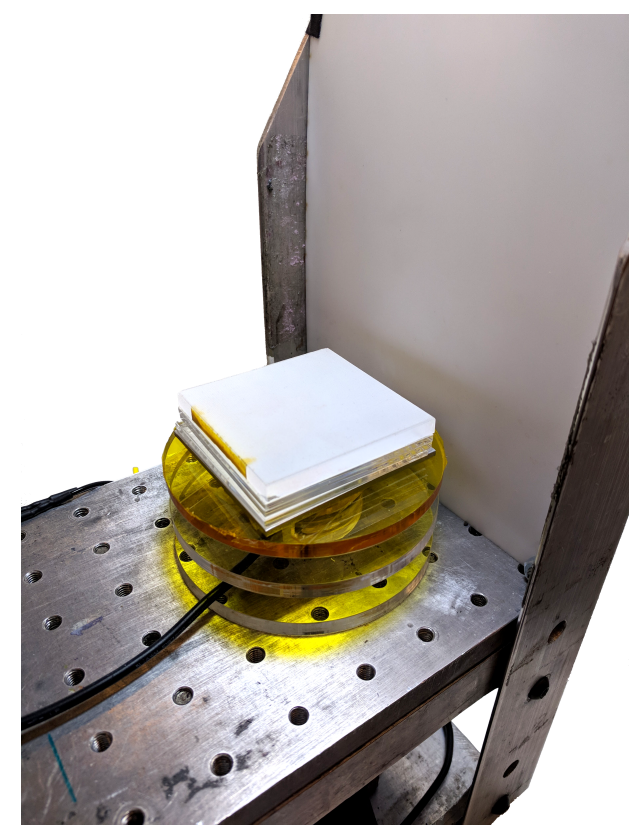

(b)

Figure 3.2: (a) The electro-drop bounce experiment hardware mounted on a drop tower rig. (b) Close up of the experimental test cell. 


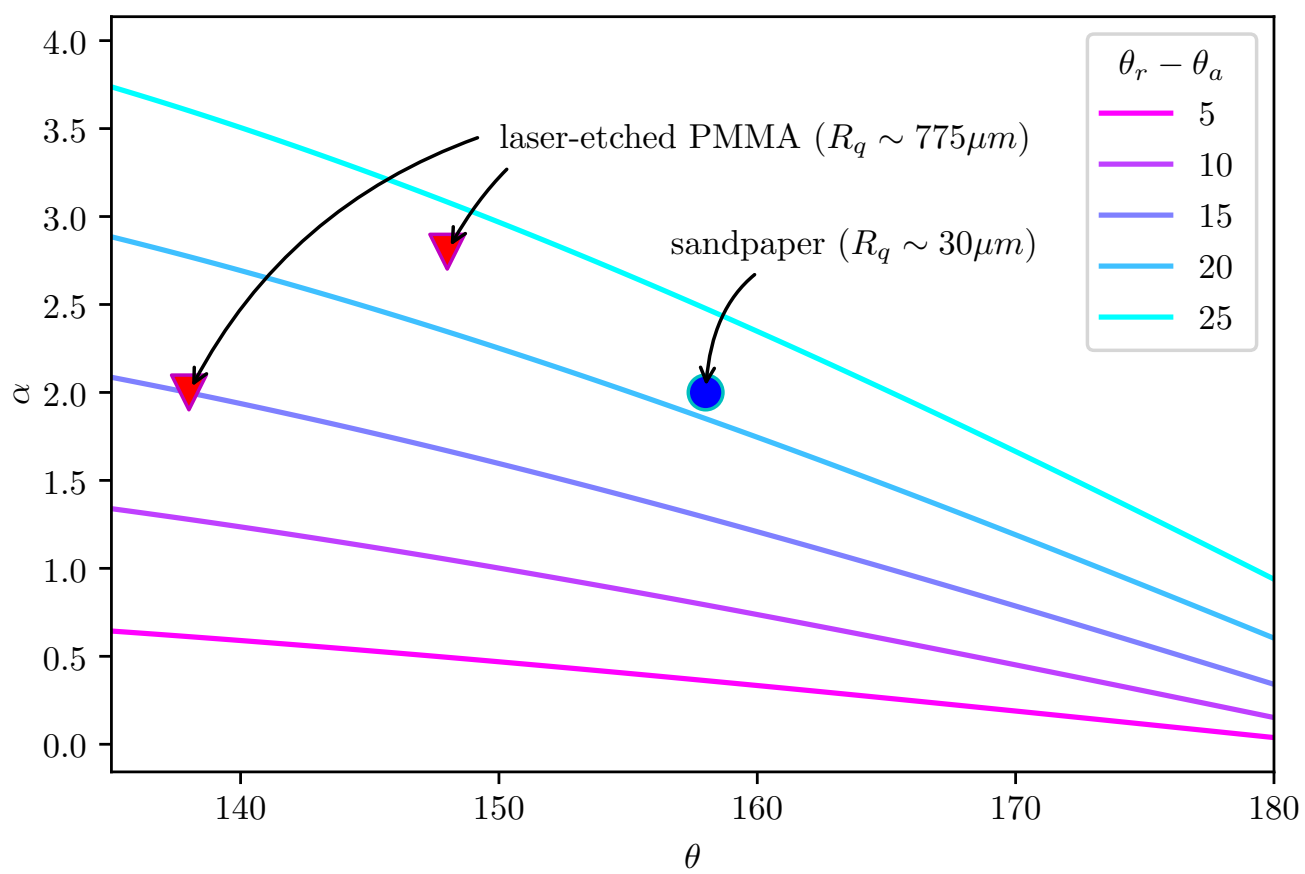

Figure 3.3: The Furmidge model of the hysteresis of the contact angle plotted as a function of static contact angle $\theta$ and roll-off angle $\alpha$ for $1 \mathrm{~mL}$ drops. The measured static contact angles and roll-off angles of several superhydrophobic surfaces including the laser-etched PMMA used in this work are also shown annotated with the characteristic roughness lengthscale $R_{q}$ of the surface.

geometry is shown in Figure 3.4 .

Surface potentials $\varphi_{s}$ are measured on the superhydrophobic surface using a Simco-Ion FMX-004 electrostatic fieldmeter and the method for determination of surface charge density for low conductivity polymers described in Davies [45]. This measurement is made with the electret substrate bonded to a conductive ground plane by conductive tape far from the presence of other conductors. An ideal approach to determining surface charge on a dielectric surface is to screen perturbing effects of external electric fields. This is partly accomplished by grounding the fieldmeter and by placing the dielectric sam- 


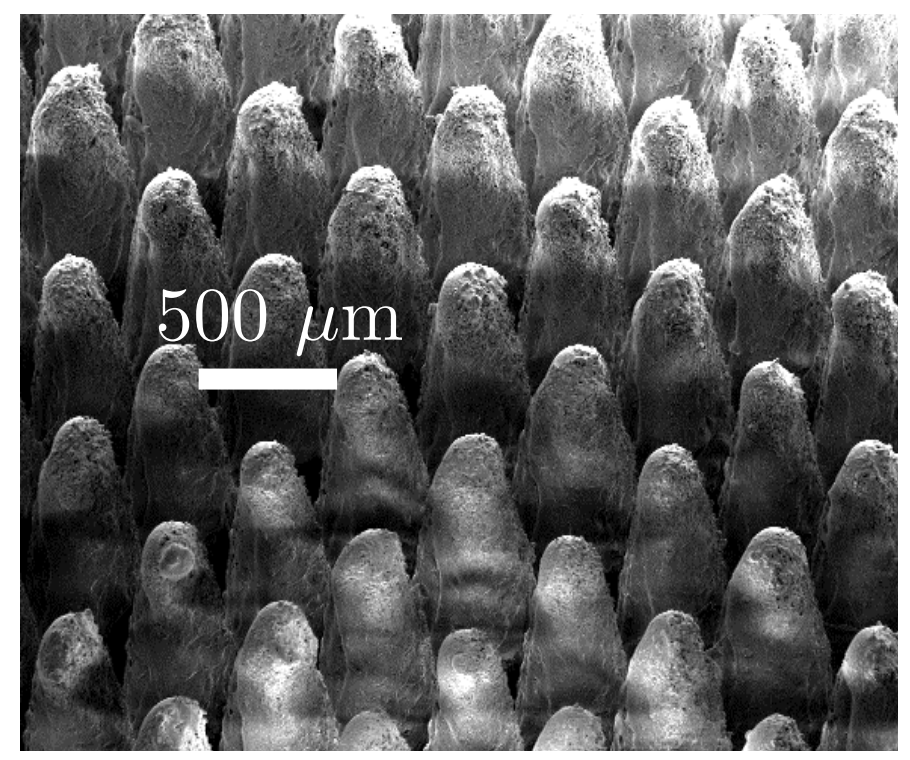

Figure 3.4: SEM image of the superhydrophobic surface.

ple on a grounded conductive plate backing. In this case the surface charge density is determined from $\sigma=\varphi_{s} \kappa \epsilon_{0} / l$, where $l$ is the thickness of the dielectric surface. The measured surface voltage is a function of position away from the charged dielectric. In most cases this function is relatively constant at a distance of about 1-2 $\mathrm{cm}$ away from the surface though we note that there is some measurement error in surface voltage due to small mispositioning of the electrostatic fieldmeter, say by $\pm 1 \mathrm{~mm}$. The relative dielectric constant of the PMMA sheet is measured by using a $65 \times 65 \mathrm{~mm}$ polished aluminum parallel plate capacitor with $C=\kappa \epsilon_{0} A / l$, where $C$ is the capacitance, and $A$ is the sheet area. Measuring the capacitance with 3 sample thicknesses using a GenRad 1657 RLC Digibridge, we find the relative permittivity to be $\kappa=3.5 \pm 0.4$.

A further consideration is the possibility of the change in total charge during a typical experimental timescale. If we consider the drop rig to be a 
ground which is reasonable given that the rig is isolated from true ground, but is at some reference voltage with respect to the surface charges on the dielectric with an abundance of free charge carriers, then there will be both bulk and surface Ohmic decay of the charge on the dielectric. The evolution of the charge can in some cases be approximated by $\sigma=\sigma_{0} e^{\frac{-t}{\epsilon \rho}}$, where $\sigma_{0}$ is the initial surface charge density and $\rho$ is the bulk resistivity. For an example case of a surface with relative permittivity $\kappa=3.5$ and bulk resistivity $\rho=1.6 \times\left(10^{16}\right) \Omega \mathrm{cm}$ such as with the case of a $2.8 \mathrm{~mm}$ PMMA sheet, then the time constant $\tau=\kappa \epsilon_{0} \rho$ is approximately $5000 \mathrm{~s}$, which is significantly longer than the typical time period for a drop tower experiment. External charge decay mechanisms include compensation by environmental ionic species. The relative magnitudes of these charge transport mechanisms, and therefore the stability of the electret, vary drastically depending on the initial surface potential, material properties, environment, and charging method. In the case of unshielded electrets compensation by atmospheric ions is significant 46. Because environmental convection will tend to maintain a gradient of these ions, sealing an electret in a container from the atmosphere will effectively halt this decay mechanism. Atmospheric humidity and water drop condensation can also significantly increase charge decay, presumably by reducing the surface resistance 47 . Examples of this decay can be seen in Figure 3.5 for differing numbers of dielectric lamina used in this experiment. In looking at the trends in charge decay for our electrets we notice firstly that the decay does not appear to be exponential, as in the model described above. Secondly, we plainly see a cross-over effect in the decay of the surface potential in our electret samples, whereby the samples charged to higher initial surface 
potentials decay faster and reached the lower overall final potentials. This is a well known effect in polyethylenes charged by corona [48].

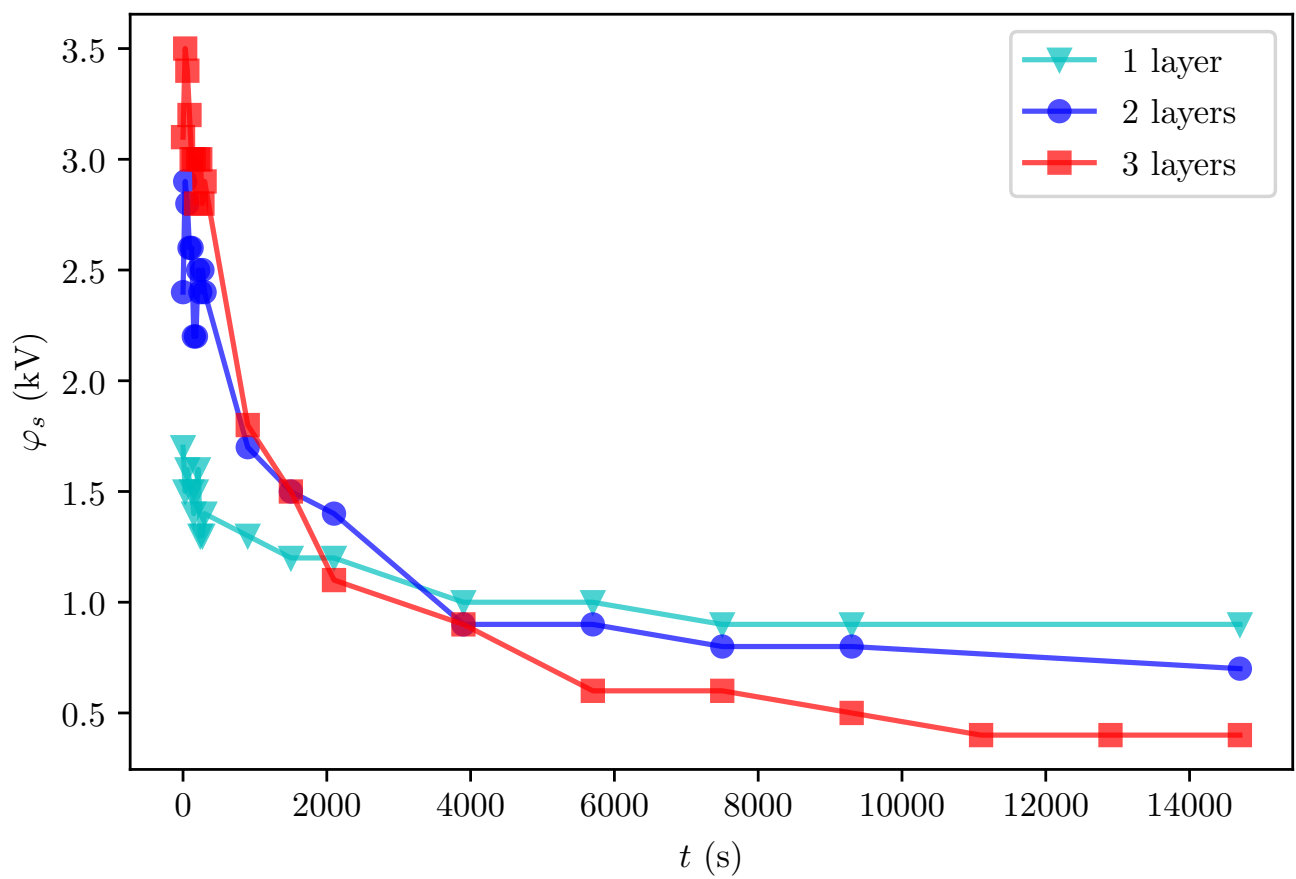

Figure 3.5: Charge decay in the dielectric laminates for several numbers of layers. 


\subsection{Data Reduction}

Digitization of drop trajectories requires several steps of post-processing. The video records are first decomposed into sequences of still images. Trajectories are captured using the particle tracking module in Fiji [49], a derivative of the popular ImageJ [50] package for scientific image analysis. The series is stabilized to remove the effect of drop transients from the kinematic data [51]. The series of still images is cropped and the low-entropy pixels are removed using a built-in rolling ball algorithm. Each still is then split into its constituent RGB maps. In this case the green channel images contain the most information. These are globally thresholded using the Triangle algorithm to recover a map of the pixels corresponding the approximate position of the drop in the original still image. Ellipses are fitted to the pixel map stepping through the time series to determine the positions of the center of mass and the semi-major and semi-minor axes of the drops during the drop tower test. Finally, a perspective correction is applied to the center-of-mass positions.

The code, raw data, and make files for this thesis are archived on the open-source project portal Github [37.

\subsection{Parameter Estimation}

Using various scaling arguments we have gleaned from our simple model a series of dimensionless numbers characteristic of drop bounce apoapses and times of flight. These dimensionless numbers depend on physical properties not all of which are easily measured by experiment. Drop free charge $q$ in 
particular could in principle be directly measured by collecting the charged drops in a Faraday cup under low-g and measuring the change in capacitance of the cup using a high input-resistance electrometer. But this method is burdensome to apply in practice in a drop tower. Other variables we can directly measure with varying levels of accuracy.

To measure the charge $q$ we must use parameter estimation techniques. Our work flow to identify $q$ in an individual drop tower experiment is as follows:

1. Experimentally vary $V_{d}$ and $\sigma$, capturing drop trajectories using a highspeed camera.

2. Digitize drop trajectories via automatic tracking of ellipse-fitted centers of mass on the thresholded video.

3. Slice drop trajectories by their bounce minima and apply a smoothing filter.

4. Extract the drop charge as well as other experimental parameters by maximizing the log-likelihood of the data given the dynamical model by varying the parameter vector using a direct search optimization.

Mathematically we state that we find the parameters $\mathbf{x}$ that solve the inverse problem $G(\mathbf{x})=\mathbf{d}$ using the Nelder-Mead direct search method to maximize the log-likelihood of the data. This is equivalent to minimizing the $\chi^{2}$ goodness-of-fit between an experimentally observed drop trajectory and the trajectory predicted by Equation 2.7 given a vector of parameters 
$\mathbf{x}=\left\langle q, V_{d}, \sigma\right\rangle$. This problem is stated as

$$
\begin{aligned}
& \min \chi^{2}=\min \sum_{i=1}^{n} \frac{\left(y_{d}(t)_{i}-y_{G}(t, \mathbf{x})_{i}\right)^{2}}{\sigma_{d i}}
\end{aligned}
$$

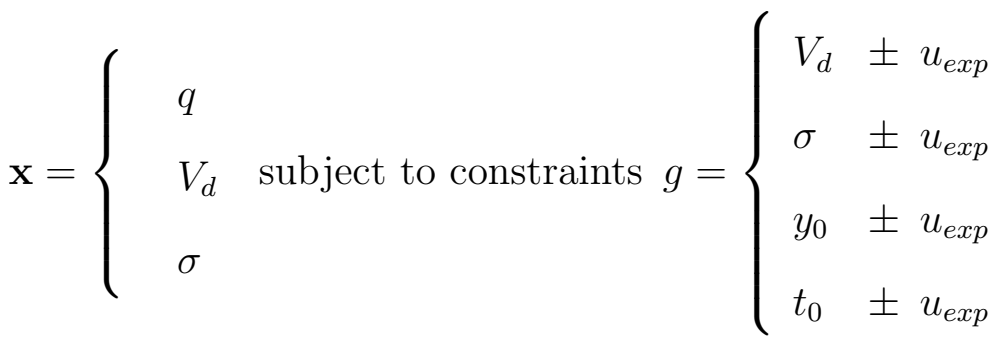

where $y_{G}(t, \mathbf{x})$ is the $y$-coordinate position at time $t$ of the numerical solution of the equation of motion

$$
m y^{\prime \prime}=\frac{1}{2} \rho C_{D} A_{d} y^{\prime 2}+q E(y)+K q^{2} y^{-2},
$$

where $y_{d}(t)$ is the corresponding experimentally observed drop $y$-coordinate position at time $t$ and $\sigma_{d}$ is the standard error of the observed position. The vector $\mathbf{x}$ that minimizes $\chi^{2}$ is the so called Maximum Likelihood Estimate (MLE) of the experimental parameters. This approach is given further explanation below.

\subsubsection{Inverse Problems}

Suppose we have a model $G(\mathbf{x})$ with a vector of parameters $\mathbf{x}$ and set of ideal observations $\mathbf{d}$. We then expect there to exist a relationship

$$
G(\mathbf{x})=\mathbf{d}
$$


Suppose the model $G(\mathbf{x})$ is the ODE

$$
y^{\prime}(t)=f(t, \mathbf{y}, \mathbf{x})
$$

and a collection of $n$ measurements of experimental data

$$
\mathbf{d}=\left(t_{1}, \mathbf{y}_{\mathbf{1}}\right),\left(t_{2}, \mathbf{y}_{\mathbf{2}}\right), \ldots,\left(t_{k}, \mathbf{y}_{\mathbf{n}}\right)
$$

The process of fitting a function defined by a collection of parameters to a data set is called the discrete inverse, or parameter estimation problem as opposed to the forward problem to find $\mathbf{d}$ given $\mathbf{x}$ and $G(\mathbf{x})$. This is a familiar procedure when the determination of model parameters is accomplished using linear or polynomial regression. However there are approaches even to fitting an arbitrary function to a noisy dataset. In this work we use the conventional Maximum Likelihood Estimate method to identify the model parameters.

Using MLE we seek to know what is the probability that this data set occurred given the set of model parameters. In other words, what is the likelihood of the parameters given the data. Bayes' Theorem holds that

$$
\operatorname{prob}(X \mid D, I)=\frac{\operatorname{prob}(D \mid X, I) \times \operatorname{prob}(X \mid I)}{\operatorname{prob}(D \mid I)}
$$

where $D$ are the observations (dataset), $X$ is the vector of parameters, $I$ is general background information about the problem including the mathemat- 
ical model, and

$$
\begin{array}{ll}
\operatorname{prob}(X \mid D, I) & \text { posterior probability density function, } \\
\operatorname{prob}(D \mid X, I) & \text { likelihood function, } \\
\operatorname{prob}(X \mid I) & \text { prior probability density function, } \\
\operatorname{prob}(D \mid I) & \text { evidence. }
\end{array}
$$

The posterior probability density function $(\mathrm{PDF}) \operatorname{prob}(X \mid D, I)$ is ultimately what we wish to estimate, the prior $\operatorname{PDF} \operatorname{prob}(X \mid I)$ reflects our knowledge of the system, and the evidence $\operatorname{prob}(D \mid I)$ is the likelihood of the data based on our knowledge. We also note that since it only makes sense to compare the conditional PDFs for the same data we can ignore the denominator. We further note that the prior $\operatorname{prob}(X \mid I)$ is fixed before our observations and so can be treated as invariant to our problem. We can therefore infer that $\operatorname{prob}(X \mid D, I) \propto \operatorname{prob}(D \mid X, I)$. The MLE for the model parameters $\mathbf{x}_{\mathbf{0}}$ is given by the maximum of the posterior PDF, which is equivalent to the solution of the ODE given the parameters $\mathbf{x}$ that produces the highest probability of the observed data. Since the likelihood $\mathcal{L}(\mathbf{x})=\prod_{i}^{n} \mathcal{P}_{i}$ and the probability $\mathcal{P}$ of any single observation is less than one, then the total likelihood which is the product of a large number of probabilities is vanishingly small. The more well-behaved log-likelihood is given by

$$
\mathcal{M}=\ln (\mathcal{L})=\ln (\operatorname{prob}(D \mid X, I))=\text { const }-\frac{\chi^{2}}{2},
$$


where

$$
\chi^{2}=\sum_{i=1}^{n} \frac{\left(y_{d_{i}}-y_{G_{i}}\right)^{2}}{\sigma_{d_{i}}^{2}}
$$

is the $\chi^{2}$ goodness-of-fit, $y_{d}=y_{d}(t)$ is an observation of drop position at a point in time, $y_{G}=y_{G}(t, \mathbf{x})$ is the drop position predicted by the solution to the equation of motion at time $t$, and $\sigma_{d}$ is the standard error of the position measurement. The optimal parameter set is the one with the highest probability of observing the data (the maximum of the posterior PDF) and can be determined by maximizing the $\log$-likelihood $\mathcal{M}$ (or minimizing $\chi^{2}$ ) of the data $\mathbf{d}$ with respect to the parameter set $\mathbf{x}$. Thus, parameter estimation herein is a variety of optimization problem.

\subsubsection{Smoothing}

All optimization methods, whether explicitly or implicitly, follow gradients towards an optimum. In a parameter estimation problem, if we approximate these gradients by finite differences, then the noise manifests itself as amplification of the roughness in the hyper-response surface. Gradient-based optimizers do poorly in these situations because they tend to converge to local minima. While so called gradient free algorithms offer an improvement in this regard, speed of convergence and the quality of the MLE is improved by smoothing the objective function. This is equivalent to smoothing the underlying dataset.

Our choice of smoothing approach depends principally on the nature of the errors in the dataset. The sources of error include misalignment of the camera, error in the fiduciary length scale, perspective due to objects being 
out of the photographic plane, and various errors arising in the digitization process including the difference between the thresholded ellipse-fitted centroid and the true centroid of the non-ellipse drop centroid. Some of these errors are systematic in origin and introduce consistent biases into the data, e.g., coherent spectral sources rather than truly stochastic noise. Data smoothing does little to help systematic errors in that they are usually of lower frequency than the signal. Random errors by contrast are assumed to have a Gaussian distribution as required by the central limit theorem and are independent of the signal which we assume results from an inherently deterministic process.

We experimented with a variety of filters implemented in the scipy . signal Python SciPy [52] module on a representative set of trajectory data; these methods include 1D Gaussian convolution, Wiener, Butterworth, and SavitskyGolay filters. Qualitatively comparing these smoothing methods by handtuning filter orders and window sizes, we find that we loose too many data points in the smoothing process, that large amplitudes are overly smoothed by repeated filtering passes, or that there are significant end effects for most of these methods. A comparison of these smoothing approaches on a representative trajectory data set is shown in Figure 3.6. The Savitsky-Golay and Butterworth filters both produce fairly smooth derivatives but the small window-size needed for Butterworth filter tends to also produce noticeable end effects. The Savitsky-Golay filter essentially uses a moving-window based on local least-squares polynomial approximations. It was shown that fitting a polynomial within a moving window and then evaluating the resulting polynomial at a single point within said window is equivalent to a discrete 


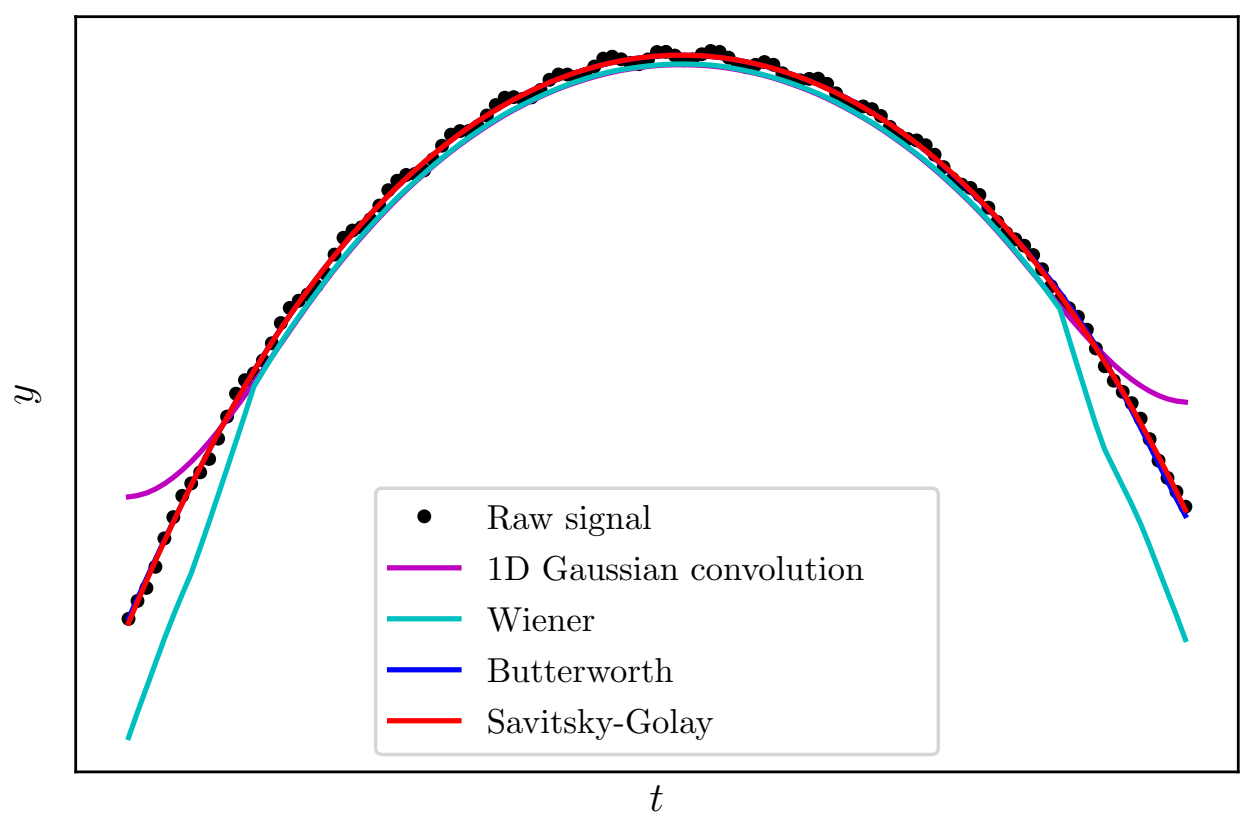

(a)

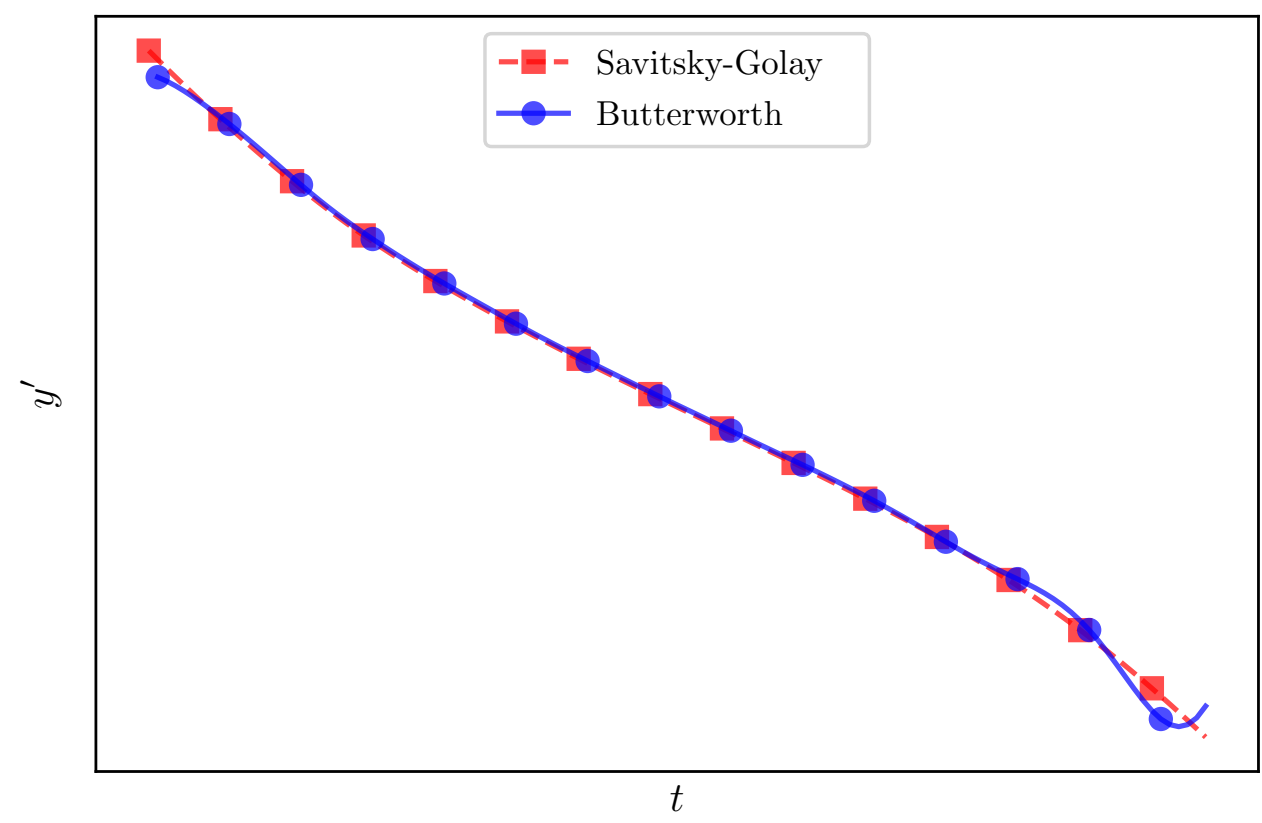

(b)

Figure 3.6: (a) Schematic noisy drop trajectory data. We see that 1D Gaussian convolution and Wiener filters suffer from significant end effects. At this scale Butterworth and Savistsky-Golay filters are nearly indistinguishable. (b) Comparing Butterworth and Savitsky-Golay filtered first derivatives of the above schematic trajectory we see that the Butterworth filter also suffers from a slight end effect. This implies that the optimization will find a different optima of the likelihood depending on which type of filter is used. 


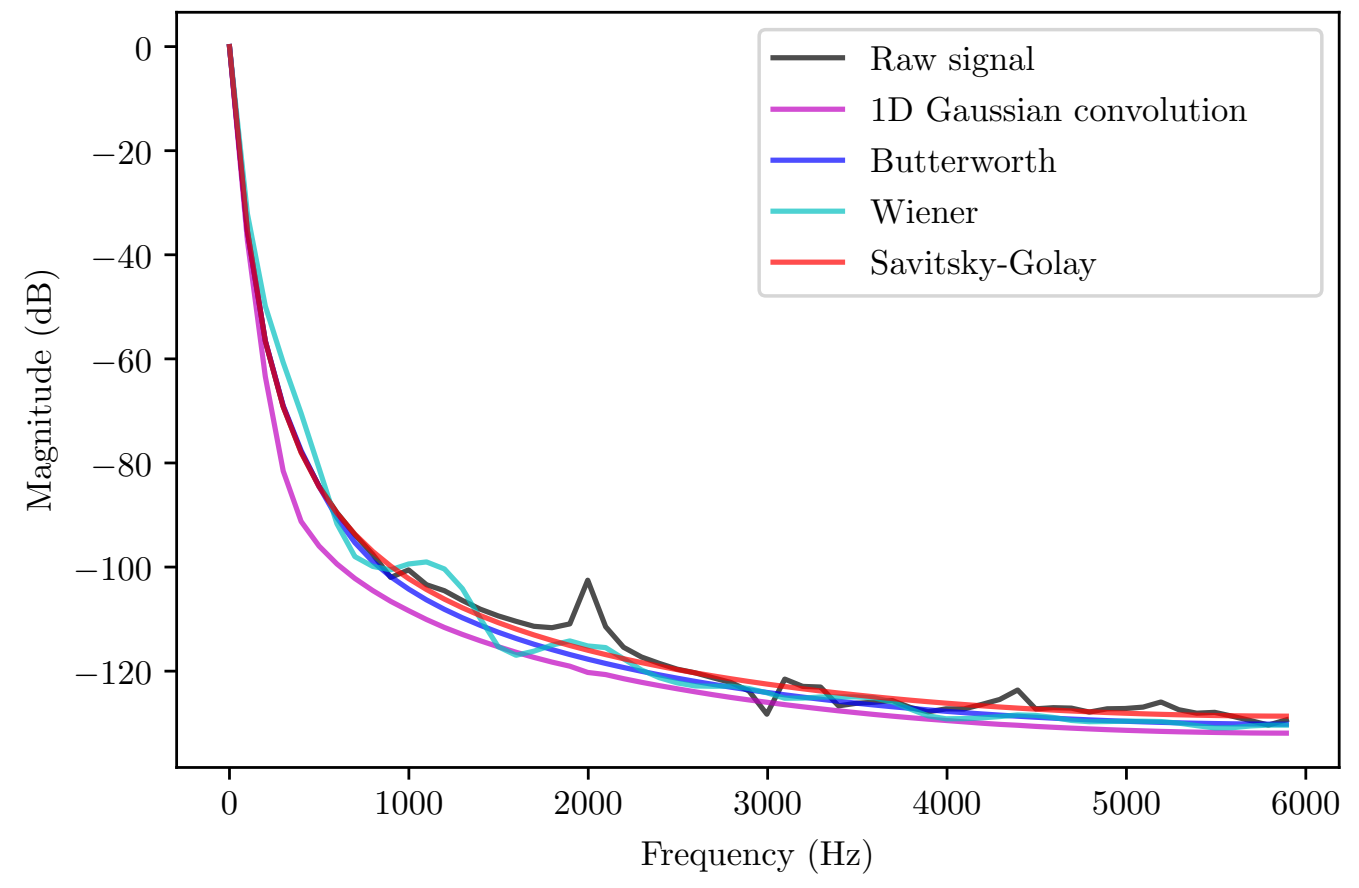

Figure 3.7: Signal power spectra with peak at $1 \mathrm{~Hz}$, which is the drop trajectory parabola itself, and smaller peaks in the $\mathrm{kHz}$ range corresponding to various noise frequencies. The Savitsky-Golay and Butterworth filters appear to have the least distortion and attenuate the noise at the $2 \mathrm{kHz}$ peak. 
convolution with a fixed impulse response [53]. A beneficial property of this kind of low-pass filter is the tendency to maintain waveform amplitudes, and so they are attractive in applications having noisy signals with sharply pointed waveforms such as ultrasound or synthetic aperture radar [54]. Because Savitsky-Golay is a Finite Impulse Response (FIR) filter it requires data points to be equally spaced. To accommodate this requirement we interpolate points between the small gaps which sometimes occur in the tracking results from image analysis. We use a moving window size slightly smaller than the length of the current bounce in a single drop jump data set. The windows are piecewise defined by partitioning the data set into a series of individual bounces. This partitioning is accomplished by slicing the dataset at minima identified after an initial rough smoothing pass using the scipy.signal.argrelextrema() function. The Savitsky-Golay polynomial order is 4 .

To understand how these filters differ it is useful to look at their frequency responses. The power spectra for these same data, obtained by Discrete Fourier Transform, are shown in Figure 3.7. We note that our signal is not truly periodic. 


\subsubsection{Optimization}

Most generally, a constrained optimization problem is stated as

minimize: $\quad f(\mathbf{x}) \quad$ objective function,

subject to:

$$
\begin{aligned}
& g_{j}(\mathbf{x}) \leq 0 \quad \text { inequality constraints, } \\
& h_{k}(\mathbf{x})=0 \quad \text { equality constraints, } \\
& \text { where } \mathbf{x}=\left\{\begin{array}{l}
x_{1} \\
x_{2} \\
\vdots \\
x_{n}
\end{array}\right. \text { design variables. }
\end{aligned}
$$

Mathematical optimization is the problem of finding minima of a function $f$. In this context the function is called the cost or objective function. The field of mathematical optimization is as old as calculus itself and the number of particular optimization techniques is correspondingly myriad. Particular techniques lend themselves well to particular types of optimization problems. The minima of the objective function $f$ is sought on a domain $A$ specified by the constraints of the problem, where the domain is usually called the feasible region. Minima of objective function $f: A \rightarrow \mathbb{R}^{m}$ are called feasible solutions. If the function $f$ is convex the feasible solution is the global minimum- otherwise additional local minima exist. The scale of the optimization problem is set ultimately by dimensionality of the objective function. Functions may not always be smooth in the sense of having continuous derivatives, and this is problematic in that optimization 
methods fundamentally rely on gradients of the objective function. Problems with anisotropic objective functions where there is strong covariance between the parameters are called ill-conditioned. In such cases the gradient vector differs significantly from the Newton direction $-\mathbf{H}^{-1} f^{\prime}(\mathbf{x})^{T}$, where $\mathbf{H}$ is the Hessian matrix. Gradient-based deterministic search algorithms tend to converge slowly for ill-conditioned problems as they take a zigzagging path determined by the local value of the gradient rather than following the Newton-direction vector towards the minimum. Numerical optimization may deal with black box functions where we do not have an explicit mathematical expression of the function we are optimizing. Black box problems are challenging because we do not have access to analytic gradients of the objective function and approximating them by finite-differences is slow and noisy. In general, noisy, black box, non-linear, non-quadratic, non-convex, constrained, ill-conditioned, high-dimensional objective functions are problematic to optimize. Unfortunately, problems of this type are the essence of parameter estimation and of our problem particularly. These difficulties often lead to the characterization of parameter estimation as an 'art' rather than a science.

The equation of motion behaves stiffly due to the large disparity in Coulombic and image charge length scales. We integrate it numerically using the odeint Scipy module. This is a shake-and-bake Python wrapper for the venerable 1982 netlib ODEPACK library double-precision lsoda integrator [55] (Livermore Solver for Ordinary Differential equations with Automatic method switching for stiff and nonstiff problems). The function switches dynamically between Adams (nonstiff) and Backwards Differentiation Formulas 
(BDF, stiff) according to the stiffness of the solution.

Our specific optimization problem is non-convex, mixed discrete-continuous black-box (noisy), and highly ill-conditioned which is essentially the worst case scenario for an optimization problem. The ill-conditioning arises due to the strong covariance between several of the model parameters, particularly $q=q\left(V_{d}, E_{0}\right)$. The non-convexity of the problem implies that there are many local minima of the objective function. While in principle a gradient-based optimizer (i.e., the quasi-Newton method of Broyden, Fletcher, Goldfarb, and Shanno (BFGS) [56]), could be used by using finite-differences to obtain approximate gradients of the $\chi^{2}$ objective function, doing so in practice is problematic because the noise-to-signal ratio of the objective function scales like $\mathcal{O}(f)$ for $d f / d t$ and $\mathcal{O}\left(f^{2}\right)$ for $d^{2} f / d t^{2}$ which will tend to cause convergence to a local minima that is only an artifact of the likelihood response surface [57]. As a further practical matter, if evaluating the objective function is computationally expensive, as in our problem, then gradient-free approaches tend to converge faster regardless [58].

We use a gradient-free direct-search Nelder-Mead [59] algorithm implemented in scipy.optimize. Nelder-Mead is relatively robust to noise and is thrifty with our computationally expensive function-calls. Nelder-Mead, sometimes called simplex-search or downhill-simplex, is a heuristic search method with no guarantee of optimal solutions. It nonetheless is a wellestablished and widely used algorithm. Nelder-Mead is based on the concept of an $N$-simplex, which generalizes a triangle into higher dimensions as a polytrope of $N+1$ vertices in $N$ dimensions. It uses only-function calls and expands or contracts the simplex according to the function values at its ver- 
tices in a way visually reminiscent (in $\mathbb{R}^{3}$ ) of the oscillations of the jumping drops themselves. In fact Nelder-Mead is sometimes also called the 'amoeba method'. Little is known about the convergence properties of the NelderMead algorithm in its classical form for non-smooth objective functions [60], except that in general it does not satisfy the properties required for convergence by other direct search algorithms: the simplex remains uniformly non-degenerate and some form of sufficient descent condition for function values at the vertices is required at each iteration. Scaling can help solve convergence problems and improve numerical stability. We precondition the optimization problem by minimizing $\ln \left(\chi^{2}\right)$ and using a naive $\sim \mathcal{O}(1)$ scaling of our constraints by their initial guesses. Here the goal is to make the problem equally sensitive to steps in any direction. Nelder-Mead is not a global optimizer, though there are variants which use sequential local searches with probabilistic restarts to improve globality, usually at a tremendous computational cost. However, Nelder-Mead behaves less locally than many gradientbased approaches. The convergence history of the parameter MLE using Nelder-Mead for a single drop jump experiment is shown in Figure 3.8. As is typical with Nelder-Mead much of the improvement in $\chi^{2}$ is realized in the first few iterations. Overall the rate of convergence is sub-linear, which is to be expected for non-linear constrained problems using an heuristic algorithm. Results of the parameter estimation are shown in Figures 3.9 and 3.10 .

\subsubsection{Identifiability}

That we are capable of fitting any arbitrary model to a dataset given sufficient degrees of freedom in our parameters is admittedly a disconcerting 


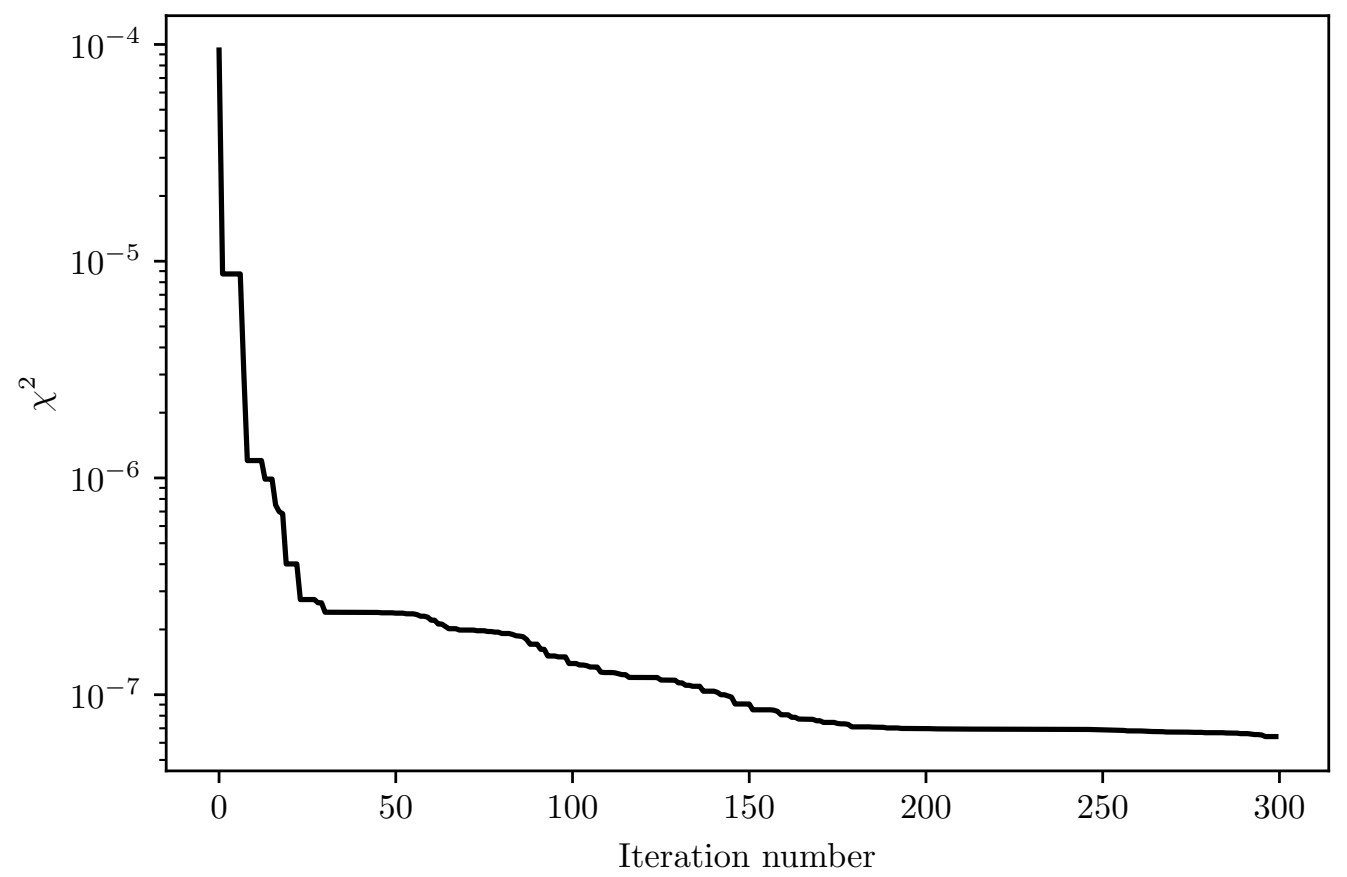

Figure 3.8: Convergence history of MLE optimization by the Nelder-Mead algorithm for a typical drop trajectory.

issue, begging the question 'given the structure of the model is it possible to uniquely estimate the unknown parameters?' This question is called the problem of identifiability. However, apart from the drop free charge the inverse model parameters are constrained by our experimental observations of them and their associated measurement uncertainties. This we hope makes the specter of an over fit model less worrisome, but does convert our unconstrained optimization problem to an constrained one which raises special difficulties of its own, which we discuss below.

The Nelder-Mead direct search method cannot be used with explicitly constrained problems. However, there are various implicit approaches to approximately solve general constrained problems using unconstrained algo- 

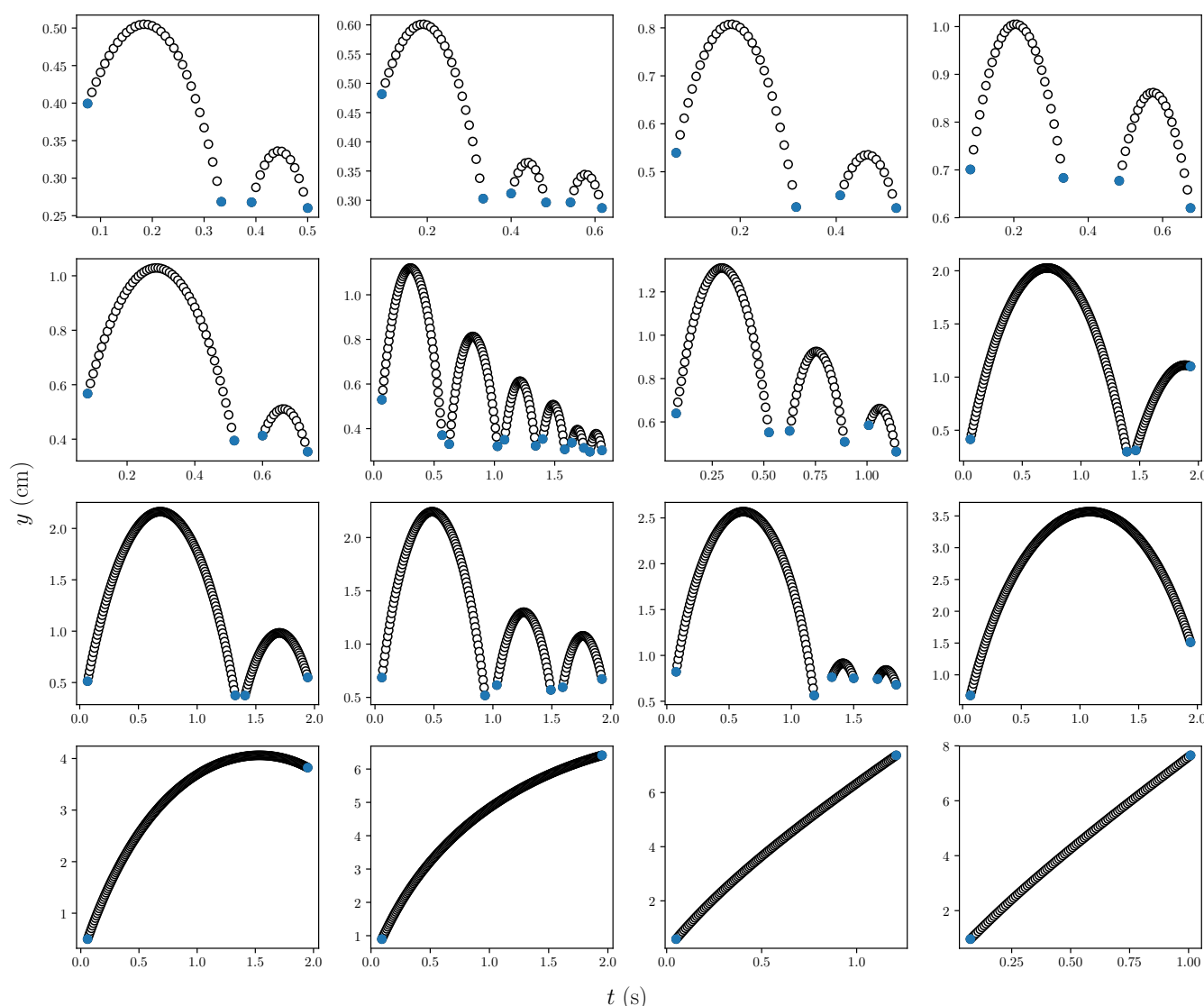

Figure 3.9: A series of filtered drop trajectories arranged by increasing apoapse. The blue dots represent either the beginning and end of the experiment, or points at which the drop is either coming into or leaving contact with the surface.

rithms. Generally, this is achieved by domain transformations or the use of penalty functions. By the addition of a penalty function which depends in some way on the values of the constraints to the objective function, we minimize a pseudo-objective function where the in-feasibility of the constraints is minimized simultaneously to the objective function.

There are various penalty function schemes. We use an exterior penalty function as a simple way of converting converting the constrained problem 


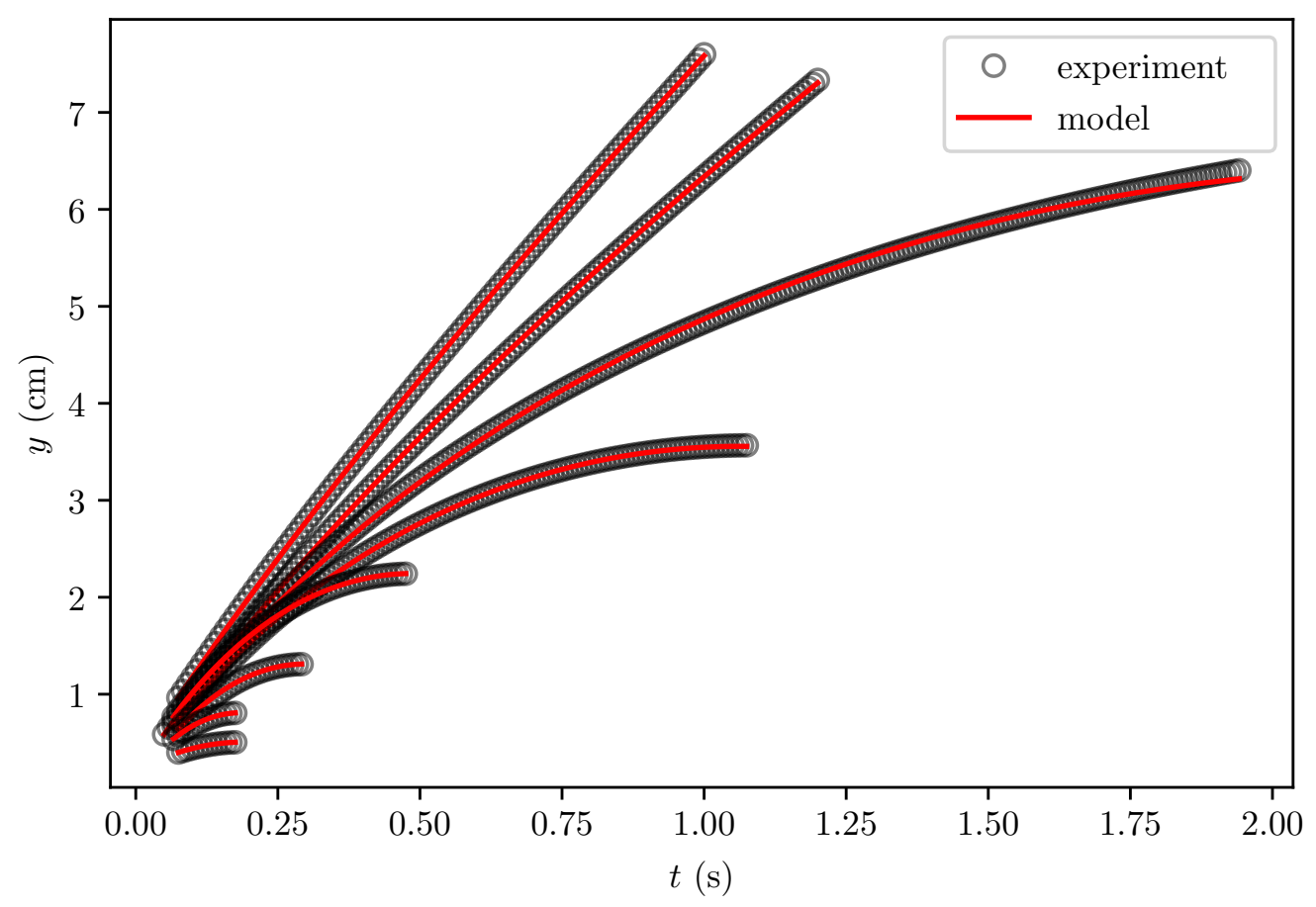

Figure 3.10: A set of drop trajectories showing the results of the parameter estimation. The trajectories are shown only up to the apoapse of the first bounce. The $(-)$ lines show the ODE solution with the given MLE parameter vector. $\chi^{2}$ goodness-of-fit varies between $1 \times 10^{-5}$ and $1 \times 10^{-8}$ with the better fit occurring typically for the drops with the lowest apoapses.

into an unconstrained one. These are especially useful in cases where the constraints are not 'hard' in the sense that they need to be precisely satisfied. General penalty functions, which are sequential unconstrained minimization techniques, reformulate the general constrained problem as the pseudo-objective function given by

$$
\phi\left(\mathbf{x}, r_{p}\right)=F(\mathbf{x})+r_{p} P(\mathbf{x})
$$


where the penalty function $P(\mathbf{x})$ is given by

$$
P(\mathbf{x})=\sum_{j=1}^{m}\left(\max \left[0, g_{j}(\mathbf{x})\right]\right)^{2}+\sum_{k=1}^{l}\left[h_{k}(\mathbf{x})\right]^{2}
$$

We see from Equation 3.1 that there is no penalty if the inequality constraints $g_{j}(\mathbf{x})$ and equality constraints $h_{k}(\mathbf{x})$ are satisfied.

As with penalty function approaches generally, the exterior penalty function has the drawback of the objective function possibly being undefined outside of the set of feasible solutions. Additionally, by naively encouraging feasibility of the solution using large values of the penalty parameter $r_{p}$ we will tend to ill-condition the unconstrained formulation of the problem, though in our implementation the preconditioning tends to make the pseudoobjective function less and less sensitive to the constraints as the likelihood is approaches a maximum. We use the measured values of $U_{0}, V_{d}$, and $E_{0}$, and the informed guess $q \approx k V_{d}^{2 / 3} E_{0}$ where the constant $k \approx 1 \times 10^{-11}$ is an initial guess for the parameter vector $\mathbf{x}$. We stop the optimization after 300 iterations rather than waiting for convergence.

We are interested in the variance and co-variance as a means to determine the quality of the parameter estimate. The $(i, j)$-th element of the matrix $\sigma(\mathbf{x}, \mathbf{y})$ is equal to the covariance $\operatorname{cov}\left(X_{i}, Y_{j}\right)$ between the $i$-th scalar component of $\mathbf{x}$ and the $j$-th scalar component of $\mathbf{y}$. Here the concept of error bars in linear correlation associated with a covariance matrix is not suitable. However, there is another approach to find the covariance matrix, given by

$$
\left[\sigma^{2}\right]_{i j}=-\left[\left(\nabla^{2} \mathcal{L}\right)^{-1}\right]_{i j}=2\left[\nabla^{2}\left(\chi^{2}\right)\right]_{i j}^{-1}=-\mathbf{H}^{-1}
$$


where $\left[\sigma^{2}\right]_{i j}$ is the covariance matrix. The issue of identifiability is especially fraught for non-linear black-box type problems were it is difficult to explicitly evaluate the Hessian. The likelihood function, and thus the posterior probability density function, are defined completely by the optimal solution $\mathbf{x}$ and the second derivative of $\mathcal{L}$ at the maximum, which corresponds to the covariance matrix. The standard errors, or marginal variances, are the square roots of the diagonal of the covariance matrix. The Hessian matrix must be negative definite for $\mathcal{L}$ to have a maximum at $\mathbf{x}_{\mathbf{0}}$. We obtain an approximate Hessian using the approach suggested in Nelder's original paper by fitting quadratics to the simplex vertices and midpoints in the vicinity of the optima [59, 61]. Thus armed with the Hessian we may use the condition number

$$
\operatorname{cond}(\mathbf{H})=\frac{\max (\operatorname{eig}(\mathbf{H}))}{\min (\operatorname{eig}(\mathbf{H}))}
$$

to make a qualitative assessment of the stability of the problem. Among our parameter estimate MLE optima we find large typical condition numbers $\sim \mathcal{O}\left(10^{27}\right)$. In fact, in many cases our Hessian is not negative definite or invertible at the minmima $\mathbf{x}_{0}$, which implies unphysical negative values of the variances. These issues indicate the problem is extremely ill-conditioned near the minima $\mathbf{x}_{\mathbf{0}}$. Given that the parameter estimates are highly co-linear, it is unsurprising that the Hessian matrix is near-singular and its inversion is either impossible or involves significant numerical error. A posteriori verification of the results is crucial to bound the identifiability of the parameter estimation problem, but this remains as future work. Several sources suggest sensitivity analysis of the parameter estimates by generation of synthetic 
Monte Carlo data sets as a means of establishing identifiability 62, 63.

\subsection{Summary}

The objectives of our experimental work are to validate the earlier scaling results by identifying values of the physical parameters which appear in the relevant dimensionless groups. To accomplish this we conduct a series of lowgravity experiments in a $2.1 \mathrm{~s}$ drop tower, where we observe the trajectories of spontaneous drop jumps and rebounds from charged dielectric superhydrophobic substrates. Our procedure to quantify these physical parameters is itemized below:

- In drop tower experiments we vary the independent variables $V_{d}$ and $\sigma$ and capture the trajectories of the drops using high-speed video.

- We produce superhydrophobic electret substrates with surface potentials $0.7-4.0 \mathrm{kV}$ and contact angles $\sim 150^{\circ}$ with approximately $25^{\circ}$ contact angle hysteresis when uncharged. The dielectrics are a lamina of 3-4 corona charged $0.4 \mathrm{~mm}$ PMMA sheets. The electric field scales with the number of dielectric lamina. The superhydrophobic layer of the electret lamina is manufactured by laser etching a $\sim 775 \mu \mathrm{m}$ deep square array of pillars into the PMMA sheet, followed by a PTFE spray deposition process.

- We solve the inverse problem to identify key parameters which we cannot measure directly in the drop tower experiments. This is accomplished by Maximum Likelihood Estimation using a constrained direct search optimization. 


\section{Chapter 4}

\section{Results}

\subsection{Parameter Estimates}

We determined the distribution of most probable experimental model variables values for a population of the drops jumped in drop tower tests shown in Figure 4.1. The dependence of charge on drop surface area $A$ is immediately evident, while the effect of electric field on drop charge is less obvious. This co-linearity is likely the source of the ill-conditioning issues in the parameter estimation process. However, assuming the main effect is the interaction between charge and electric field, a Robust Least Squares model fit $q \sim k A E_{0}$ using the Python statsmodels.formula.api.RLM() function with the nonlinear transformation $A=V_{d}^{2 / 3}$ finds that $k=5.01 \times 10^{-11} \pm 2.85 \times 10^{-11}$ $\mathrm{F} / \mathrm{m}$ with $R^{2}=0.946$. This model uses Huber's T norm, median absolute scaling, and H1 covariance estimation. A contour plot showing the estimated drop free charge as a function of $V_{d}$ and $\varphi_{s}$ is shown in Figure 4.2 .

A two-way T-test comparison of charge distributions between the drop bounce experiment and a corollary experiment with zero electric field at the time of drop deposition on the superhydrophobic surface suggests that the drop charge is primarily induced by the electric field, rather than through 


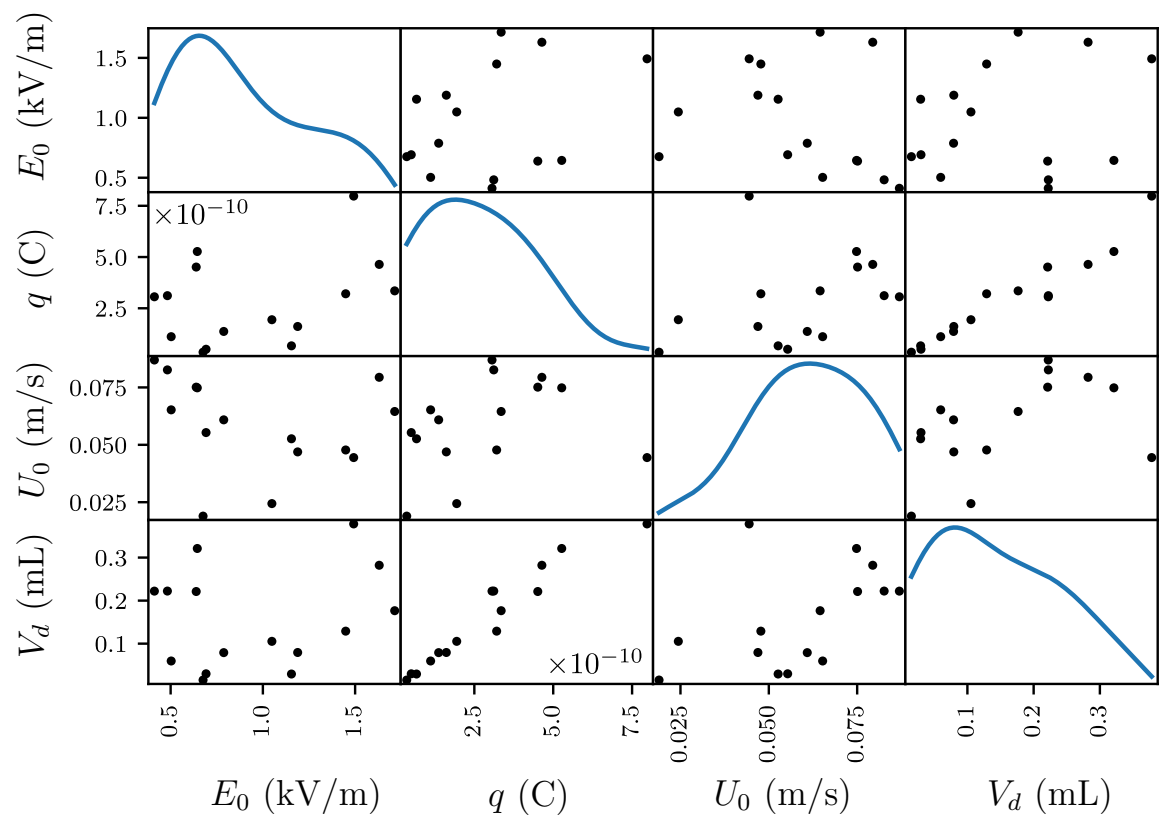

Figure 4.1: Scatter plot matrix of main parameters $E_{0}, U_{0}, V_{d}$, and $q$.

contact charging on the PTFE layer $(t=5.11, p=0.0002)$. The T-test informs us that the charge distributions are about 5 times more different from each other as they are within each other, and there is a $0.02 \%$ probability that this result occurred by chance. This corollary experiment is documented in Appendix A.

The model $q \sim k A E_{0}$ is similar to the classical solution for the surface charge of a half-spherical conductor with a field-induced dipole [36]

$$
\begin{aligned}
q & =3 \epsilon_{0} E_{0} \int_{A} \cos \theta d A \\
& =3 \pi^{1 / 3} 6\left(6 V_{d}\right)^{2 / 3} \epsilon_{0} E_{0} \int_{0}^{\pi / 2} \cos \theta d \theta \\
& =k E_{0} V_{d}^{2 / 3}
\end{aligned}
$$

with $k \approx 1.3 \times 10^{-10} \mathrm{~F} / \mathrm{m}$. This is also of similar form to the charge found 


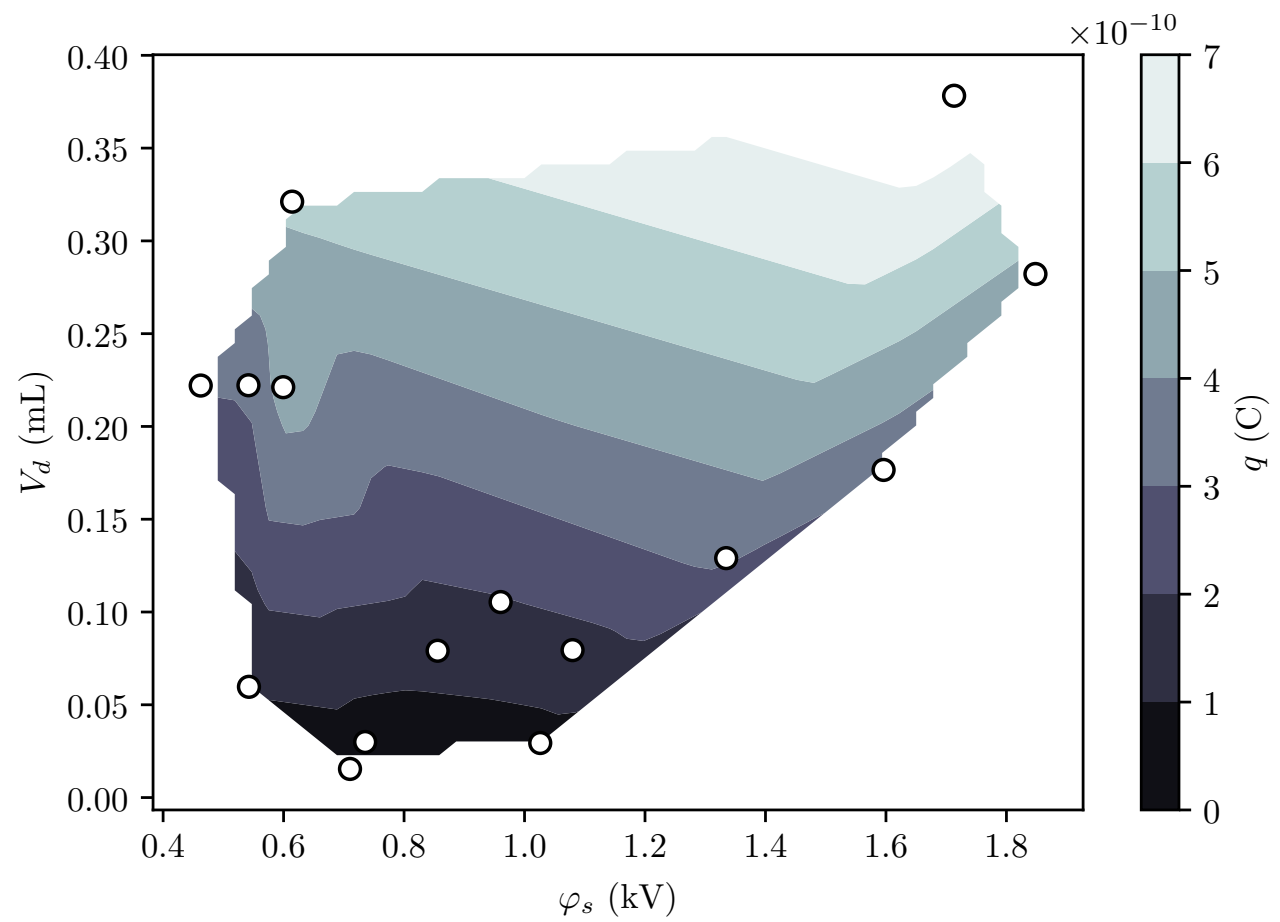

Figure 4.2: Charge $q$, as a function of the measured variables $V_{d}, \varphi_{s}$.

by Takamatsu and coauthors for drops falling from a grounded nozzle in an external electric field 64

$$
q=4 \pi \epsilon_{0} \beta E_{0} R_{d}^{2}
$$

with $\beta \approx 2.63$. Cast in the same form as Equation $4.1 k \approx 4 \pi \epsilon_{0} \beta(4 \pi / 3)^{-2 / 3} \approx$ $1.1 \times 10^{-10} \mathrm{~F} / \mathrm{m}$.

The effect of volume on jump velocity $U_{0}$ is not immediately evident in the data despite previous work having establishing this relationship [19]. This likely results from large variance in $U_{0}$ due to contact line hysteresis during the drop roll-up. Contact line losses predominate in the sub $1 \mathrm{~mL}$ volume drops which are primarily the object of this study. 


\subsection{Model Validation}

Dimensional drop apoapses shown in Figure 4.3 scale closely with $\mathbb{E u}$ as expected according to our earlier analysis. Electrostatic Euler numbers in the data set vary between $1.4 \lesssim \mathbb{E u} \lesssim 35.4$. The dielectrophoretic force plays a small role when drops have net charge in a DC field. The condition to neglect the DEP force was satisfied for all experiments in the dataset with typical values of the condition number $\kappa_{w} \epsilon_{0} K R_{d}^{2} E_{0} / q \approx \mathcal{O}\left(10^{-6}\right)$. In the nondimensional trajectories with short-time scaling shown in Figure 4.4, we see that the scaled trajectory apoapses are consistently $\mathcal{O}(1)$, with all trajectories overshooting their characteristic time scale (which predicts returns at $t^{*}=2$ at zeroth order). The fact that $\mathbb{E} u \ll 1$ is not satisfied for all tests is in violation of the Equation 2.18 asymptotic result for short times.

The predicted long-time scaled time-of-flight $t_{f}$ given by Equation 2.19 when redimensionalized by the characteristic time $t_{c}$ compares favorably to the experimental time-of-flight $t_{b}$, shown in Figure 4.5. This allows an improvement to be made to the asymptotic result of Equation 2.19 by multiplying the series by the empirical coefficient $a=1 / 0.68=1.47$. The semi-analytic time-of-flight is then given by

$$
t^{*}=2.94+1.96 \phi \mathbb{E} u+1.18 \phi^{2} \mathbb{E} u^{2}+\mathcal{O}\left(\phi^{3} \mathbb{E} u^{3}\right) .
$$

The relative magnitudes of the simulated forces felt by the entire population of drops are shown in Figure 4.6. Coulomb, image, and drag forces acting on the drops vary in typical magnitude between $\mathcal{O}\left(10^{-6}\right)-\mathcal{O}\left(10^{-4}\right) \mathrm{N}$. We see that of the drops in the experimental dataset only the two with the largest 


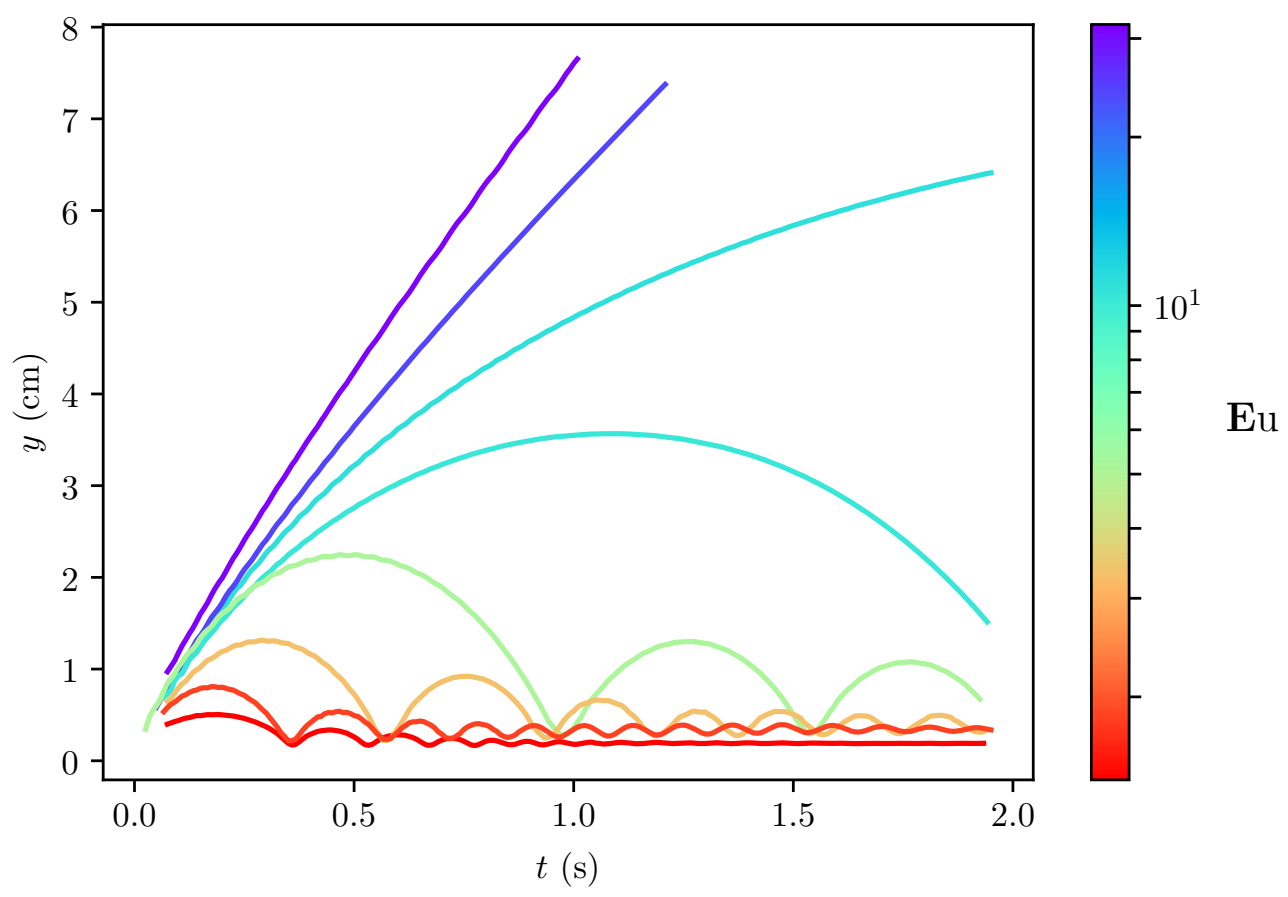

Figure 4.3: Drop trajectories as a function of $\mathbb{E u}$.

$\mathbb{E} u \sim \mathcal{O}(10)$ could appropriately be said to be in the inertial electro-viscous regime. In all other cases image forces are much stronger than drag. The image forces themselves rapidly become small compared to Coulomb forces for drops with apoapses $\max (y) \gtrsim L$. Thus, it is reasonable to claim that for intermediate drops Coulomb force scales with inertia, while drag and image forces are small. The drop with largest $\mathbb{E} u$ in our dataset failed to escape the electric field as the escape condition $\phi \mathbb{E u} / 8 \pi=0.2<1$ was unsatisfied. Equation 4.2 predicts that this drop will return to the substrate after $32 \mathrm{~s}$, a period of free-fall which is lamentably well out of reach of a drop tower. However, such an experiment could possibly be performed aboard the ISS or suborbital parabolic flights.

The covariance of $\mathbb{I g}$ with $\mathbb{E} u$ is shown in Figure 4.7. Predictably, there is 


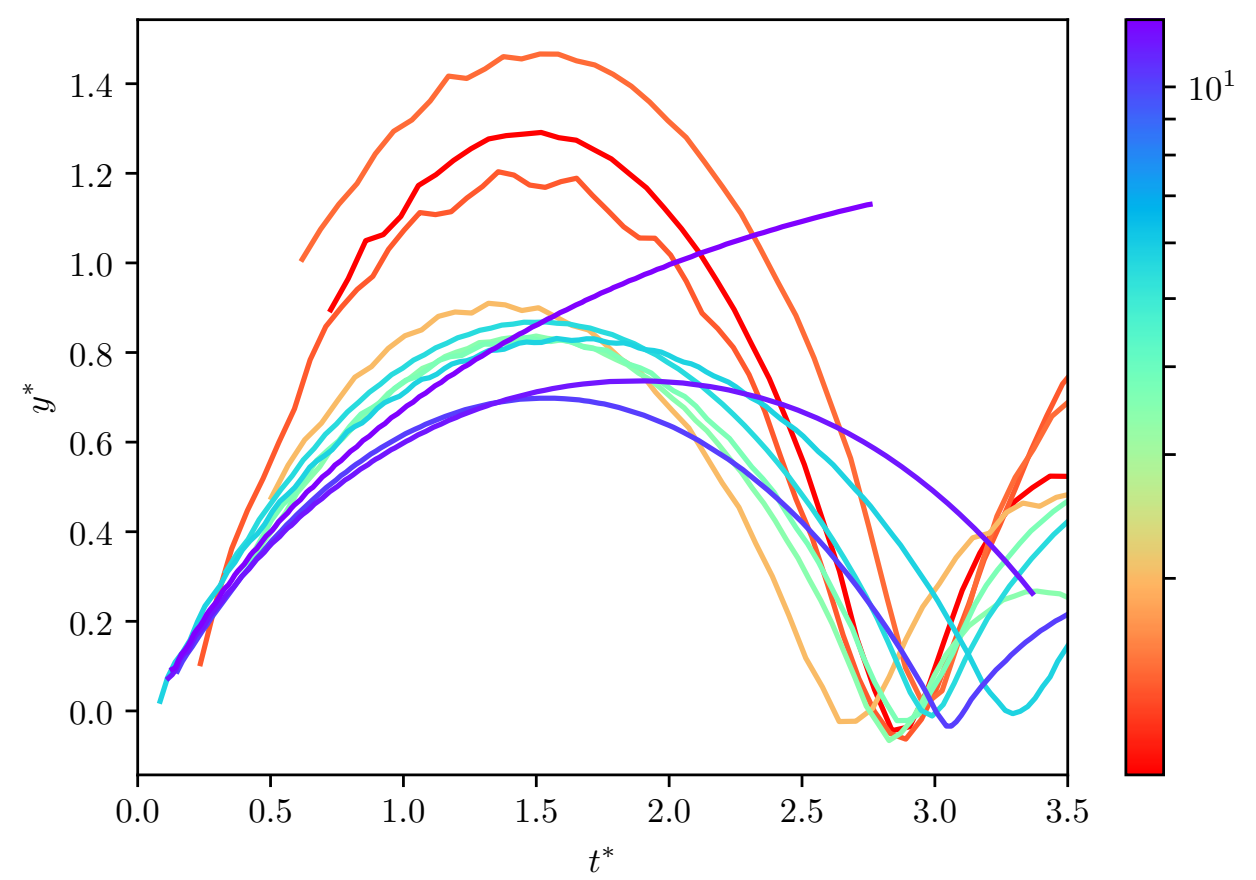

$\mathrm{Eu}$

Figure 4.4: Non-dimensional trajectories with the short-time scaling.

correlation between the dimensionless groups. We also see that $\operatorname{Ig}<1$ for all drops. Using an OLS regression, we find the model $\mathbb{I g} \sim(0.012 \pm 0.003) \mathbb{E u}+$ $(0.212 \pm 0.036)$ with $R^{2}=0.59$.

We should note the influence of systematic error on our data. We assume that drops translate purely along the central axis of the electric field. In practice, despite the improvement in surface charge density uniformity produced by corona charging, there are still local areas of especially high charge density causing radial translation and changing $E$ not as well predicted by Equation 2.9. In principle, this kind of error should become small for drops that are far enough away from the charge distribution where the geometry of the charge distribution vanishes and the electric field looks like that due to a point charge. However, for low $\mathbb{E} u$ drops this error relatively increases. The 


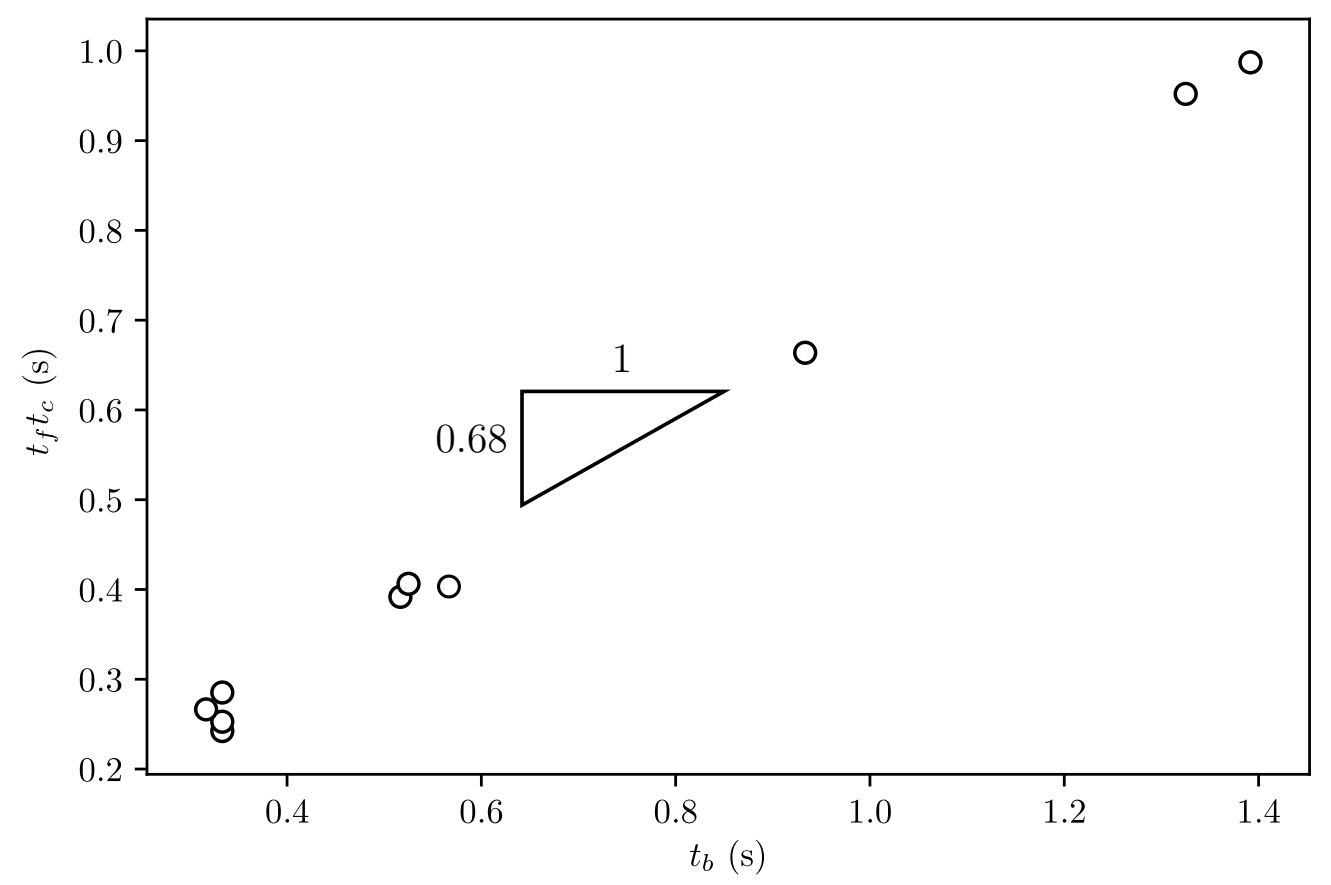

Figure 4.5: Dimensional time-of-flight asymptotic estimates $t_{c} t_{f}$ compared with experimental time-of-flight $t_{b}$.

primary sources of random error are the effect of contact line hysteresis on the drop initial velocity and of the variance in the MLE parameter estimates.

\subsection{Suggestions for Future Directions}

In $1-g_{0}$, for Weber numbers $\mathbb{W e} \equiv \rho U^{2} R_{d} / \gamma>0.4$, impact rebound behavior on a superhydrophobic surface can be strongly influenced by damping from contact line hysteresis. For the low Bond and Ohnesorge numbers occurring in free-fall, the drop impact dynamics additionally include electrohydrodynamic surface wettability effects. To date there has been little work in general on drop impacts outside of two regimes: (1) very low $\mathbb{R e}$ viscous drop spreading driven by capillary forces at the contact line and (2) impacts at 'high' 


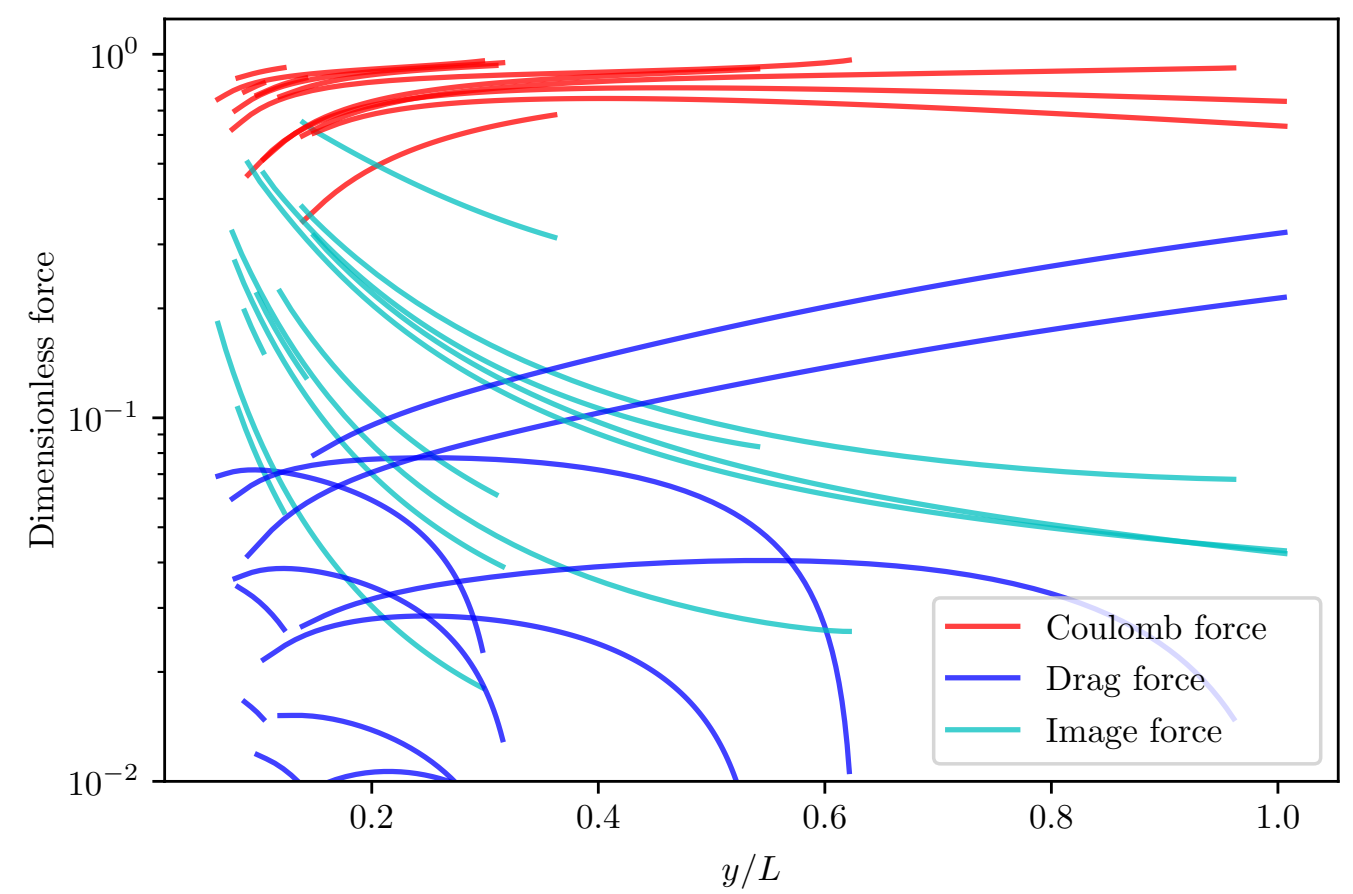

Figure 4.6: Simulated forces acting on the drop up to the first apoapse.

Weber numbers. Models for dynamic contact lines in general remain controversial, even for ordinary spreading of liquids, despite decades of work in the area.

If we naively neglect the contact line dynamics, the dimensionless groups for isothermal drop impacts are the Ohnesorge number $\mathbb{O} \mathrm{h} \equiv \mu / \sqrt{\rho \gamma R_{d}}$, the Weber number $\mathbb{W e}$, and Bond number $\mathbb{B}$. The Weber number scales the driving force of drop spreading. In the well-studied case of high $\mathbb{W}$ impacts the drop bulk is driven radially outward by the impact induced pressure gradient, whereas in the case of small $\mathbb{W}$ e wetting impacts the liquid is driven outwards by capillary force. The Ohnesorge number, by contrast, is a measure of viscous effects in an inertial-capillary flow and scales the force that resists spreading. Previous empirical work for low $\mathbb{O}$ h and low $\mathbb{W}$ impacts in- 


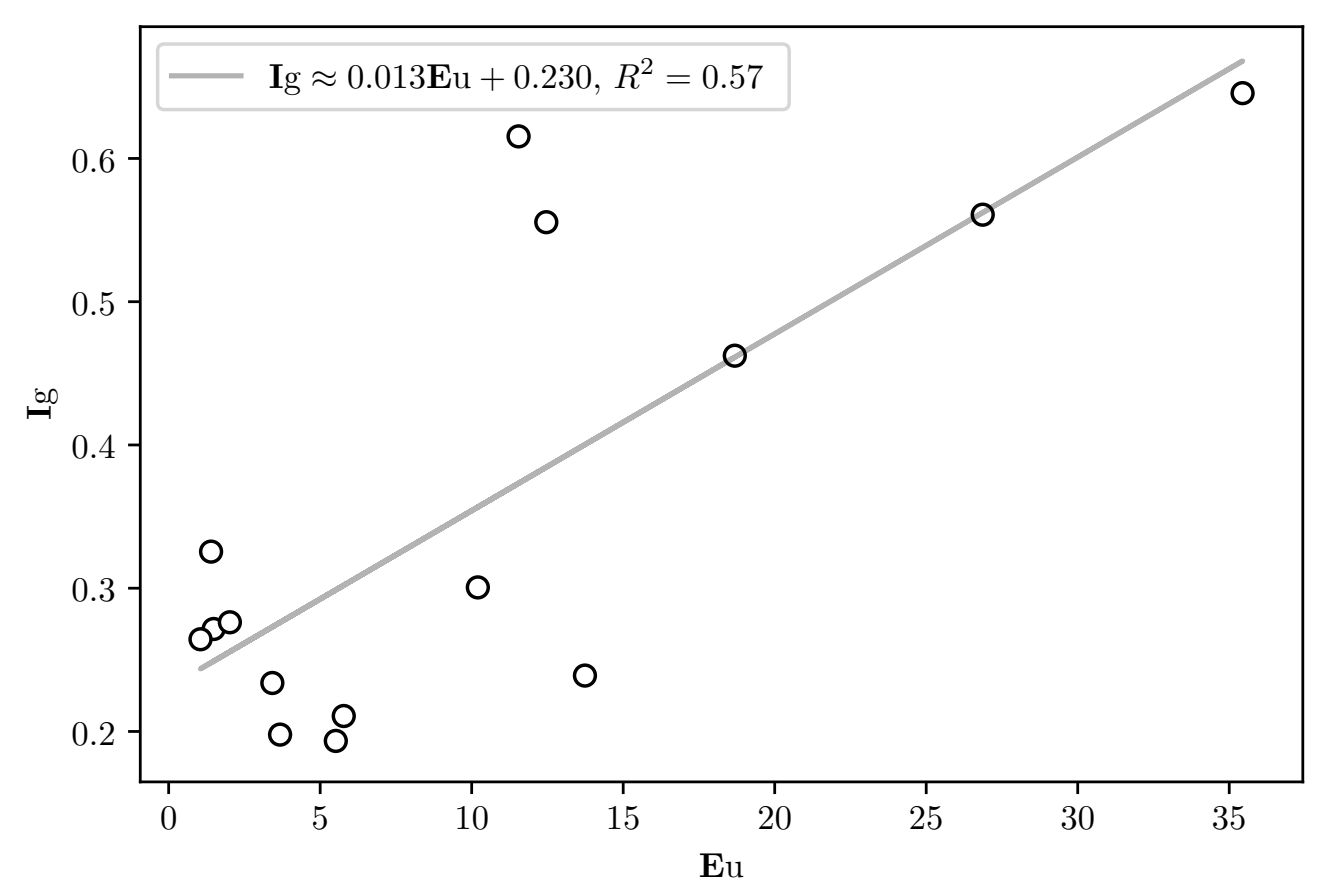

Figure 4.7: Experimental covariance of $\mathbb{E} u$ and $\mathbb{I g}$.

cludes that of Schiaffino and Sonin [65] with wetting and non-wetting impacts of molten metal drops on cold surfaces. Moláček and Bush [66], Gopinath and Koch [67], and Okamura [68] have all developed analytical models of drop impacts at low $\mathbb{W}$ e and low $\mathbb{B}$ o. These works show increasing dimensionless contact time $\tau$ as Weber number decreases, in opposition to the results of Richard and Quéré [20] which show experimentally that the dimensionless contact time is approximately a constant $\tau \approx 2.6\left(\rho R_{d}^{3} / \sigma\right)^{1 / 2}$ with respect to $\mathbb{W}$ e at large $\mathbb{W}$ e. Moláček's work also shows that impact coefficients of restitution $C_{r}$ depend non-linearly on $\mathbb{B}$ o. To date little experimental work has been performed on low $\mathbb{B}$ o impacts as well. An exception is provided by Duvivier et al. [69] who studied regimes of aqueous ferrofluid drop impacts on superhydrophobic substrates under the influence of an external magnetic 
field. In this case the magnetic body force acts as an ersatz gravity.

When the electro-drop bounce occurs, if the drop has enough time to return a non-wetting impact occurs on the charged substrate usually followed by rebound. We observe average drop impact $\mathbb{O} \mathrm{h} \approx 2.18 \pm 0.36$ and $\mathbb{W e} \approx 0.28 \pm 0.22$. Thus impact velocity plays little role in the spreading dynamics of the bounces and viscous effects are important but do not dominate inertia. Notably we observe underdamped oscillations of drop interfaces during impact. Access to such relatively low $\mathbb{O}$ h and low $\mathbb{W}$ e drop impacts enabled by the low-gravity environment raises an interesting possibilities for new work on the basic science of drop impacts. This work also intersects the burgeoning field which spans the intersection of electrowetting on patterned surfaces and drop impacts. Various authors [70, 71] have suggested that a wetting transition called Fakir impalement can occur during impacts on patterned hydrophobic surfaces if a certain critical pressure $p_{c} \sim \gamma h / l$ is exceeded, where $h / l$ is the pillar aspect ratio of the patterned surface. This pressure can result from fluid inertia in a high $\mathbb{W}$ e impact regime, or can result from an electrostatic pressure due to an external electric field. The latter case is responsible for the irreversiblity that notoriously plagues static EWOD experiments. There is hope that additional work in this area could produce engineered surfaces that are tuneably wetting under drop impacts by leveraging electrostatic forces.

Our preliminary results showing the influence of $\mathbb{W} e$ and electrostatic Bond number $\mathbb{B o}_{e} \equiv \epsilon E_{0}^{2} R_{d} / \gamma$ on drop impact dimensionless contact time $\tau$ and coefficient of restitution $C_{r}$ at $\mathbb{O} \mathrm{h} \approx 2.2$ are shown in Figures 4.8 and 4.9 . 


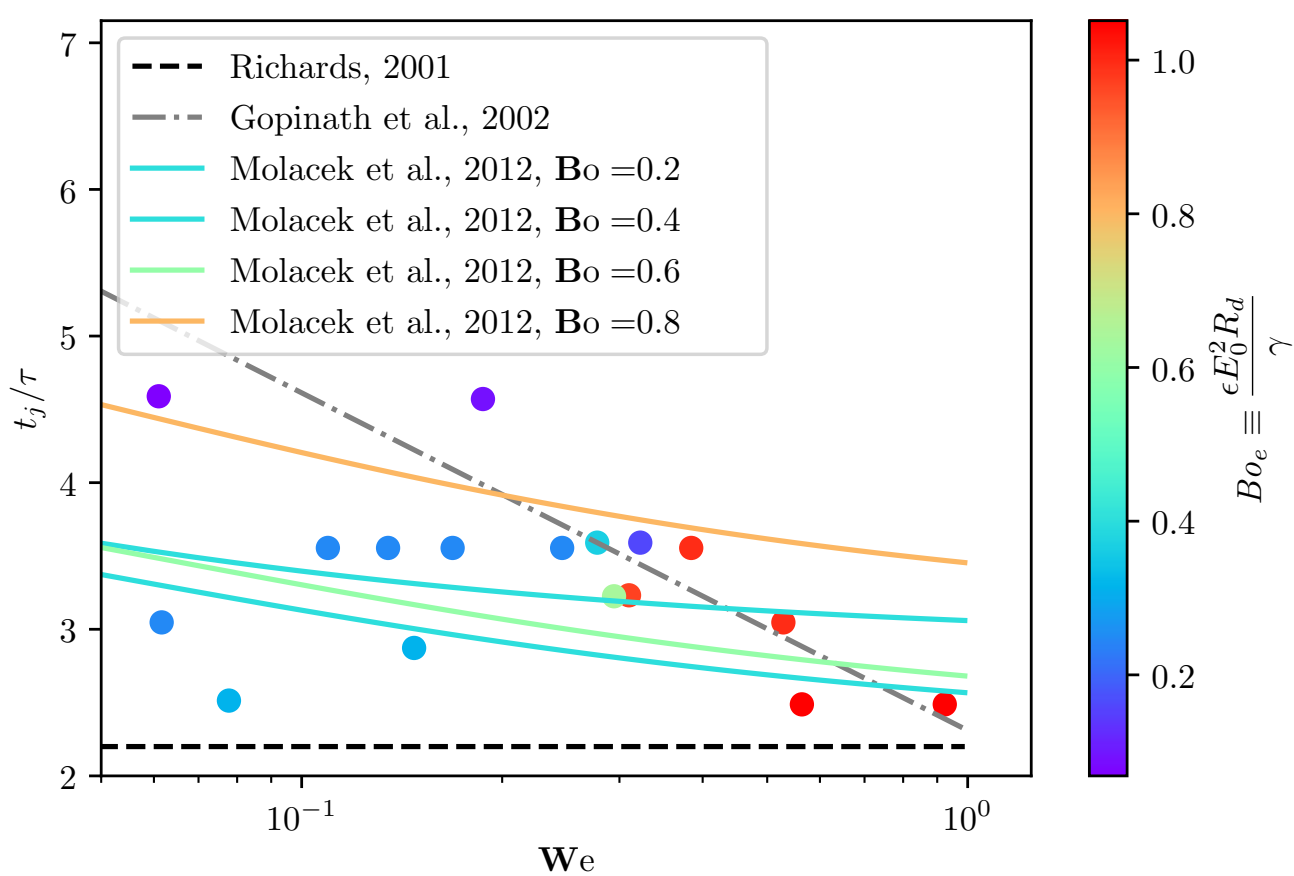

Figure 4.8: Dimensionless contact time $t_{j} / \tau$ compared with impact $\mathbb{W}$ e and $\mathbb{B o}_{e}$.

\subsection{Conclusion}

This work might have stopped early had we maintained our focus on spontaneous drop jump with the key result being simply a means of mitigation for this troublesome electro-bounce phenomenon. In some sense this is quite easy to do as there are a plethora of commercially available static charge neutralization tools aimed at ESD control applications. In a low-gravity setting, if we wish to rid ourselves of these electrostatic phenomenon we need simply to neutralize dielectric surfaces in the vicinity of our controlled environment. A recipe for accomplishing this is provided in Appendix B. Of course there are limitations to this approach. For instance, if the work involves surface chemistry on the surface requiring neutralization, depositing 


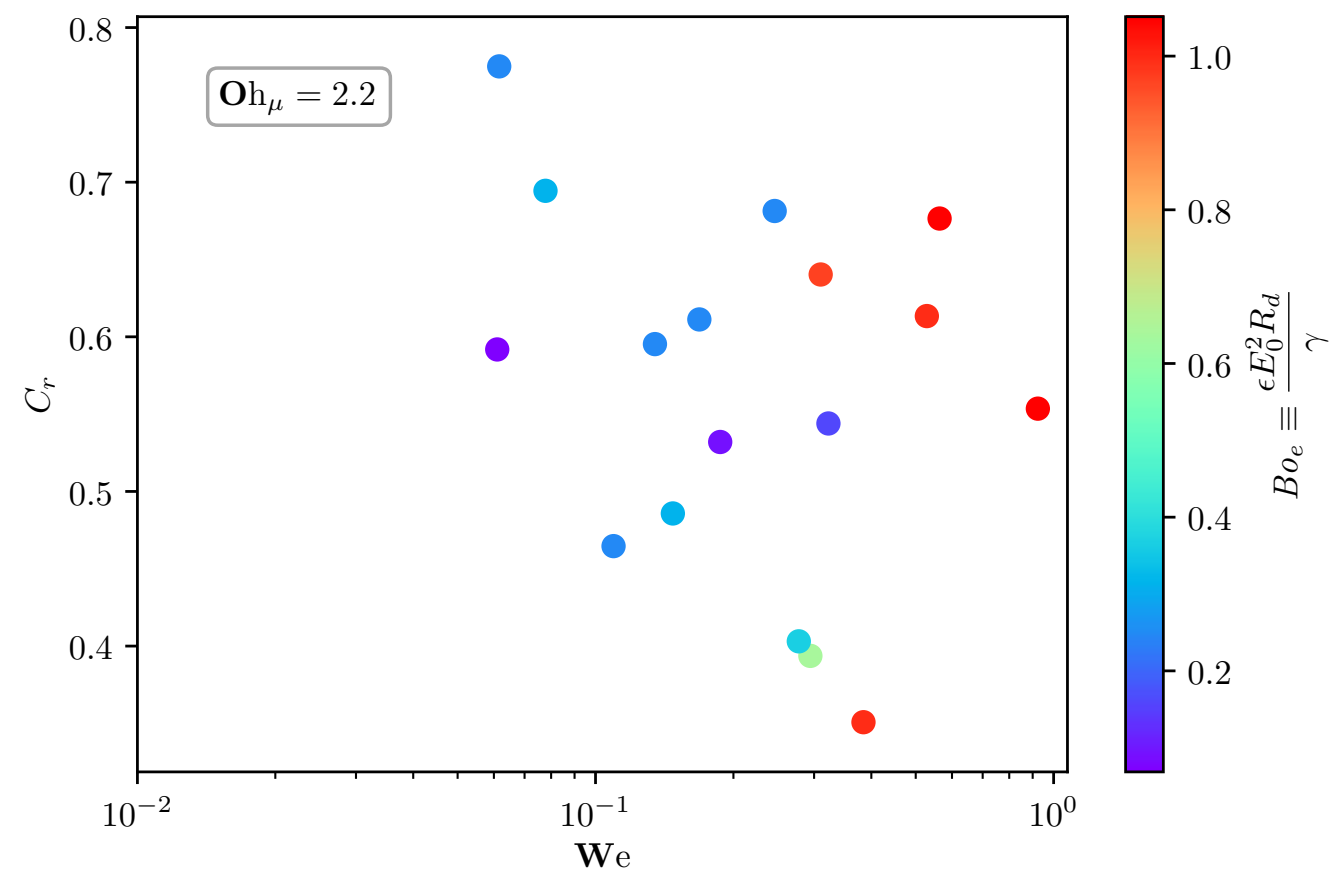

Figure 4.9: Impact coefficient of restitution $C_{r}$ compared with impact $\mathbb{W e}$ and $\mathbb{B o}_{e}$.

a layer of ions will possibly change experimental results. Additionally, there are cases where we do not control the environment for practical reasons. For example, aboard the ISS crew clothing can easily become electrostatically charged just as clothing does on Earth. If this charge distribution is in the vicinity of Rayleigh-Plateau breakup of a fluid, as might happen when squirting water from a drink bag, then electrophoresis of the drops will occur after the fashion of this work.

Our experimental results have shown that the simple 1-dimensional model is reasonably accurate at predicting trajectories of charged drops. We conclude this thesis by turning the theory to a simple space systems application in a worked example. Let us say we desire to capture drops in a pre-stage 
to the compressor of a Rankine cycle space nuclear power system. We must actively capture the drops because we require a single-phase saturated vapor at the compressor inlet but do not wish to superheat or interrupt the flow. The drops are polydisperse and thus have a distribution of masses $m$ and velocities $U_{0}$. The working fluid is a mixture of lithium and cesium. The lithium droplets have high surface tension even at the high temperatures encountered in this power cycle, and an insulated electrode can possibly be made hydrophobic by means of a high temperature coating such that the drops rebound rigidly without wetting the surface of the precipitator. The lithium drops can also be treated as ideal conductors, and we assume the droplet velocity vector is normal to the precipitator electrode as in our work. The simple 1-dimensional theory of this thesis assumes the electric field due to a square charged dielectric, but other electric fields and electrode geometries are possible or desirable for the designer. The scaling used in this work will oftentimes remain valid as electric fields for numerous geometries contain the same near-field constant and far-field $1 / y^{2}$ limits. In other cases, particularly cases with more complicated geometries, the electric field will need to be determined analytically or numerically. From the form of the electrostatic Euler number

$$
\mathbb{E} \mathrm{u}=\frac{m U_{0}^{2}}{q E_{0} R_{d}}
$$

we note that this dimensionless group can also be thought of as a ratio of energies: the kinetic energy $m U_{0}^{2}$ in the numerator and the electrostatic potential energy $q E_{0} R_{d}$ in the denominator. If the drops enter the system at distance $H$ from the surface then the electrostatic potential energy is better 
described by $q E_{0} H$ if the electric field is assumed to behave like $1 / y^{2}$. We also note that although the velocity $U=U(t, y)$ and electric field $E=E(t, y)$ are variables, if the system is conservative this ratio of energies remains constant. If an engineering requirement is to capture $95 \%$ the drops entering the compressor to mitigate damage to the turbine blades we can perform an initial sizing of an electrostatic drop precipitator using the scaling approach of this thesis. Let us say that two standard deviations of the drop population enter the precipitator with velocity $U_{0}>20 \mathrm{~cm} / \mathrm{s}$ and average radius $R_{d}=1$ $\mathrm{mm}$. The drops enter the system at $H=4 \mathrm{~cm}$, and the design constraints for the electrode are such that $E_{0}=3 \mathrm{kV} / \mathrm{m}$ and that the electrode is $10 \mathrm{~cm}$ square. To satisfy Equation 2.20 requires

$$
\frac{q}{m}=\frac{4 \pi R_{d}^{2} U_{0}^{2}}{L^{2} E_{0} H}
$$

From the above we find that to capture these drops a charge-to-mass ratio $q / m=4 \times 10^{-5} \mathrm{C} / \mathrm{kg}$ is required. This is one order of magnitude greater than the charge-to-mass ratios observed in this work, but can be achieved by a shielded charging electrode near an orifice in a way similar to an inkjet printer, or possibly by discharge, electron beam, or ionizing radiation emission. Though this example is highly contrived and departs far from the experiment reported here the results remain applicable because of the fundamental similarity in the underlying physics. 
Appendices 


\section{Appendix A}

\section{Parallel Plate Method}

By the earlier scaling we presuppose the source of the drop bouncing behavior to be primarily Coulombic in origin (as opposed to dielectrophoretic), thus the drop must have some free charge in addition to the charge induced by the electric field. To determine whether this free charge arises due to contact charge or field-induction concurrent methodologies were used. In a corollary set of experiments we determine the drop free charge by observation of the deflection of the drops in the region of a known uniform field in a fashion inspired by Millikan's famous experiment to determine the fundamental charge of the electron.

Drops are jumped in free-fall from a superhydrophobic substrate placed between the plates of a parallel plate capacitor of known uniform electric field. The surface charge of the substrate is neutralized using the Ptec IN5120 ion source. Since the drop initial velocity $U_{0}$ is parallel to the electric field, the drops are inertial in the direction of the electric force, and neglecting the effect of image charges mirrored across the conductors, we can determine the magnitude of the drop charge by a balance of Coulombic force and inertia given by the equation of motion 


$$
y^{\prime}(t)=q \mathbf{E} .
$$

Since the drag is negligible in the inertial limit we can find the charge $q$ by fitting a second-order least squares polynomial to the measured drop positions. We equate the $t^{2}$ term to the constant acceleration and divide by the known constant magnitude of the electric field.

A 200-880 VAC source with a full wave bridge rectifier circuit is used in the experiment to measure drop charge. measurements show that the highvoltage source circuit AC component of the signal is appropriately small (13 $\mathrm{mV}$ at $35 \mathrm{kHz}$ ). The no-load current is determined to be a relatively low $80 \mu \mathrm{A}$. The high-voltage source terminals are led to two parallel polished 150x150 mm aluminum plate electrodes. The electrodes are mounted on an insulated 80/20 extruded aluminum rail for ease of adjustment. All drop charge experiments are conducted with an electrode spacing of $28.30 \mathrm{~mm}$. With this spacing the calibrated electric field between the plates is $\mathbf{E} \approx$ $35 \mathrm{kV} / \mathrm{m}$. The electrodes are electrically isolated from the drop rig by two alternating layers of $4 \mathrm{~mm}$ thick PMMA sheet and Kapton tape. Potential across the plates is measured periodically with a load-impedance corrected multimeter to account for battery depletion. The typical capacitor rise time of the plates is measured to be $1.4 \mathrm{~s}$, thus to make the most economical use of the brief window a low-gravity a weighted spring-loaded switch is set by hand prior to the drop to close the high-voltage circuit, but which passively safes the system at the resumption of $1-g_{0}$ conditions in the tower. We suppose the drop free charge, if it is indeed arises by contact with PTFE, to be some 
function of the drop volume and the residence time on the superhydrophobic surface. However, sweeping though drop volumes over a series of drop tower experiments we find little correlation between drop volume and free drop charge.

A brief screening experiment was conducted which alternated the polarity of the field by switching the positive and negative terminal leads between plates. Qualitative observations of drop electrode preference seem to indicate that the assumption of small polarization stress was well founded. Following this a orthogonal array $3^{2}$ factorial design experiment with two replicates was conducted to test the effect of varying drop volume and surface stay time on free charge at the time of jumping. It was hypothesized that free charge would increase for levels of both factors. ANOVA analysis using pandas of the linear multiple regression model $q \sim a * V_{d}+b * t_{s}$, where $a, b$ are model fitting coefficients, for the data set indicates that neither drop volume $V_{d}$ $(p=0.178)$, nor surface stay time $t_{s}(p=0.939)$ is significant at the $95 \%$ confidence level. The overall model F-statistics (1.001 in 2 and 14 degrees of freedom), and coefficients of determination $\left(R^{2}=0.125\right)$ indicate that the linear model neither fits the data particularly well, nor does it offer an improvement over the mean model. The mean charge was thus determined to be positive $5.3 \times 10^{-12} \mathrm{C}$, with a standard deviation of $4.7 \times 10^{-12} \mathrm{C}$. 


\section{Appendix B}

\section{Charge Mitigation}

When neutralization of static charge on the superhydrophobic surfaces used in this work is required we use the following procedure:

1. Surface potential is quickly estimated using a Simco-Ion FMX-004 electrostatic fieldmeter. This measurement is only quantitative for flat dielectric surfaces larger then several cm square, but can be used qualitatively for other geometries. This sensor should be positioned about $2.5 \mathrm{~cm}$ normal to the surface in question. For quantitative measurements the sensor conductive shell should be connected to ground, and the dielectric substrate should rest on conductive backplane. In any case the surface potential measurement should establish whether there is a need for charge neutralization.

2. A Ptec IN5120 ion fan is positioned about $10 \mathrm{~cm}$ away from the surface. The ion fan issues a balanced steam of ions which will screen the net surface charge densities of the dielectric. The surface potential is reduced to the ion source bias (on the order of $\pm 10 \mathrm{~V}$ ) within 2 to 10 s. All faces of the dielectric should be discharged under the fan, as for materials with low dielectric constants, net charge distributions will set 
up significant electric fields that permeate the dielectric regardless of their position. In other words the bulk of the dielectric does little to shield the electric field.

3. We verify that the problematic charge has been duly compensated by the ion stream by remeasuring the surface potential with the fieldmeter. 


\section{Bibliography}

${ }^{1}$ B. J. Motil, J. H. Agui, and F. P. Chiaramonte, "Priorities for microgravity fluid physics research and an overview of gravity-dependent complex fluids research", (2012).

${ }^{2}$ D. A. Petrash and E. W. Otto, "Controlling the liquid-vapor interface under weightlessness", AIAA J 2, 56 (1964).

${ }^{3}$ R. M. Jenson, A. P. Wollman, M. M. Weislogel, L. Sharp, R. Green, P. J. Canfield, J. Klatte, and M. E. Dreyer, "Passive phase separation of microgravity bubbly flows using conduit geometry", International Journal of Multiphase Flow 65, 68-81 (2014).

${ }^{4}$ L. J. P. Hastings, "Saturn v/s-IVB stage low gravity fluid mechanics problems and saturn IB liquid hydrogen orbital experiment to verify propellant ullaging and orbital venting system", June 1, 1965.

${ }^{5} \mathrm{~J}$. B. Blackmon, "Collection of liquid propellants in zero gravity with electric fields", Journal of Spacecraft and Rockets 2, 391-398 (1965).

${ }^{6}$ T. J. Snyder, J. B. Schneider, and J. N. Chung, "Dielectrophoresis with application to boiling heat transfer in microgravity. i. numerical analysis", Journal of Applied Physics 89, 4076-4083 (2001).

${ }^{7}$ P. Di Marco, W. Grassi, G. Memoli, T. Takamasa, A. Tomiyama, and

S. Hosokawa, "Influence of electric field on single gas-bubble growth and detachment in microgravity", International Journal of Multiphase Flow 29, $559-578$ (2003).

${ }^{8}$ P. D. Marco, "The use of electric force as a replacement of buoyancy in two-phase flow", Microgravity Science and Technology 24, 215-228 (2012).

${ }^{9}$ T. B. Jones, "Electrohydrodynamic heat pipes", International Journal of Heat and Mass Transfer 16, 1045-1048 (1973).

${ }^{10} \mathrm{M}$. Hurwitz, "Electrohydrodynamic propellant management systems for cryogenic upper stages", in 3rd annual meeting, 0 vols., Annual Meeting (American Institute of Aeronautics and Astronautics, Nov. 29, 1966). 
${ }^{11}$ L. R. Koval and P. G. Bhuta, "Dynamics of a cryogenic liquid in an electric field", in Advances in cryogenic engineering: proceedings of the 1966 cryogenic engineering conference university of colorado engineering research center and cryogenics division NBS institute for materials research boulder, colorado june 13-15, 1966, edited by K. D. Timmerhaus (Springer US, Boston, MA, 1967), pp. 92-102.

${ }^{12}$ R. G. Fax, M. Hurwitz, and J. R. Melcher, "Dielectrophoretic liquid expulsion.", Journal of Spacecraft and Rockets 6, 961-967 (1969).

${ }^{13}$ J. E. Boretz, "Orbital refueling techniques", Journal of Spacecraft and Rockets 7, 513-522 (1970).

${ }^{14} \mathrm{D}$. Petrash and R. Raco, "Use of an electric field to attain a zero-gravity liquid-vapor interface configuration under one-gravity", (1968).

${ }^{15}$ M. M. Hurwitz, Dielectrophoretic control of propellant slosh in low gravity final report, 5 jan. 1966 - 31 mar. 1968 (Mar. 31, 1968).

${ }^{16}$ S. G. P. Berenyi, Dielectrophoretic baffling to control vapor ingestion in weightlessness (July 1, 1970).

${ }^{17}$ I. M. Kirko, E. I. Dobychin, and V. I. Popov, "Phenomenon of the capillary "ball game" under the condition of weightlessness", Soviet Physics Doklady 15, $442(1970)$.

${ }^{18}$ A. Wollman, M. Weislogel, B. Wiles, D. Pettit, and T. Snyder, "More investigations in capillary fluidics using a drop tower", Experiments in Fluids $\mathbf{5 7 ,} 57$ (2016).

${ }^{19}$ B. Attari, M. Weislogel, A. Wollman, Y. Chen, and T. Snyder, "Puddle jumping: spontaneous ejection of large liquid droplets from hydrophobic surfaces during drop tower tests", Physics of Fluids 28, 102104 (2016).

${ }^{20}$ D. Richard, C. Clanet, and D. Quéré, "Surface phenomena: contact time of a bouncing drop", Nature 417, 811 (2002).

${ }^{21}$ I. Langmuir, "Surface electrification due to the recession of aqueous solutions from hydrophobic surfaces", Journal of the American Chemical Society 60, 1190-1194 (1938).

${ }^{22}$ K. Yatsuzuka, Y. Mizuno, and K. Asano, "Electrification phenomena of pure water droplets dripping and sliding on a polymer surface", Journal of Electrostatics 32, 157-171 (1994).

${ }^{23}$ J. K. Beattie, "The intrinsic charge on hydrophobic microfluidic substrates", Lab on a Chip 6, 1409-1411 (2006).

${ }^{24}$ S. Strazdaite, J. Versluis, and H. J. Bakker, "Water orientation at hydrophobic interfaces", The Journal of Chemical Physics 143, 084708 (2015). 
${ }^{25}$ Pettit Donald, "Flow induced charging of liquids in reduced gravity", Engineering, Construction, and Operations in Space V, Proceedings 10.1061/ 40177(207)75.

${ }^{26}$ D. Stevenson, "Electrostatic model applied to ISS charged water droplet experiment", in (June 16, 2015).

${ }^{27} \mathrm{~S}$. A. Czepiela, The charging of composites in the space environment (Aug. 1, 1997).

${ }^{28} \mathrm{~W}$. Unterberg, "Zero gravity problems in space powerplants: a status survey", ARS Journal 32, 862-872 (1962).

${ }^{29}$ K. A. White, Liquid droplet radiator development status (Jan. 1, 1987).

${ }^{30}$ D. Choi, H. Lee, D. J. Im, I. S. Kang, G. Lim, D. S. Kim, and K. H. Kang, "Spontaneous electrical charging of droplets by conventional pipetting", Scientific Reports 3, 2037 (2013).

${ }^{31}$ F. F. Abraham, "Functional dependence of drag coefficient of a sphere on reynolds number", The Physics of Fluids 13, 2194-2195 (1970).

${ }^{32}$ D. A. Saville, "Electrohydrodynamics: the taylor-melcher leaky dielectric model", Annual Review of Fluid Mechanics 29, 27-64 (1997).

${ }^{33} \mathrm{~J}$. R. Melcher, Continuum electromechanics / james $r$. melcher, Includes index. (MIT Press, Cambridge, Mass).

${ }^{34}$ H. A. Pohl, "Some effects of nonuniform fields on dielectrics", Journal of Applied Physics 29, 1182-1188 (1958).

${ }^{35}$ X. Wang, X.-B. Wang, and P. R. C. Gascoyne, "General expressions for dielectrophoretic force and electrorotational torque derived using the maxwell stress tensor method", Journal of Electrostatics 39, 277-295 (1997).

${ }^{36}$ David J. Griffiths, Introduction to electrodynamics, in collab. with Internet Archive (Prentice Hall, 1999), 602 pp.

${ }^{37}$ E. Schmidt, Droplet_electro-bounce: project files for droplet electro-bounce, original-date: 2017-06-06T22:20:50Z, (June 18, 2017) https : / / github . com/7deeptide/Droplet_electro-bounce.

${ }^{38}$ G. M. Sessler, ed., Electrets, 2nd ed., Topics in Applied Physics (SpringerVerlag, Berlin Heidelberg, 1987).

${ }^{39}$ O. Heaviside, Electrical papers, Vol. 2 (Cambridge University Press, 2011).

${ }^{40} \mathrm{~T}$. Wu, Y. Suzuki, and N. Kasagi, "Low-voltage droplet manipulation using liquid dielectrophoresis on electret", Journal of Micromechanics and Microengineering 20, 085043 (2010). 
${ }^{41} \mathrm{~J}$. Van Turnhout, Thermally stimulated discharge of polymer electrets: a study on nonisothermal dielectric relaxation phenomena (Elsevier Science \& Technology, 1975).

${ }^{42}$ Y. Wada, H. Oguchi, M. Hara, H. Asanuma, and H. Kuwano, "Stacking electrets for electrostatic vibration energy harvesters", in Digest tech. PowerMEMS 2012 workshop (2012), pp. 504-507.

${ }^{43} \mathrm{H}$. Ni, R. C. Amme, and Y. Jin, "Desalination by electret technology", Desalination 174, 237-245 (2005).

${ }^{44}$ C. G. L. Furmidge, "Studies at phase interfaces. i. the sliding of liquid drops on solid surfaces and a theory for spray retention", Journal of Colloid Science 17, 309-324 (1962).

${ }^{45}$ D. K. Davies, "The examination of the electrical properties of insulators by surface charge measurement", Journal of Scientific Instruments 44, 521 (1967).

${ }^{46}$ M. M. Perlman, Electrets, charge storage, and transport in dielectrics, Vol. 2 (Dielectrics and Insulation Division, Electrochemical Society, 1973).

${ }^{47}$ H. T. M. Haenen, "The characteristic decay with time of surface charges on dielectrics", Journal of Electrostatics 1, 173-185 (1975).

${ }^{48}$ G. F. L. Ferreira and M. T. Figueiredo, "Corona charging of electrets: models and results", IEEE Transactions on Electrical Insulation 27, 719738 (1992).

${ }^{49}$ J. Schindelin, I. Arganda-Carreras, E. Frise, V. Kaynig, M. Longair, T. Pietzsch, S. Preibisch, C. Rueden, S. Saalfeld, B. Schmid, J.-Y. Tinevez, D. J. White, V. Hartenstein, K. Eliceiri, P. Tomancak, and A. Cardona, "Fiji: an open-source platform for biological-image analysis", Nature Methods 9, 676-682 (2012).

${ }^{50}$ C. A. Schneider, W. S. Rasband, and K. W. Eliceiri, "NIH image to ImageJ: 25 years of image analysis", Nature Methods 9, 671-675 (2012).

${ }^{51} \mathrm{~K}$. Li, The image stabilizer plugin for ImageJ (Feb. 2008).

${ }^{52}$ T. E. Oliphant, "Python for scientific computing", Computing in Science Engineering 9, 10-20 (2007).

${ }^{53}$ A. Savitzky and M. J. E. Golay, "Smoothing and differentiation of data by simplified least squares procedures.", Analytical Chemistry 36, 1627-1639 (1964).

${ }^{54} \mathrm{R}$. W. Schafer, "What is a savitzky-golay filter? [lecture notes]", IEEE Signal Processing Magazine 28, 111-117 (2011). 
${ }^{55}$ A. C. Hindmarsh, "ODEPACK, a systematized collection of ODE solvers, RS stepleman et al.(eds.), north-holland, amsterdam,(vol. 1 of), pp. 5564.", IMACS transactions on scientific computation 1, 55-64 (1983).

${ }^{56}$ J. Nocedal and S. Wright, Numerical optimization, 2nd ed., Springer Series in Operations Research and Financial Engineering (Springer-Verlag, New York, 2006).

${ }^{57}$ G. A. Wood, "Data smoothing and differentiation procedures in biomechanics", Exercise and Sport Sciences Reviews 10, 308 (1982).

${ }^{58}$ T. Kolda, R. Lewis, and V. Torczon, "Optimization by direct search: new perspectives on some classical and modern methods", SIAM Review 45, $385-482$ (2003).

${ }^{59}$ J. A. Nelder and R. Mead, "A simplex method for function minimization", The Computer Journal 7, 308-313 (1965).

${ }^{60}$ C. J. Price, I. D. Coope, and D. Byatt, "A convergent variant of the nelder-mead algorithm", Journal of Optimization Theory and Applications 113, 5-19 (2002).

${ }^{61}$ W. Spendley, G. R. Hext, and F. R. Himsworth, "Sequential application of simplex designs in optimisation and evolutionary operation", Technometrics 4, 441-461 (1962).

${ }^{62} \mathrm{P} . \mathrm{R}$. Bevington, Data reduction and error analysis for the physical sciences (1969)

${ }^{63}$ K. Jaqaman and G. Danuser, "Linking data to models: data regression", Nature Reviews Molecular Cell Biology 7, 813 (2006).

${ }^{64}$ T. Takamatsu, Y. Hashimoto, M. Yamaguchi, and T. Katayama, "Theoretical and experimental studies of charged drop formation in a uniform electric field", Journal of Chemical Engineering of Japan 14, 178-182 (1981).

${ }^{65}$ S. Schiaffino and A. A. Sonin, "Molten droplet deposition and solidification at low weber numbers", Physics of Fluids 9, 3172-3187 (1997).

${ }^{66}$ J. Moláček and J. W. M. Bush, "A quasi-static model of drop impact", Physics of Fluids 24, 127103 (2012).

${ }^{67}$ A. Gopinath and D. L. Koch, "Collision and rebound of small droplets in an incompressible continuum gas", Journal of Fluid Mechanics 454, 145201 (2002).

${ }^{68}$ K. Okumura, F. Chevy, D. Richard, D. Quéré, and C. Clanet, "Water spring: a model for bouncing drops", EPL (Europhysics Letters) 62, 237 (2003). 
${ }^{69}$ D. Duvivier, R. Rioboo, M. Voue, and J. D. Coninck, "Drop impact on superhydrophobic surfaces - varying gravitational effects", Atomization and Sprays 22 (2012) 10.1615/AtomizSpr . 2012005704.

${ }^{70}$ D. Bartolo, F. Bouamrirene, É. Verneuil, A. Buguin, P. Silberzan, and S. Moulinet, "Bouncing or sticky droplets: impalement transitions on superhydrophobic micropatterned surfaces", EPL (Europhysics Letters) 74, 299 (2006).

${ }^{71}$ M. Reyssat, A. Pépin, F. Marty, Y. Chen, and D. Quéré, "Bouncing transitions on microtextured materials", EPL (Europhysics Letters) 74, 306 (2006). 